\title{
THE LOW-LUMINOSITY END OF THE RADIUS-LUMINOSITY RELATIONSHIP FOR ACTIVE GALACTIC NUCLEI
}

\author{
Misty C. Bentz ${ }^{1}$, Kelly D. Denney ${ }^{2,16}$, Catherine J. Grier ${ }^{3}$, Aaron J. Barth ${ }^{4}$, Bradley M. Peterson ${ }^{3,5}$, \\ Marianne Vestergaard ${ }^{2,6}$, Vardha N. Bennert ${ }^{7}$, Gabriela Canalizo $^{8}$, Gisella De Rosa ${ }^{3}$, Alexei V. Filippenko ${ }^{9}$, \\ Elinor L. Gates ${ }^{10}$, Jenny E. Greene ${ }^{11}$, Weidong Li ${ }^{9,17}$, Matthew A. Malkan ${ }^{12}$, Richard W. Pogge ${ }^{3,5}$, Daniel Stern ${ }^{13}$, \\ Tommaso Treu ${ }^{14}$, AND JoNG-HaK WoO ${ }^{15}$ \\ ${ }^{1}$ Department of Physics and Astronomy, Georgia State University, Atlanta, GA 30303, USA; bentz@ chara.gsu.edu \\ ${ }^{2}$ Dark Cosmology Center, Niels Bohr Institute, Juliane Maries Vej 30, DK-2100 Copenhagen Ø, Denmark \\ ${ }^{3}$ Department of Astronomy, The Ohio State University, 140 West 18th Avenue, Columbus, OH 43210, USA \\ ${ }^{4}$ Department of Physics and Astronomy, 4129 Frederick Reines Hall, University of California, Irvine, CA 92697, USA \\ ${ }^{5}$ Center for Cosmology and AstroParticle Physics, The Ohio State University, 191 West Woodruff Avenue, Columbus, OH 43210, USA \\ ${ }^{6}$ Steward Observatory, University of Arizona, 933 N Cherry Avenue, Tucson, AZ 85721, USA \\ ${ }^{7}$ Physics Department, California Polytechnic State University, San Luis Obispo, CA 93407, USA \\ ${ }^{8}$ Department of Physics and Astronomy, University of California, Riverside, CA 92521, USA \\ ${ }^{9}$ Department of Astronomy, University of California, Berkeley, CA 94720, USA \\ ${ }^{10}$ University of California Observatories/Lick Observatory, P.O. Box 85, Mount Hamilton, CA 95140, USA \\ ${ }^{11}$ Department of Astrophysical Sciences, Princeton University, Peyton Hall - Ivy Lane, Princeton, NJ 08544, USA \\ 12 Department of Physics and Astronomy, University of California, Los Angeles, CA 90095, USA \\ 13 Jet Propulsion Laboratory, California Institute of Technology, 4800 Oak Grove Drive, Pasadena, CA 91109, USA \\ ${ }^{14}$ Department of Physics, University of California, Santa Barbara, CA 93106, USA \\ ${ }^{15}$ Astronomy Program, Department of Physics and Astronomy, Seoul National University, Seoul, Republic of Korea \\ Received 2012 December 19; accepted 2013 March 6; published 2013 April 5
}

\begin{abstract}
We present an updated and revised analysis of the relationship between the $\mathrm{H} \beta$ broad-line region (BLR) radius and the luminosity of the active galactic nucleus (AGN). Specifically, we have carried out two-dimensional surface brightness decompositions of the host galaxies of nine new AGNs imaged with the Hubble Space Telescope Wide Field Camera 3. The surface brightness decompositions allow us to create "AGN-free" images of the galaxies, from which we measure the starlight contribution to the optical luminosity measured through the ground-based spectroscopic aperture. We also incorporate 20 new reverberation-mapping measurements of the $\mathrm{H} \beta$ time lag, which is assumed to yield the average $\mathrm{H} \beta$ BLR radius. The final sample includes 41 AGNs covering four orders of magnitude in luminosity. The additions and updates incorporated here primarily affect the low-luminosity end of the $R_{\mathrm{BLR}}-L$ relationship. The best fit to the relationship using a Bayesian analysis finds a slope of $\alpha=0.533_{-0.033}^{+0.035}$, consistent with previous work and with simple photoionization arguments. Only two AGNs appear to be outliers from the relationship, but both of them have monitoring light curves that raise doubt regarding the accuracy of their reported time lags. The scatter around the relationship is found to be $0.19 \pm 0.02$ dex, but would be decreased to 0.13 dex by the removal of these two suspect measurements. A large fraction of the remaining scatter in the relationship is likely due to the inaccurate distances to the AGN host galaxies. Our results help support the possibility that the $R_{\mathrm{BLR}}-L$ relationship could potentially be used to turn the BLRs of AGNs into standardizable candles. This would allow the cosmological expansion of the universe to be probed by a separate population of objects, and over a larger range of redshifts.
\end{abstract}

Key words: galaxies: active - galaxies: nuclei - galaxies: photometry - galaxies: Seyfert

\section{INTRODUCTION}

The ability to determine black hole masses is a crucial step toward understanding the link between galaxies and their black holes, as well as the details of the black hole environment. To date, dynamical methods have resulted in measurements of some 50 black hole masses. However, these methods require that the gravitational influence of the black hole on the stars or gas be spatially resolved, effectively limiting the reach of current dynamical methods to galaxies no further than $\sim 150 \mathrm{Mpc}$ for even the most massive black holes (see Gültekin et al. 2009).

Active galactic nuclei (AGNs), in contrast, are some of the most luminous objects in the universe, and are thus capable of providing us with the leverage needed to probe the growth and

\footnotetext{
${ }^{16}$ Marie Curie Fellow.

${ }^{17}$ Deceased 2011 December 12.
}

evolution of black holes at any significant cosmological distance. However, AGNs are also so rare that even the nearest are generally too distant for current instruments to spatially resolve the radius of influence of the black hole and provide a local calibration for their masses. Instead, the most successful technique for measuring black hole masses in AGNs is reverberation mapping (Blandford \& McKee 1982). Reverberation mapping requires high-quality spectrophotometric monitoring of an AGN over an extended period of time. The line-emitting regions that give rise to the characteristic AGN spectral signatures are photoionized by the hot accretion disk around the black hole. The continuum flux (which arises from the accretion disk or very close to it) varies with time, and these variations are echoed later by changes in the flux of the broad emission lines. The delay time between the continuum variations and the broad-line variations can be measured by cross correlation of the light curves and gives the light-travel time across the broadline region (BLR), or the radius of the BLR when multiplied by 
the speed of light. In effect, reverberation mapping substitutes high temporal resolution for high spatial resolution, allowing us to probe regions of gas that are only $\sim 0.01 \mathrm{pc}$ in extent (comparable to the inner region of the Oort Cloud in our own solar system; Brown et al. 2004) in the centers of arbitrarily distant galaxies. Combining the BLR radius with the mean velocity of the BLR gas, as measured from the Doppler broadening of the emission lines, and assumptions or indirect estimates of the virial coefficient gives a direct constraint on the black hole mass via the virial theorem.

The validity of reverberation masses has been upheld by several independent lines of evidence. A subset of objects in the reverberation sample have measurements for several different emission lines throughout the ultraviolet and optical portions of their spectra, and the multiple emission lines show a virial behavior (e.g., Peterson \& Wandel 1999, 2000; Kollatschny 2003; Bentz et al. 2010). Two AGNs in the current reverberation sample-NGC 3227 and NGC 4151 - are sufficiently close that dynamical modeling has successfully determined their black hole masses, and both the stellar dynamical masses (Davies et al. 2006; Onken et al. 2007) and gas dynamical masses (Hicks \& Malkan 2008) agree with the reverberation-based masses within the uncertainties. Furthermore, a fully general Bayesian modeling code has recently been developed to analyze reverberation-mapping data sets and place limits on the black hole mass and the BLR geometry and dynamics (Pancoast et al. 2011). When applied to the reverberation-mapping data for Arp 151 (Brewer et al. 2011) and Mrk 50 (Pancoast et al. 2012), the method recovers a black hole mass that is essentially the same value as that determined from the reverberation method outlined above, for standard assumptions of the virial coefficient (Bentz et al. 2009b; Barth et al. 2011).

Reverberation mapping has yielded black hole masses for $\sim 50$ AGNs thus far (Peterson et al. 2004; Bentz et al. 2009b). The BLR radius-luminosity correlation $\left(R_{\mathrm{BLR}} \propto L^{\alpha}\right)$ derived from this reverberation sample is the basis for all secondary techniques used to estimate black hole masses in distant AGNs (e.g., Laor 1998; Wandel et al. 1999; McLure \& Jarvis 2002; Vestergaard \& Peterson 2006). The power of the $R_{\mathrm{BLR}}-L$ relationship comes from the simplicity of using it to quickly estimate $M_{\mathrm{BH}}$ for large samples of objects, even at high redshift, with only a single spectrum per object.

This simplicity has led to the $R_{\mathrm{BLR}}-L$ relationship being heavily utilized in the literature. A small sampling of studies that have utilized the $R_{\mathrm{BLR}}-L$ relationship in the last few years includes investigations of $M_{\mathrm{BH}}$ in the most distant quasars (e.g., Willott et al. 2010; Mortlock et al. 2011), black hole mass functions and Eddington ratio distributions through cosmic history (e.g., Greene \& Ho 2007b; Vestergaard et al. 2008; Vestergaard \& Osmer 2009; Kelly et al. 2009; Schulze \& Wisotzki 2010), cosmic evolution of black holes and their host galaxies (e.g., Woo et al. 2008; Merloni et al. 2010; Bennert et al. 2010), the nature of narrow-line Seyfert 1 galaxies (e.g., Mathur et al. 2012; Papadakis et al. 2010), duty cycles of quasars (e.g., Shankar et al. 2009), accretion properties of various types of AGNs (e.g., Wang et al. 2009; Cao 2010; Trump et al. 2011), studies of relativistic jets and the jet-disk connection (e.g., Sambruna et al. 2006; Tavecchio et al. 2007; Ghisellini \& Tavecchio 2010), studies of black holes in dwarf and low-mass galaxies (e.g., Greene \& Ho 2007a; Dong et al. 2007; Thornton et al. 2008), and studies of optical transients (e.g., Drake et al. 2011).

Because of the utility of the $R_{\mathrm{BLR}}-L$ relationship, much work has gone into removing biases and noise from the reverberation database. Previous determinations of the $R_{\mathrm{BLR}}-L$ relationship used luminosity measurements from ground-based spectra and found the slope to be $\alpha \approx 0.7$ (Kaspi et al. 2000, 2005). To achieve the low level of uncertainties in the flux calibration necessary for reverberation mapping, a large (e.g., $5^{\prime \prime} \times 7^{\prime \prime}$ ) spectroscopic aperture is typically employed. Therefore, for all the nearby objects with reverberation masses, a substantial fraction of the observed luminosity is actually the result of the host-galaxy starlight and not the AGN itself. The entire lowluminosity end of the $R_{\mathrm{BLR}}-L$ relationship was overestimated in $L$, in effect, artificially steepening the slope.

Bentz et al. (2006b) analyzed Hubble Space Telescope (HST) Advanced Camera for Surveys (ACS) images of the nearest reverberation-mapped AGNs and their host galaxies taken through the F550M medium-band $V$ filter. The flux contribution of starlight through the ground-based spectroscopic monitoring aperture was measured from each image, and the reverberationmapping luminosities were corrected accordingly. The resultant $R_{\mathrm{BLR}}-L$ relationship was found, as expected, to have a much flatter slope $(\alpha=0.52 \pm 0.04$ compared to $\alpha=0.67 \pm$ $0.05)$, consistent with simple photoionization expectations. Consequently, all of the remaining objects in the reverberationmapped sample were imaged with ACS in a similar manner with the intent of properly accounting for the starlight in each object, even when that contribution was assumed to be small. While the slope of the relationship did not change much $(\alpha=0.52 \pm 0.06$; Bentz et al. 2009a), the scatter in the relationship was reduced from $\sim 40 \%$ to $\sim 35 \%$. The scarcity of measurements anchoring the low-luminosity end, in particular, then became apparent.

In the meantime, much effort has gone into replacing noisy and poorly sampled reverberation data sets and increasing the overall range of BLR radii probed. The past several years in particular have seen a huge amount of effort invested in reverberation-mapping experiments that preferentially target AGNs with relatively low luminosities. The Lick AGN Monitoring Project (LAMP) campaign targeted low-luminosity AGNs to more fully populate the low-luminosity end of the $R_{\mathrm{BLR}}-L$ relationship and succeeded in measuring $\mathrm{H} \beta$ BLR radii for eight new AGNs (Bentz et al. 2009b). Multiple recent campaigns at MDM Observatory have mainly focused on replacing poorly sampled or noisy reverberation data sets with high signal-tonoise ratio $(\mathrm{S} / \mathrm{N})$, high temporal cadence spectroscopy (Denney et al. 2010; Grier et al. 2012) to allow better constraints on the BLR structure and kinematics.

Given the number of updates, improvements, and additions to the reverberation database, we undertook a full recalibration of the $R_{\mathrm{BLR}}-L$ relationship in an effort to provide a more accurately calibrated relationship for the community to use when estimating black hole masses in AGNs. In particular, our new calibration is more accurate at the low-luminosity end where $L_{*}$ and sub- $L_{*}$ galaxies tend to reside.

We assume a standard $\Lambda \mathrm{CDM}$ cosmology of $H_{0}=$ $72 \mathrm{~km} \mathrm{~s}^{-1} \mathrm{Mpc}^{-1}, \Omega_{\mathrm{M}}=0.3, \Omega_{\Lambda}=0.7$ throughout this work.

\section{NEW H $\beta$ BLR MEASUREMENTS}

Recent reverberation-mapping campaigns have focused mainly on the low-luminosity end of the $R_{\mathrm{BLR}}-L$ relationship and provide several new $\mathrm{H} \beta$ BLR measurements to the reverberation sample. The measurements come in three separate flavors: (1) replacement measurements for targets of previous reverberation campaigns for which the light curves were noisy or undersampled and led to poor or biased determinations of the $\mathrm{H} \beta$ BLR radius, (2) additional measurements for targets of previous 
reverberation campaigns that already have accurate $\mathrm{H} \beta$ time-lag measurements, and (3) H $\beta$ BLR measurements for new objects that have not been previously examined with reverberation mapping. We provide a brief summary of each of the monitoring programs with the new results that we incorporate here. The interested reader should refer to the original manuscripts reporting the $\mathrm{H} \beta$ BLR measurements for more details.

MDM 2005. 3C 390.3 was the subject of a 2005 monitoring campaign at MDM Observatory that resulted in an additional $\mathrm{H} \beta$ radius and luminosity measurement for this object (Dietrich et al. 2012).

MDM 2007. Denney et al. (2010) describe the results of a 2007 monitoring program at MDM and other observatories that had a goal of obtaining high-quality, densely sampled light curves to search for velocity-resolved time lags in the emission lines. $\mathrm{H} \beta$ BLR measurements were derived for six AGNs through this program, three of which were replacements for poor-quality data sets, and two of which were additional measurements for AGNs with other reliable measurements. The final object, Mrk 290, was new. Mrk 290 was also included in the 2008 LAMP sample of AGNs (see below) but did not exhibit strong variations during that campaign. The inclusion of Mrk 290 in the LAMP sample led to it being included in the HST Cycle 17 imaging campaign that we describe below, from which we are able to derive the starlight correction to the luminosity.

LAMP 2008. The 2008 LAMP campaign targeted AGNs with estimated black hole masses in the range $10^{6}-10^{7} \mathrm{M}_{\odot}$. Measurements of the $\mathrm{H} \beta$ BLR radius were determined for eight new objects, and an additional measurement of the $\mathrm{H} \beta$ BLR radius in the well-studied AGN NGC 5548 was also determined (Bentz et al. 2009b). NGC 5548 was the only galaxy in the LAMP sample with the appropriate HST imaging to allow a host-galaxy starlight correction. In the next sections we detail the HST Cycle 17 imaging program through which we obtained the necessary images for the remainder of the LAMP sample, the host-galaxy surface brightness modeling of those images, and the derived starlight corrections to the ground-based spectroscopic monochromatic luminosities at $5100 \AA$.

MDM 2010. Additional measurements of $\mathrm{H} \beta$ radii were determined for four AGNs in the reverberation sample during a 2010-2011 campaign at MDM and other observatories (Grier et al. 2012; B. M. Peterson et al., in preparation). Two other AGNs, Mrk 6 and Mrk 1501, were new targets and reliable $\mathrm{H} \beta$ radii were determined for them. Unfortunately, there is no suitable HST imaging from which to measure the starlight correction to the spectroscopic luminosity. We are therefore unable to include them in this analysis of the $R_{\mathrm{BLR}}-L$ relationship.

The addition of nine new AGNs to the reverberation sample along with 11 replacement or additional data sets for previously monitored AGNs allows us to revisit the calibration of the $R_{\mathrm{BLR}}-L$ relationship, and in particular to examine the form of the relationship at the lower luminosity end. We include in this analysis all reverberation data sets for which (1) there is a reliable time lag measured for the $\mathrm{H} \beta$ emission line, and (2) there is medium $V$-band (F547M or F550M) HST imaging available so the host-galaxy contribution to the rest-frame $5100 \AA$ flux can be determined and removed. Other archival HST images are available for some of the objects not included here, but these images are not suitable for our analysis for one of three reasons: (1) they were taken with a different filter and therefore include emission lines from the galaxy and/or the narrowline region, both of which would have to be corrected, and they would require assumptions about the unknown underlying stellar populations in the galaxy, and/or (2) the exposures are too shallow to accurately constrain the host-galaxy surface brightness profiles, or (3) the images are heavily saturated in the nucleus, with strong bleeding and a loss of information at the galaxy center. We do not include reverberation measurements of other Balmer lines in this analysis because previous work (Kaspi et al. 2000; Bentz et al. 2009b) has shown that there are differences in the mean time lags determined for different Balmer lines, most likely caused by radiative-transfer effects in the BLR clouds. We are left with the sample of 41 AGNs that are listed in Table 1.

\section{HST OBSERVATIONS AND DATA REDUCTION}

Nine AGNs with new $\mathrm{H} \beta$ time lags were imaged during Cycle 17 (GO-11662, PI: Bentz) with the Wide Field Camera 3 (WFC3) UVIS channel through the F547M (Strömgren $y$ ) filter $\left(\lambda_{c}=5447 \AA\right.$ and $\left.\Delta \lambda=650 \AA\right)$. This imaging setup allowed us to probe the continuum flux from the AGN and the host galaxy while avoiding strong emission lines. One orbit was dedicated to each object, and each orbit was divided into two sets of exposures separated by a dithering maneuver to improve the sampling of the WFC3 point-spread function (PSF) and facilitate in the rejection of cosmic rays and detector artifacts (such as transient warm pixels). To maximize the dynamic range of the final images, each set of three exposures was graduated in time, with exposure times of approximately $30 \mathrm{~s}, 300 \mathrm{~s}$, and $690 \mathrm{~s}$. We did not dither during an exposure sequence to ensure that all three images were taken at the same position. Most of our targets were compact enough to fit on a single chip of the UVIS channel, but for NGC 6814 we employed a larger dithering maneuver to ensure that there was no loss of information because of the gap between the chips. Details of the HST observations are given in Table 2 .

We were able to correct for saturation in the long exposures by making use of the linear nature of charge-coupled devices. Saturated pixels in the nucleus of each galaxy were identified in each image by consulting the data quality frames from the HST pipeline. These saturated pixels were clipped from the image and replaced by the same pixels from a shallower, unsaturated exposure, but scaled up by the exposure-time ratio. Cosmic rays were cleaned with the Laplacian cosmic ray identification package L. A. Cosmic (van Dokkum 2001). All of the frames for a single object were then combined with the multidrizzle task to create a distortion-free image of each AGN host galaxy. The final combined, drizzled images are shown in Figure 1 with the ground-based spectroscopic monitoring apertures overlaid. It can easily be seen that the host galaxy of each AGN contributes a significant amount of light within the monitoring aperture.

\section{GALAXY SURFACE BRIGHTNESS DECOMPOSITIONS}

An important component of calibrating the $R_{\mathrm{BLR}}-L$ relationship is properly correcting the $L$ measurements for the contribution from host-galaxy starlight. The method we employed here is similar to that described by Bentz et al. (2006b, 2009a), where the analysis of 32 galaxies in our sample is reported; it relies on using the software program GALFIT (Peng et al. 2002) to model the surface brightness profiles of the host-galaxy images. GALFIT is a nonlinear least-squares two-dimensional image-fitting algorithm. We used the latest version of GALFIT (Version 3) which allows for the modeling of spiral arms, rings, and irregular shapes (see Peng et al. 2010 for a full description and various examples). 
Table 1

Object List

\begin{tabular}{|c|c|c|c|c|c|c|}
\hline Object & $\begin{array}{c}\alpha_{\mathrm{J} 2000} \\
(\mathrm{hr} \text { min sec) }\end{array}$ & $\begin{array}{l}\delta_{\mathrm{J} 2000} \\
\left({ }^{\circ}{ }^{\prime \prime \prime}\right)\end{array}$ & $z$ & $\begin{array}{c}D_{\mathrm{L}}^{\mathrm{a}} \\
(\mathrm{Mpc})\end{array}$ & $\begin{array}{c}A_{B} \mathrm{~b} \\
(\mathrm{mag})\end{array}$ & $\begin{array}{l}\text { Alternate } \\
\text { Name }\end{array}$ \\
\hline Mrk 335 & 000619.5 & +201210 & 0.02579 & 113 & 0.129 & PG 0003+199 \\
\hline PG 0026+129 & 002913.6 & +131603 & 0.14200 & 672 & 0.258 & \\
\hline PG $0052+251$ & 005452.1 & +252538 & 0.15500 & 740 & 0.172 & \\
\hline Fairall 9 & 012345.8 & -584821 & 0.04702 & 209 & 0.097 & \\
\hline Mrk 590 & 021433.6 & -004600 & 0.02639 & 115 & 0.136 & NGC 863 \\
\hline $3 \mathrm{C} 120$ & 043311.1 & +052116 & 0.03301 & 145 & 1.078 & Mrk 1506 \\
\hline Ark 120 & 051611.4 & -000859 & 0.03271 & 142 & 0.466 & Mrk 1095 \\
\hline Mrk 79 & 074232.8 & +494835 & 0.02219 & 97 & 0.257 & \\
\hline PG 0804+761 & 081058.6 & +760242 & 0.10000 & 461 & 0.126 & \\
\hline Mrk 110 & 092512.9 & +521711 & 0.03529 & 155 & 0.047 & \\
\hline PG $0953+414$ & 095652.4 & +411522 & 0.23410 & 1172 & 0.046 & \\
\hline NGC 3227 & 102330.6 & +195154 & 0.00386 & $23.5 \pm 2.4$ & 0.082 & \\
\hline Mrk 142 & 102531.3 & +514035 & 0.04494 & 199 & 0.058 & \\
\hline NGC 3516 & 110647.5 & +723407 & 0.00884 & 38 & 0.154 & \\
\hline SBS $1116+583 \mathrm{~A}$ & 111857.7 & +580324 & 0.02787 & 122 & 0.042 & \\
\hline Arp 151 & 112536.2 & +542257 & 0.02109 & 92 & 0.050 & Mrk 40 \\
\hline NGC 3783 & 113901.7 & -374419 & 0.00973 & $25.1 \pm 5.0$ & 0.432 & \\
\hline Mrk 1310 & 120114.3 & -034041 & 0.01956 & 85 & 0.112 & \\
\hline NGC 4051 & 120309.6 & +443153 & 0.00234 & $17.1 \pm 3.4$ & 0.047 & \\
\hline NGC 4151 & 121032.6 & +392421 & 0.00332 & $16.6 \pm 3.3$ & 0.100 & \\
\hline Mrk 202 & 121755.0 & +583935 & 0.02102 & 92 & 0.073 & \\
\hline NGC 4253 & 121826.5 & +294846 & 0.01293 & 56 & 0.071 & Mrk 766 \\
\hline PG $1226+023$ & 122906.7 & +020309 & 0.15834 & 758 & 0.075 & $3 C 273$ \\
\hline PG 1229+204 & 123203.6 & +200929 & 0.06301 & 283 & 0.098 & Mrk $771 \&$ Ton 1542 \\
\hline NGC 4593 & 123939.4 & -052039 & 0.00900 & $37.3 \pm 7.5$ & 0.089 & Mrk 1330 \\
\hline NGC 4748 & 125212.4 & -132453 & 0.01463 & 63 & 0.187 & \\
\hline PG 1307+085 & 130947.0 & +081949 & 0.15500 & 739 & 0.122 & \\
\hline Mrk 279 & 135303.4 & +691830 & 0.03045 & 134 & 0.058 & \\
\hline PG $1411+442$ & 141348.3 & +440014 & 0.08960 & 410 & 0.031 & \\
\hline NGC 5548 & 141759.5 & +250812 & 0.01718 & 75 & 0.074 & \\
\hline PG 1426+015 & 142906.6 & +011706 & 0.08647 & 394 & 0.115 & \\
\hline Mrk 817 & 143622.1 & +584739 & 0.03146 & 138 & 0.024 & PG $1434+590$ \\
\hline Mrk 290 & 153552.3 & +575409 & 0.02958 & 130 & 0.055 & \\
\hline PG $1613+658$ & 161357.2 & +654310 & 0.12900 & 606 & 0.096 & Mrk 876 \\
\hline PG $1617+175$ & 162011.3 & +172428 & 0.11244 & 522 & 0.151 & Mrk 877 \\
\hline PG $1700+518$ & 170124.8 & +514920 & 0.29200 & 1510 & 0.127 & \\
\hline 3C 390.3 & 184209.0 & +794617 & 0.05610 & 251 & 0.259 & \\
\hline NGC 6814 & 194240.6 & -101925 & 0.00521 & 22 & 0.664 & \\
\hline Mrk 509 & 204409.7 & -104325 & 0.03440 & 151 & 0.208 & \\
\hline PG 2130+099 & 213227.8 & +100819 & 0.06298 & 283 & 0.161 & II Zw 136 \& Mrk 1513 \\
\hline NGC 7469 & 230315.6 & +085226 & 0.01632 & 71 & 0.250 & Mrk 1514 \\
\hline
\end{tabular}

Notes.

a Distances were estimated from the redshifts of the AGNs except for five cases: NGC 3227, NGC 3783, NGC 4051, NGC 4151, and NGC 4593. The distances and uncertainties for the last four of these objects are from an average of distance moduli to neighboring galaxies in the Tully et al. (2008) study of the local velocity anomaly, with distance uncertainties estimated at 20\%. The distance to NGC 3227 is based on the SBF method (Tonry et al. 2001) for NGC 3226, with which NGC 3227 is currently interacting. Additional details are given in the text.

b Values are from the Schlafly \& Finkbeiner (2011) recalibration of the Schlegel et al. (1998) dust map.

For the surface brightness decomposition of each of the nine new HST host-galaxy images in this work, we employed a tilted plane for the background sky flux and a TinyTim (Krist 1993) model for the unresolved AGN. TinyTim models were generated for each specific AGN by creating a model at the specific detector position of each of the pointings and combining these models through multidrizzle in the same way that the AGN images were combined. Host-galaxy bulges, disks, and bars were all fit with Sérsic (1968) profiles of the basic form

$$
\Sigma(r)=\Sigma_{e} \exp \left[-\kappa\left(\left(\frac{r}{r_{e}}\right)^{1 / n}-1\right)\right],
$$

where $\Sigma_{e}$ is the pixel surface brightness at the effective radius $r_{e}$. The Sérsic index, $n$, has a value of 1 for an exponential disk, 4 for a de Vaucouleurs (1948) profile, and 0.5 for a Gaussian. Bulge and bar components were modeled by allowing the Sérsic index to vary with no constraints while disks were modeled by holding the Sérsic index fixed at a value of 1 . Fits that resulted in bulge Sérsic indices outside of the range $\sim 0.1-6$ were considered unphysical and therefore unacceptable. In these cases, we required multiple PSF models in the center of the galaxy, offset by fractions of a pixel, to keep the Sérsic index of the bulge from running up the maximum value allowed by Galfit, $n=20$. A high Sérsic index has a very peaky shape with strong wings, and can mimic a PSF+sky model. 

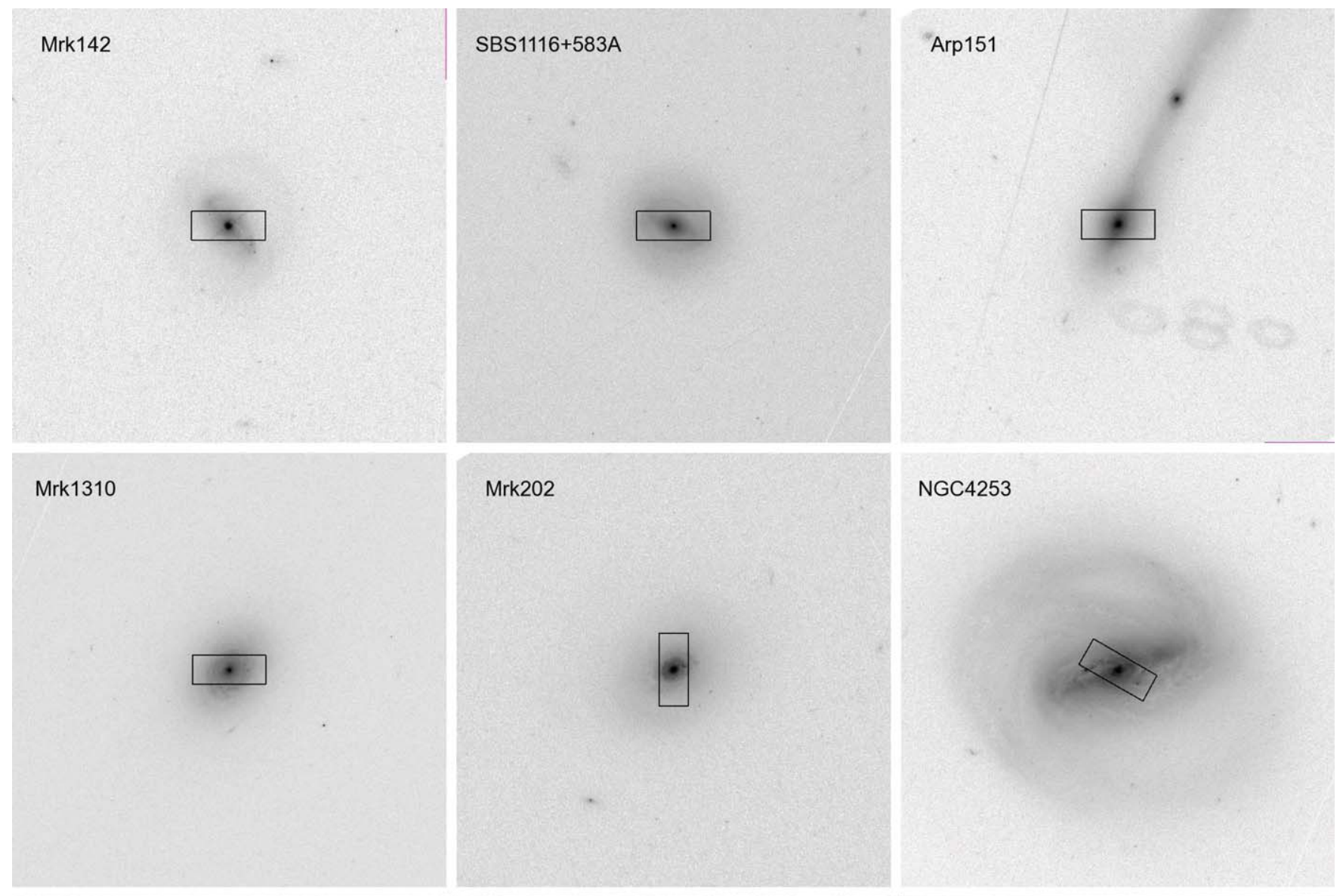

NGC4253
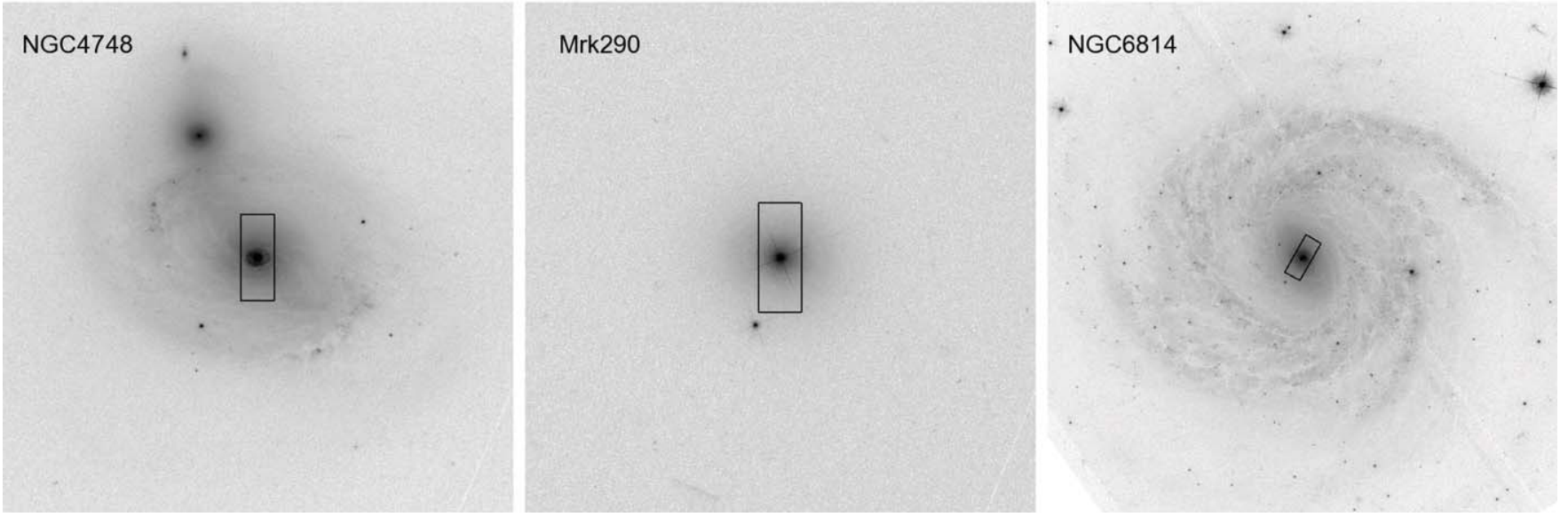

Figure 1. HST WFC3 F547M images of the AGN host galaxies, displayed with an inverted logarithmic stretch. The black rectangles show the geometry and orientation of each ground-based spectroscopic monitoring aperture. The size of the region displayed is $1^{\prime} \times 1^{\prime}$, except for NGC 6814 which is displayed in a $2^{\prime} \times 2^{\prime}$ box. For all images, north is up and east is to the left.

Table 2

HST Observation Log

\begin{tabular}{lccc}
\hline \hline Object & $\begin{array}{c}\text { Date Observed } \\
(\text { yyyy-mm-dd) }\end{array}$ & $\begin{array}{c}\text { Total Exposure } \\
\text { Time }(\mathrm{s})\end{array}$ & $\begin{array}{c}\text { Roll Angle } \\
\left({ }^{\circ} \mathrm{E} \text { of N) }\right.\end{array}$ \\
\hline Mrk 142 & $2010-04-22$ & 2450 & -8.29 \\
SBS 1116+583A & $2010-06-06$ & 2510 & -28.1 \\
Arp 151 & $2010-04-09$ & 2450 & 29.7 \\
Mrk 1310 & $2009-12-02$ & 2240 & 157.1 \\
Mrk 202 & $2010-04-14$ & 2510 & 25.5 \\
Mrk 766 & $2010-06-21$ & 2270 & -22.5 \\
NGC 4748 & $2010-06-28$ & 2250 & -24.1 \\
Mrk 290 & $2010-07-25$ & 2520 & -22.0 \\
NGC 6814 & $2010-05-06$ & 2240 & 120.7 \\
\hline
\end{tabular}

A runaway Sérsic index in our galaxy fitting is likely because of the well-known PSF mismatch that can occur between TinyTim models and WFC3 images due to spacecraft "breathing" and/or jitter, but could potentially be caused by any marginally resolved nuclear flux from hot gas or star clusters. We assume here that the cause is PSF mismatch and ascribe all of the flux in these multiple PSF models (which we assume are modeling a single physical component) to the AGN itself, and we describe various tests of the validity of this assumption below.

GALFIT allows for surface brightness decompositions that can be as simple or complicated as the user may wish. The ultimate goal of the surface brightness modeling in this project was to accurately remove the AGN PSF, thereby creating an 

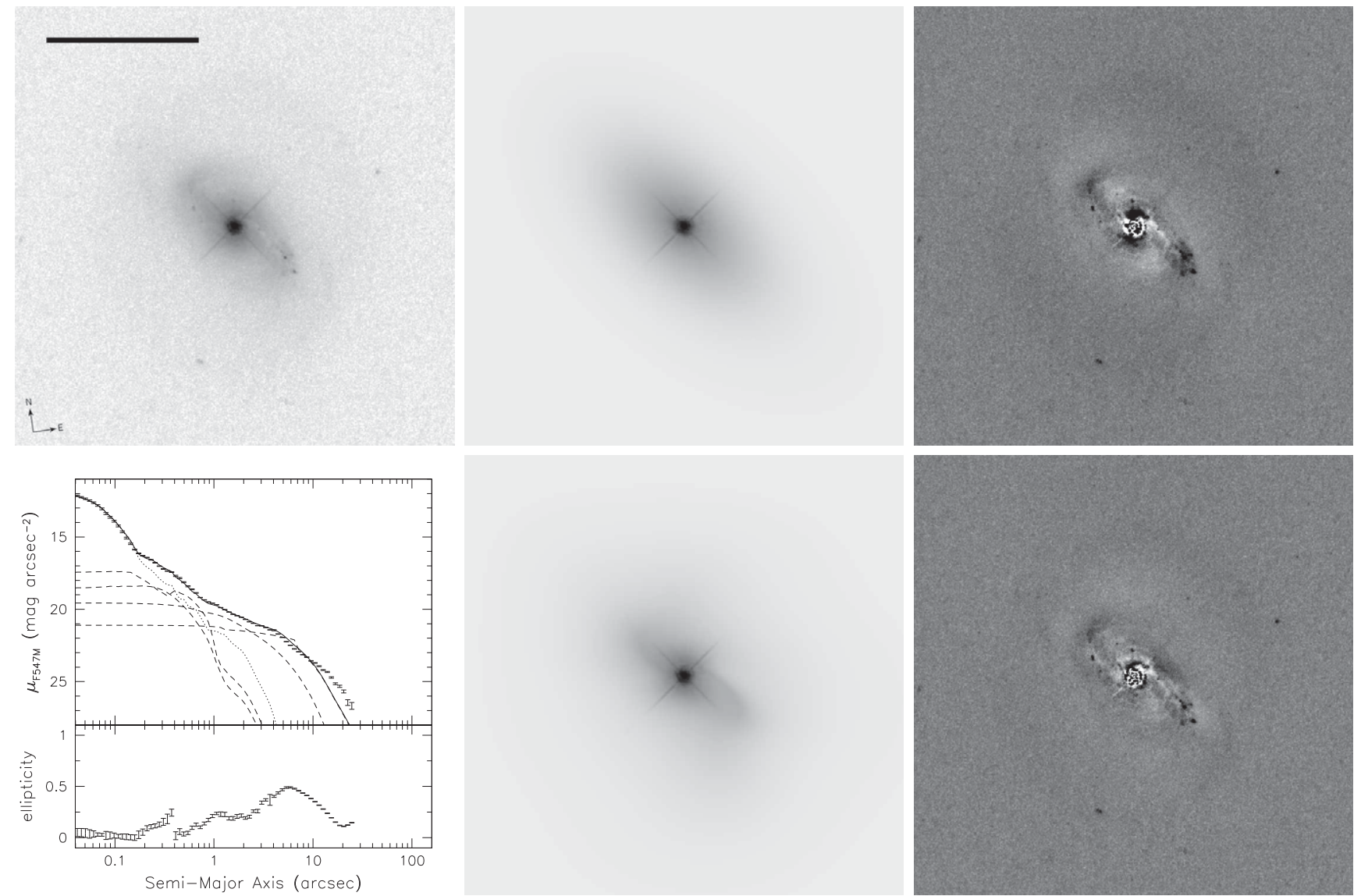

Figure 2. Image (top left), models (middle), and residuals (right) for Mrk 142. The upper panels display the "simple" models and their residuals, and the bottom panels display the "optimal" models and their residuals. The scale of the black bar in the top left panel is 10 arcsec and the compass in the bottom corner of the panel shows the directions north and east. The galaxy image and models are displayed with an inverted logarithmic stretch, and the residuals are displayed with an inverted linear stretch centered around zero counts. The bottom left panel shows the one-dimensional surface brightness of the galaxy (data points), the best-fit model (solid line), and each of the individual best-fit model components (PSF = dotted line, all others = dashed lines), with the ellipticity of the galaxy displayed below.

"AGN-free" image of each host galaxy from which the starlight contribution could be measured. Bentz et al. (2009a) assume uncertainties of $0.1 \mathrm{mag}$ in the measured host-galaxy flux based in the range of acceptable models that could be found to fit an image. Here, we investigate the uncertainty in the best-fit models by carrying out two independent sets of surface brightness decompositions for each of the host-galaxy images in this study.

The first set of models, which we will refer to as the "optimal" models, include multiple surface brightness components and make use of power-law rotation to model spiral arms, Fourier modes to account for "bending" of the ellipse modeling the light distribution and other asymmetric flux distributions, and truncation functions to allow for the modeling of rings. These models, which are shown in the bottom panels of Figures 2-10, are the best representations for the actual two-dimensional surface brightness distributions of the host galaxies.

The second set of models, which we refer to as "simple" models, do not make use of power-law rotations, Fourier modes, or truncation functions. The simple models typically require a factor of three fewer free parameters than the optimal models and they are computationally much faster to run and to converge, but they less accurately represent the two-dimensional surface brightness profiles of the AGN host galaxies, as can be seen in the top panels of Figures 2-10. Conversely, the optimal models do a good job of reproducing the relative flux in each pixel in the images, but the importance of this, other than being aesthetically pleasing, is not clear and the physical interpretation of each model component is not straightforward to determine.

Tables 3-11 give the parameters determined for the "optimal" and "simple" fits for each of the nine galaxies fit here. The formats of the tables are as follows: Column 1 gives the note for the type of fit described ("optimal" or "simple"); Column 2 gives the component number of the fit, generally in order of increasing angular size and increasing angular offset from the center of the galaxy; and Column 3 gives the description for the type of model component (or components in the case of a PSF model and tilted plane sky model). The remaining columns describe the various parameters of each model, with Column 11 listing any notes relevant to the models. We give a brief description of the remaining columns below, but the interested reader is referred to Peng et al. (2010) for further details of the models and their parameters employed by GALFIT.

For the PSF models, Columns 4 and 5 are the angular offsets in arcseconds from the center of the galaxy (defined as the location of the AGN PSF) in the $x$ and $y$ directions, respectively. Column 6 is the integrated magnitude of the PSF model. For the sky models, Column 8 gives the average value of the sky background level in counts at the geometric center of the image, and Columns 9 and 10 give the flux gradients in the $x$ and $y$ directions, respectively.

Sérsic models are listed with Columns 4 and 5 as the angular offsets in arcseconds from the center of the galaxy in the $x$ and $y$ directions, respectively. Column 6 gives the integrated 

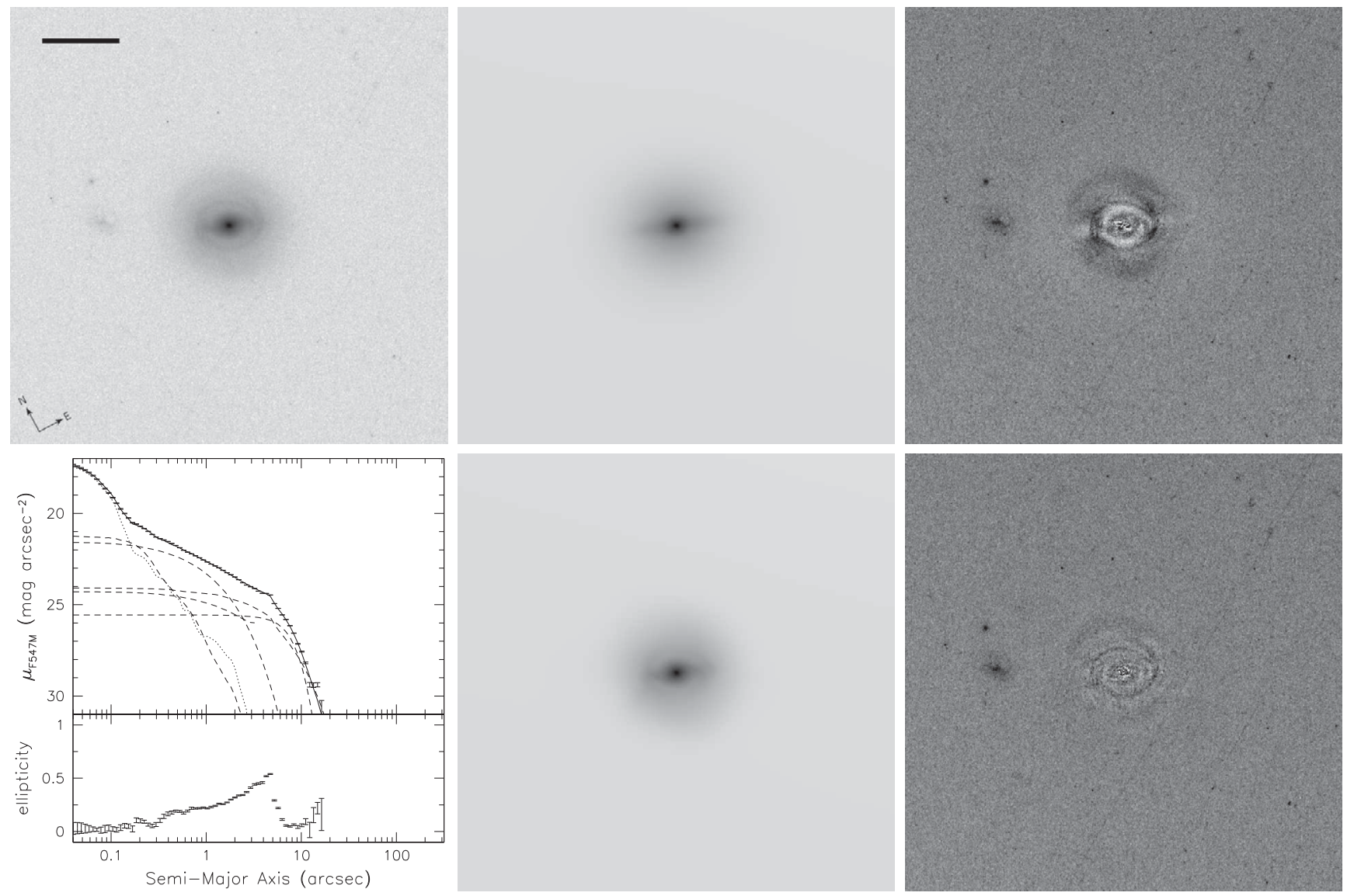

Figure 3. Same as Figure 2, but for SBS 1116+583A.
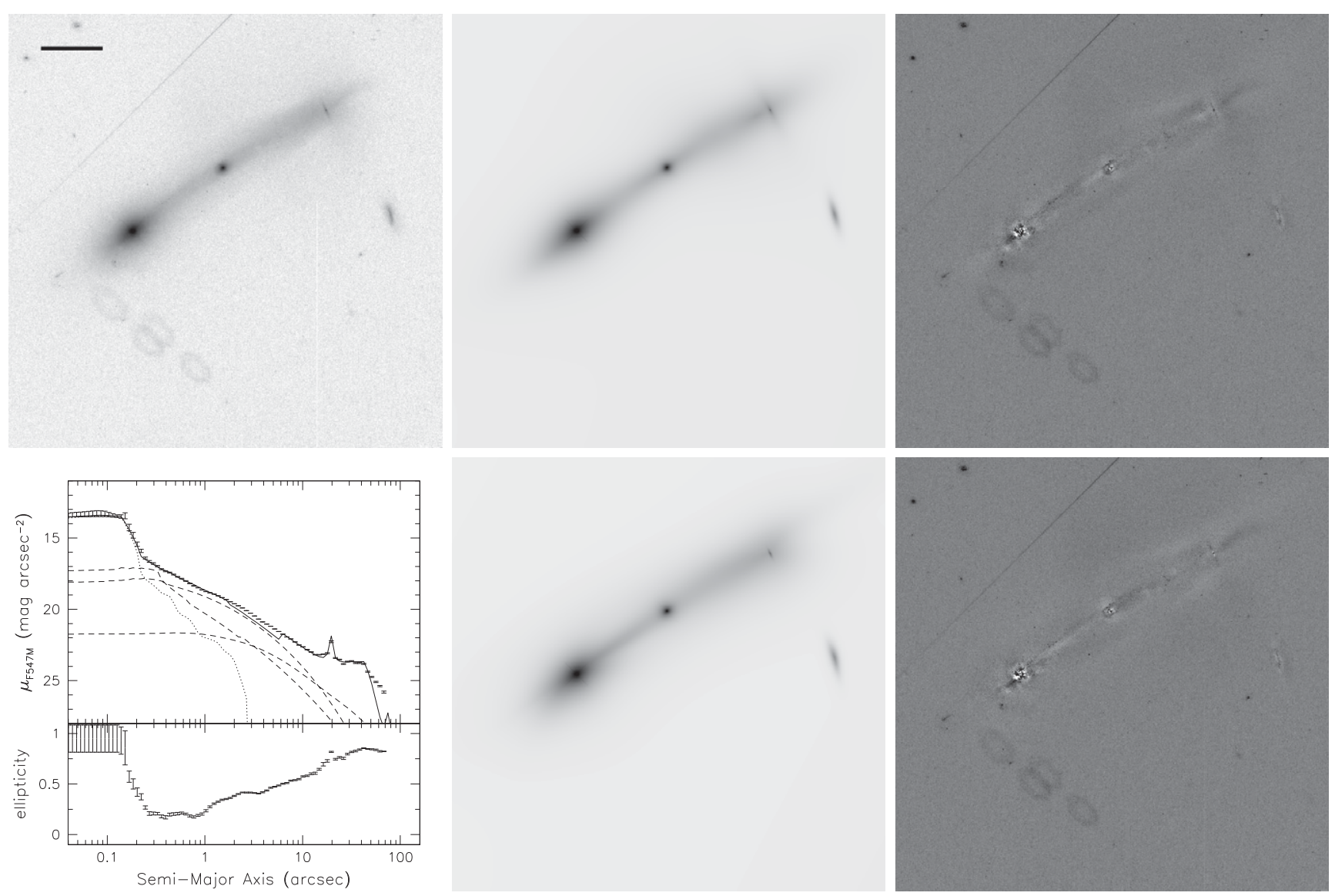

Figure 4. Same as Figure 2, but for Arp 151. The "O" shapes in the images are reflections in the optics from a nearby bright object. 

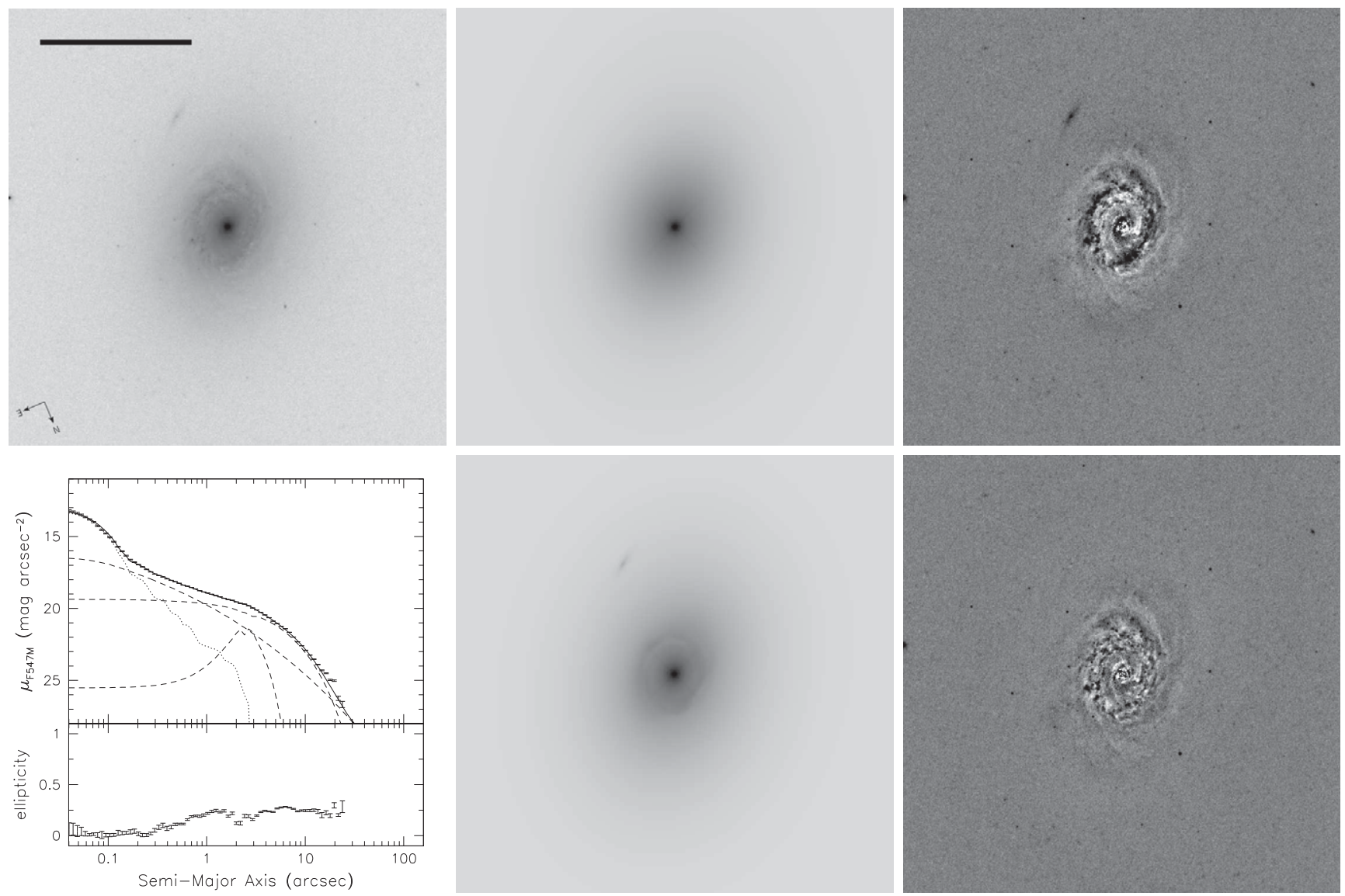

Figure 5. Same as Figure 2, but for Mrk 1310
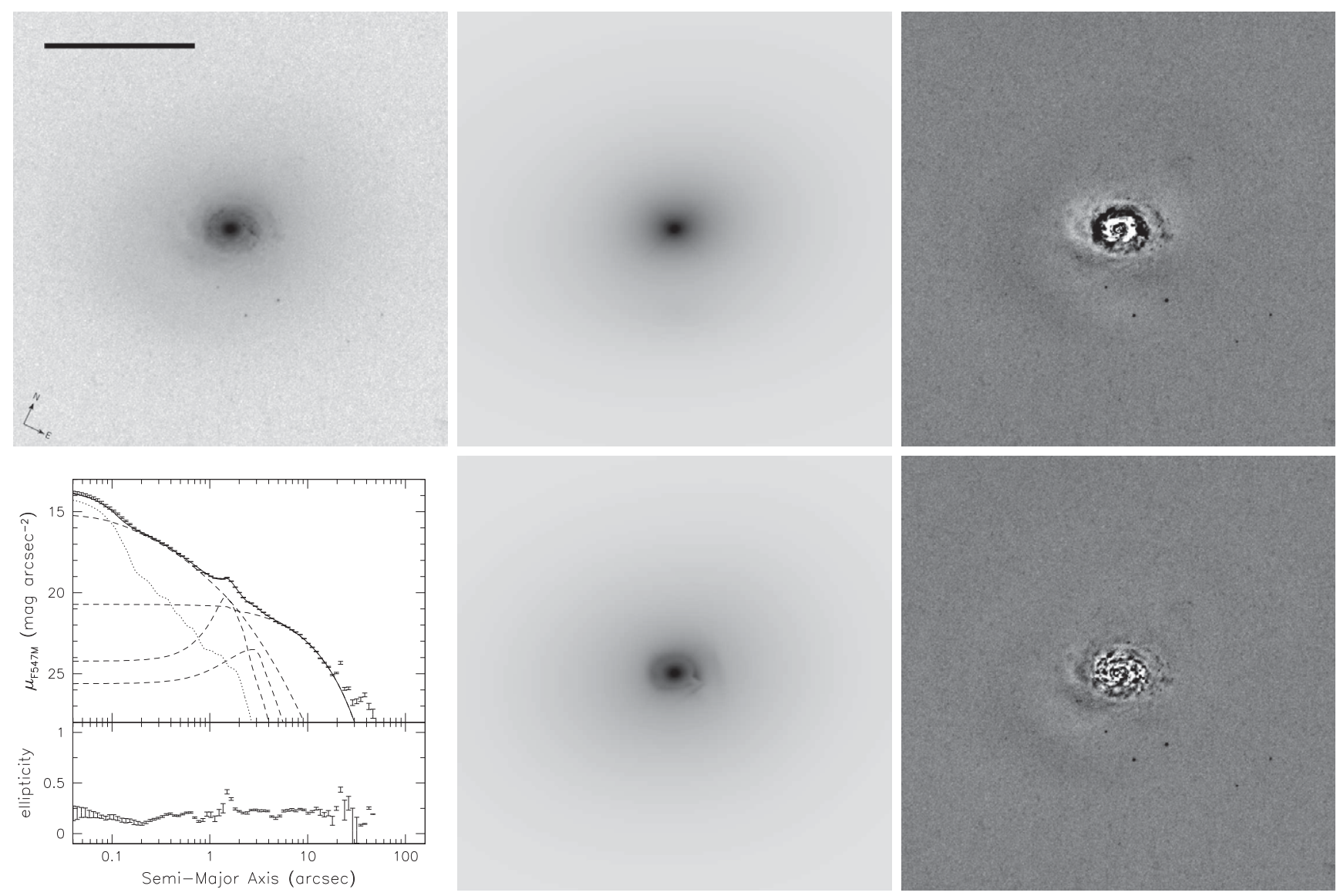

Figure 6. Same as Figure 2, but for Mrk 202. 

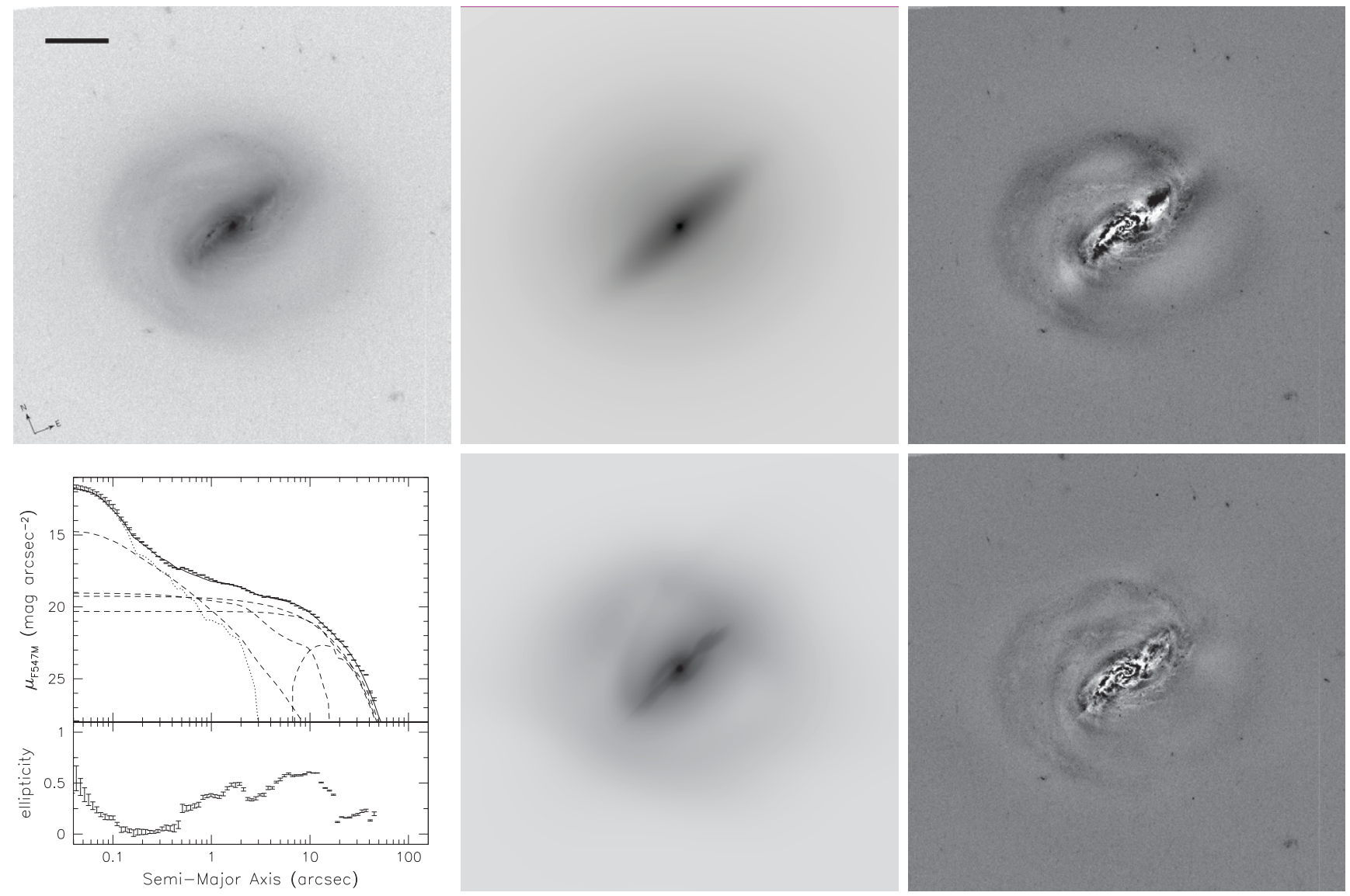

Figure 7. Same as Figure 2, but for NGC 4253 (Mrk 766).
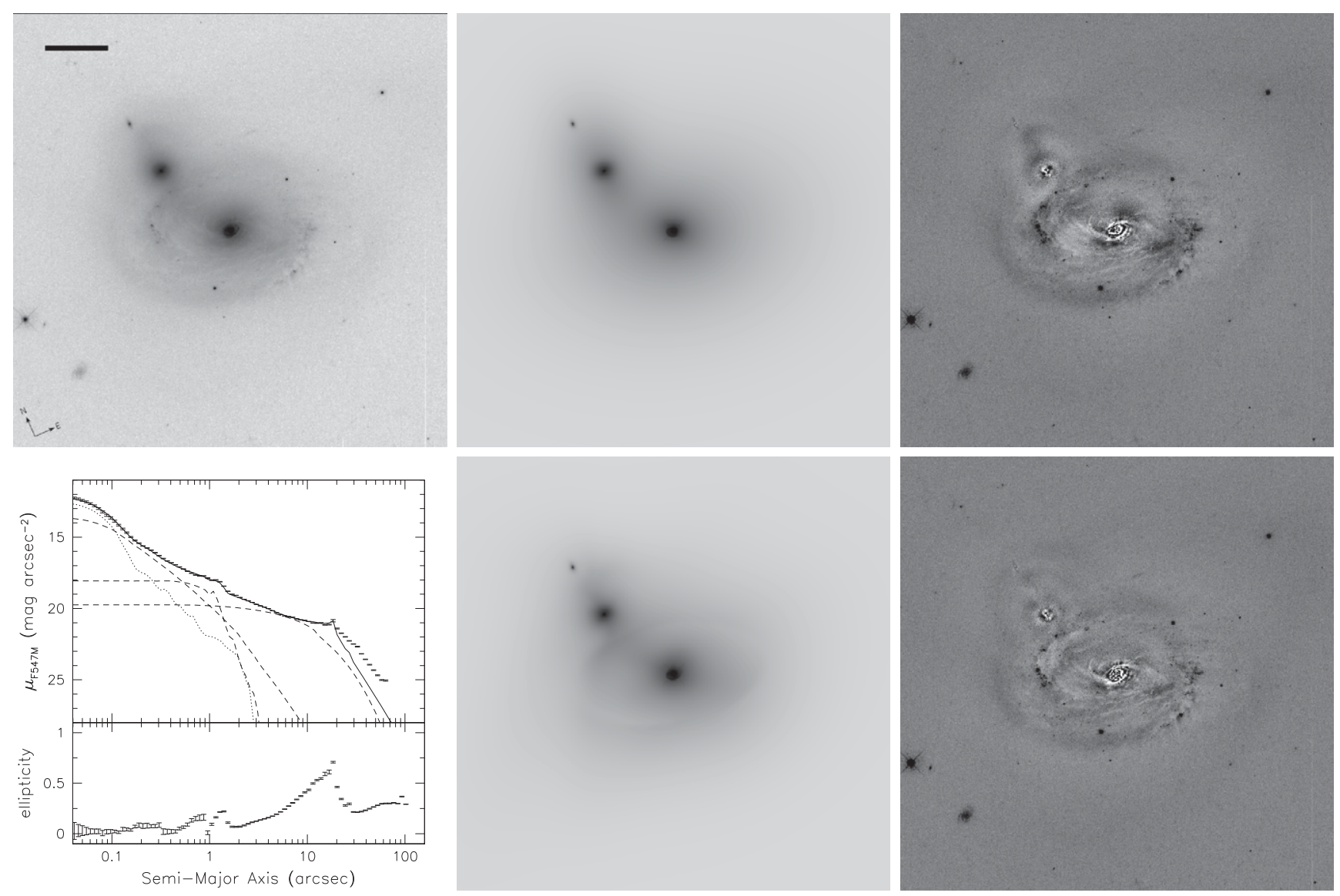

Figure 8. Same as Figure 2, but for NGC 4748. 

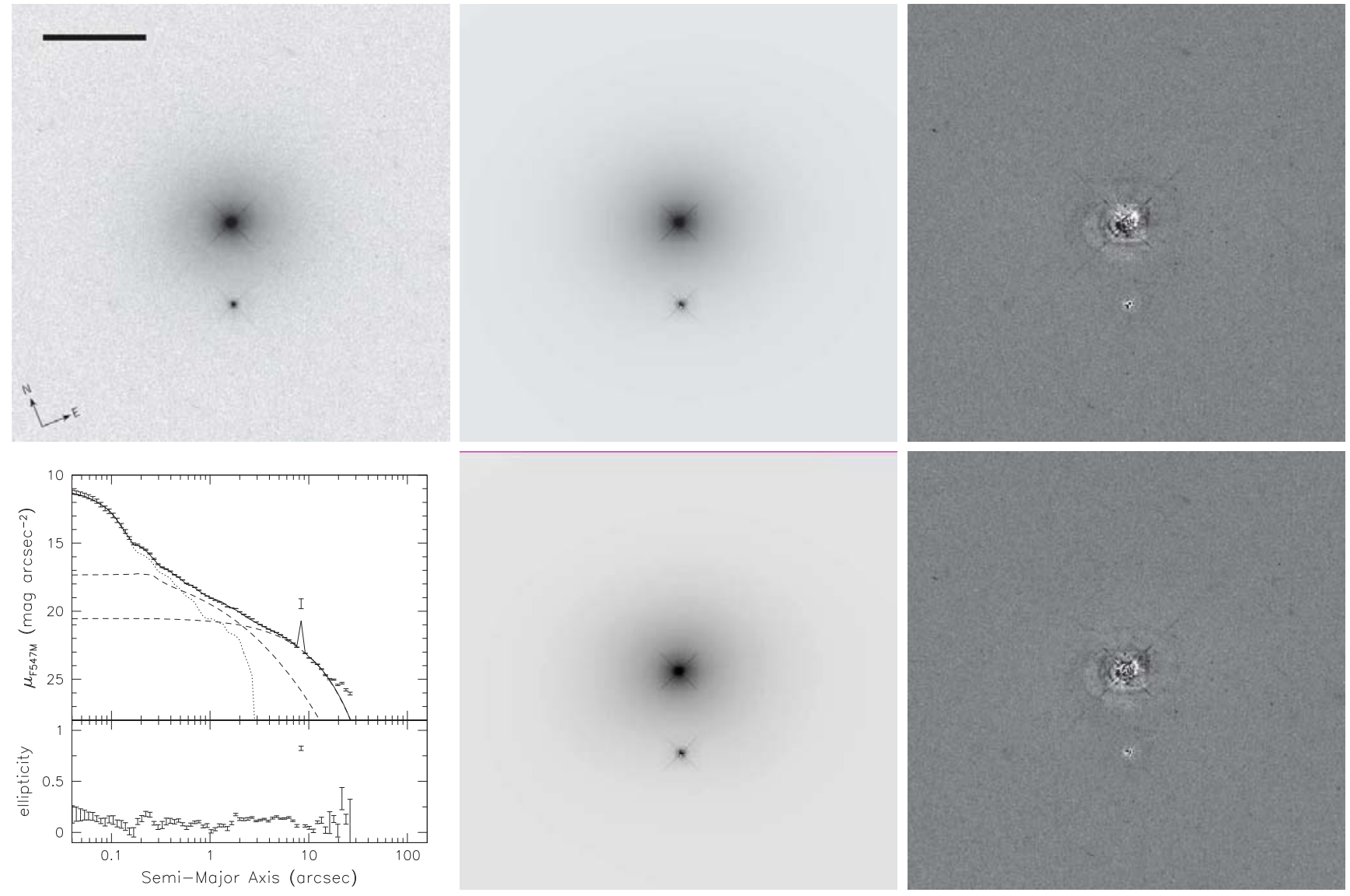

Figure 9. Same as Figure 2, but for Mrk 290. The small jump in surface brightness at $9^{\prime \prime}$ is due to the bright field star in the image.
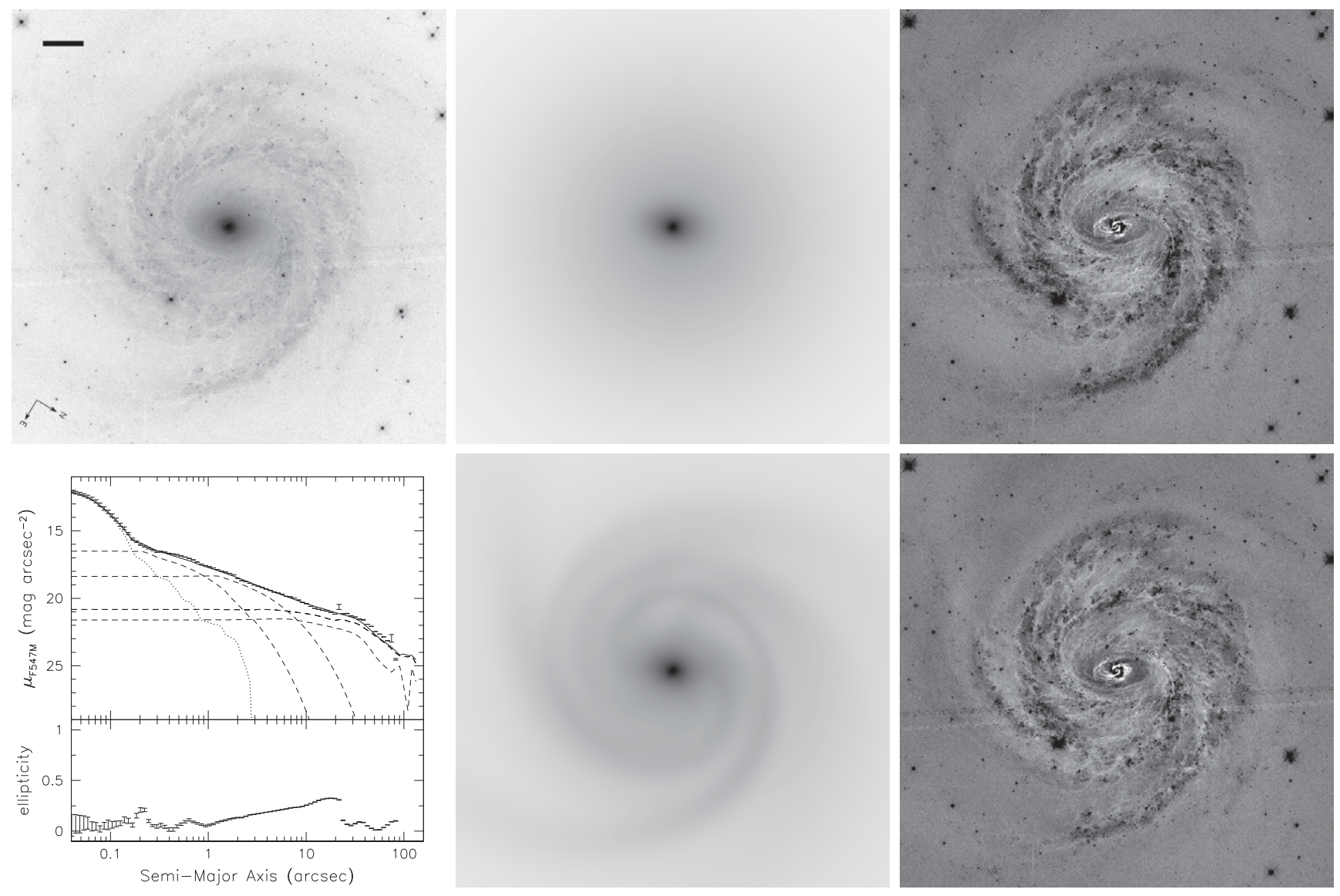

Figure 10. Same as Figure 2, but for NGC 6814. 
Table 3

Surface Brightness Decomposition-Mrk 142

\begin{tabular}{|c|c|c|c|c|c|c|c|c|c|c|}
\hline \multirow[t]{2}{*}{ Fit } & & \multirow{3}{*}{$\begin{array}{c}\text { PSF+sky } \\
\text { sersic } \\
\text { power } \\
\text { fourier } \\
\text { (3) }\end{array}$} & \multirow{3}{*}{$\begin{array}{c}\Delta x\left(^{\prime \prime}\right) \\
\Delta x\left(^{\prime \prime}\right) \\
\ldots \\
\ldots \\
(4)\end{array}$} & $\begin{array}{l}\Delta y\left(^{(\prime)}\right) \\
\Delta y\left(^{\prime \prime}\right) \\
r_{\text {in }}\left({ }^{\prime \prime}\right)\end{array}$ & \multirow{2}{*}{$\begin{array}{l}m_{\text {stmag }}{ }^{\text {a }} \\
m_{\text {stmag }} \\
r_{\text {out }}\left(^{\prime \prime}\right) \\
(\text { deg })\end{array}$} & $\begin{array}{c}\cdots \\
r_{\mathrm{e}}\left({ }^{\prime \prime}\right) \\
\theta_{\mathrm{rot}}(\mathrm{deg})\end{array}$ & \multirow[t]{2}{*}{$\begin{array}{c}\text { Sky (counts) } \\
\qquad \begin{array}{c}\alpha \\
\alpha \\
\phi(\operatorname{deg})\end{array}\end{array}$} & \multirow{2}{*}{\multicolumn{2}{|c|}{$\begin{array}{cc}\frac{d \text { sky }}{d x}\left(10^{-4} \text { counts }\right) & \frac{d \text { sky }}{d y}\left(10^{-4} \text { counts }\right) \\
b / a & \text { P.A. }(\mathrm{deg}) \\
\theta_{\text {incl }}(\text { deg }) & \theta_{\text {sky }}(\mathrm{deg}) \\
\text { mode: } a_{\mathrm{m}}, \phi(\mathrm{deg})\end{array}$}} & \multirow{3}{*}{$\begin{array}{l}\text { Note } \\
\\
\text { (11) }\end{array}$} \\
\hline & & & & mode: $a_{\mathrm{m}}, \phi(\mathrm{deg})$ & & mode: $a_{\mathrm{m}}, \phi(\mathrm{deg})$ & & & & \\
\hline (1) & (2) & & & (5) & (6) & $(7)$ & (8) & $(9)$ & $(10)$ & \\
\hline \multirow[t]{17}{*}{ Optimal } & 1,2 & PSF+sky & 0 & 0 & 16.10 & $\ldots$ & 33.2 & 6.1 & -7.5 & \\
\hline & 3 & sersic & 0.12 & -0.10 & 18.78 & 0.17 & {$[1.0]$} & 0.12 & 118.7 & \\
\hline & & power & $\ldots$ & 0.22 & 0.38 & -94.5 & 0.4367 & 61.6 & 2.1 & \\
\hline & & fourier & $\ldots$ & 1: 0.90 & -70.1 & $3: 0.22$ & 0.5 & $4:-0.038$ & -6.1 & \\
\hline & & fourier & $\ldots$ & 5: 0.028 & -1.1 & & & & & \\
\hline & 4 & sersic & 0.30 & 0.29 & 19.44 & 0.22 & 0.6 & 0.34 & 33.2 & \\
\hline & & fourier & $\ldots$ & $1:-0.31$ & 99.5 & 3: 0.193 & -2.9 & $4: 0.2468$ & 34.8 & \\
\hline & & fourier & $\ldots$ & 5: 0.12 & 28.3 & & & & & \\
\hline & 5 & sersic & 0.05 & -0.07 & 17.40 & 4.82 & [1.0] & 0.28 & 30.0 & \\
\hline & & power & $\ldots$ & 0.96 & 2.24 & -36.3 & -1.202 & 65.1 & 19.1 & \\
\hline & & fourier & $\ldots$ & 1: -0.092 & -96.0 & $3:-0.053$ & 7.9 & $4: 0.028$ & 23.6 & \\
\hline & & fourier & $\ldots$ & 5: 0.014 & 11.8 & & & & & \\
\hline & 6 & sersic & -0.28 & -0.13 & 16.47 & 14.79 & [1.0] & 0.39 & -45.4 & \\
\hline & & power & $\ldots$ & -1.11 & 7.68 & -299.6 & 0.0523 & 70.0 & 148.1 & \\
\hline & & fourier & $\ldots$ & 1: 0.035 & 6.8 & 3: -0.049 & -37.5 & $4: 0.0556$ & -24.8 & \\
\hline & & fourier & $\ldots$ & $5:-0.042$ & 2.2 & & & & & \\
\hline & & merit & & $=222239$ & & $N_{\text {dof }}=$ & 10869913 & $N_{\text {free }}=81$ & $\chi_{v}^{2}=2.045$ & \\
\hline \multirow[t]{5}{*}{ Simple } & 1,2 & PSF+sky & 0 & 0 & 16.13 & $\ldots$ & 33.3 & 6.1 & -7.0 & \\
\hline & 3 & sersic & 0.14 & 0.33 & 19.32 & 0.31 & 0.7 & 0.45 & 65.4 & bar? \\
\hline & 4 & sersic & 0.02 & -0.09 & 18.39 & 0.31 & 1.0 & 0.48 & 67.0 & bar? \\
\hline & 5 & sersic & 0.05 & -0.09 & 16.31 & 4.50 & [1.0] & 0.55 & 47.2 & disk \\
\hline & & merit & \multicolumn{3}{|c|}{$\chi^{2}=22739314.0$} & \multicolumn{2}{|c|}{$N_{\text {dof }}=10869968$} & $N_{\text {free }}=26$ & $\chi_{v}^{2}=2.092$ & \\
\hline
\end{tabular}

Notes. Values in square brackets were held fixed during the surface brightness model fitting.

a The STmag magnitude system is based on the absolute physical flux per unit wavelength.

magnitude of the Sérsic component and Column 7 lists the effective radius in arcseconds. Column 8 gives the Sérsic index, which was held fixed at a value of 1.0 for exponential-disk components. Columns 9 and 10 are the axis ratio and the position angle of the major axis in the image. Note that images were fit at the orientation obtained during the observation, and the position angles listed would need to be corrected for the roll angle of the spacecraft to determine their orientation relative to north.

For the "optimal" fits, the Sérsic models were modified by power-law rotation, Fourier modes, and/or radial truncation functions. In the case of the truncation functions, where portions of the underlying model are removed, the Sérsic profile is listed as "sersic3" and we report the surface brightness at the break radius $\left(\Sigma_{\mathrm{b}}\right)$ and the break radius $\left(r_{\mathrm{b}}\right)$ itself in Columns 6 and 7 , rather than the integrated magnitude and effective radius.

Power-law rotations of a Sérsic profile are denoted by "power" in Column 3. Columns 5 and 6 list the inner and outer radii of rotation in arcseconds. Column 7 gives the rotation angle between the inner and outer radii and Column 8 is the power-law slope, denoted as $\alpha$. Columns 9 and 10 are the lineof-sight inclination angle of the disk, $\theta_{\text {incl }}$ (with $\theta_{\text {incl }}=0$ being equivalent to face-on), and the position angle of the rotation in the plane of the sky, $\theta_{\text {sky }}$.

Fourier-mode modifications to Sérsic profiles are denoted by "Fourier" in Column 3. Beginning with Columns 5 and 6, and continuing through Column 10, are the modes (e.g., $m=1$ or $m=3$ ) and their amplitudes $a_{\mathrm{m}}$ and phase angles $(\phi)$, where the phase angle is the relative angle between the Fourier mode and the position angle of the major axis of the parent Sérsic profile. To avoid degeneracy with the axis-ratio parameter for the Sérsic profiles, we did not make use of the $m=2$ Fourier mode.
Truncation functions were generally used to model rings in the galaxies and are denoted as "radial" in Column 3. Both inner and outer truncations were used, with each denoted appropriately. Columns 4 and 5 give the angular offsets of the center of the truncation function from the center of the parent Sérsic profile in the $x$ and $y$ directions, respectively. Column 7 gives the break radius of the truncation function, defined to be the radius at which the truncation function has a value of $99 \%$ of the flux of the untruncated Sérsic model at that same radius. Column 8 lists the softening length, $\Delta r_{\text {soft }}$, where $r_{\text {break }} \pm \Delta r_{\text {soft }}$ ( + for outer truncations, - for inner truncations) gives the radius at which the flux drops to $1 \%$ of the untruncated Sérsic model flux. Columns 9 and 10 give the axis ratio and position angle of the truncation function.

Finally, the last row of each fit gives the figures of merit for that particular surface brightness decomposition: $\chi^{2}$; the number of degrees of freedom, $N_{\text {dof }}$; the number of free parameters in the models, $N_{\text {free }}$, and the reduced $\chi^{2}, \chi_{\nu}^{2}$.

In addition, to test the suitability of our choice of using multiple TinyTim PSFs offset by fractions of a pixel to better model the AGN PSF in several objects, we carried out surface brightness decompositions using a variety of different PSF models. These included a very high $\mathrm{S} / \mathrm{N}$ WFC3 image of the white dwarf EGGR 102, a bright field star from the image of Mrk 290 (one of our targets), a fainter star in the field around EGGR 102, and Moffat (1969) fits to each of these stars. We also investigated the effect of convolving the image and the PSF model with a narrow Gaussian to ensure Nyquist sampling (e.g., Kim et al. 2008). In each of these tests, we only allowed a single component to model the AGN PSF and we compare the results of the test to the results obtained using the fitting procedures 
Table 4

Surface Brightness Decomposition-SBS 1116+583A

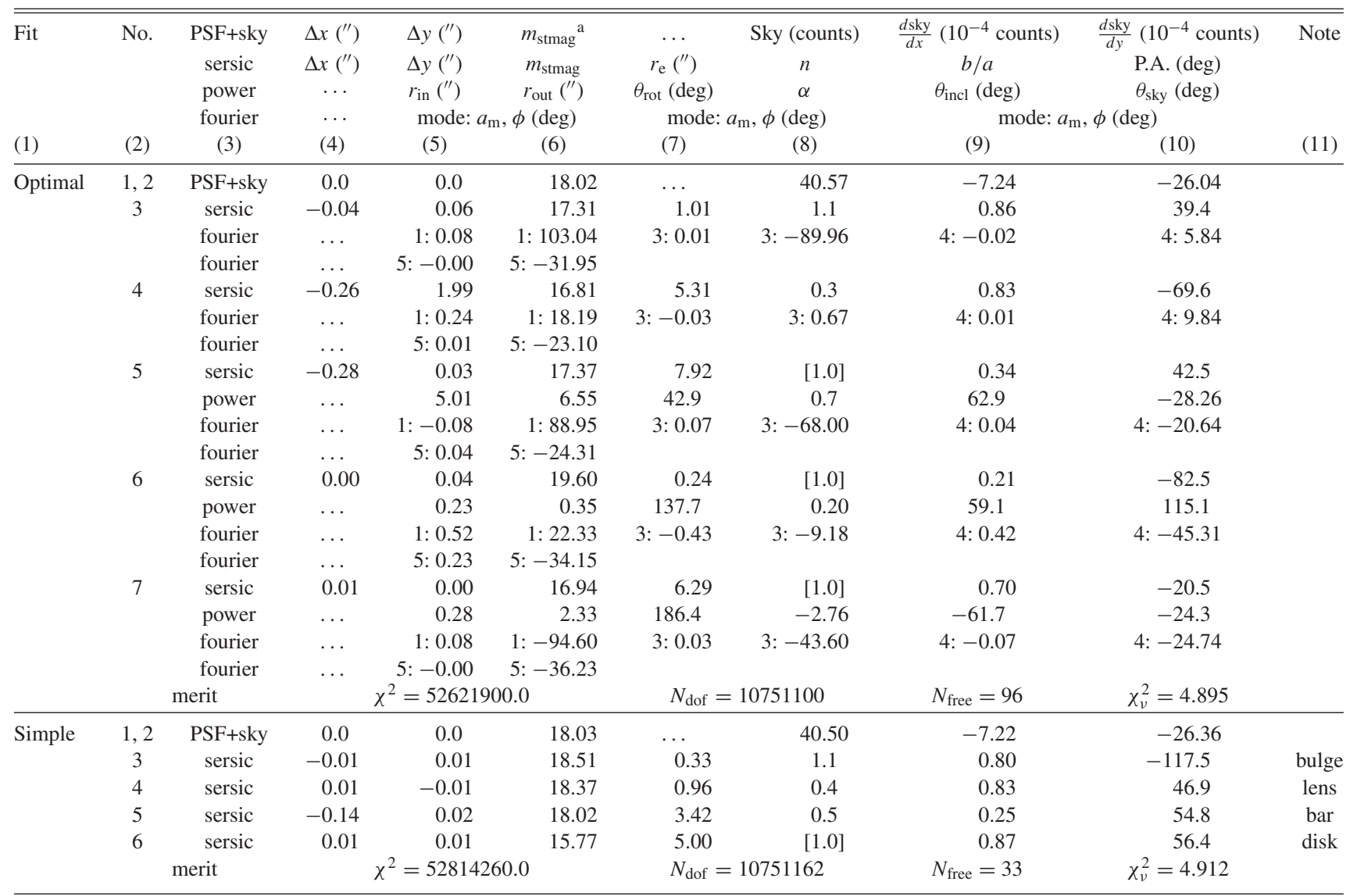

Notes. Values in square brackets were held fixed during the surface brightness model fitting.

a The STmag magnitude system is based on the absolute physical flux per unit wavelength.

described above and tabulated in Tables 3-11. When the image of a star was used as the PSF model, the difference in central host-galaxy flux measured from an "AGN-free" image was only $\sim 1 \%$, and it was only slightly higher $(\sim 2 \%)$ when a Moffat fit to a star image was used as the PSF model. Broadening the image and the PSF model caused the largest difference in central hostgalaxy flux, about $7 \%$, so while this approach has been found to work in the past for other HST cameras and in other situations, it was the least successful alternative in this case. As expected, the fit residuals at the center of the galaxy are the smallest when we allow multiple TinyTim models to account for the central AGN PSF. Furthermore, TinyTim has the advantage of producing PSF models with infinite $\mathrm{S} / \mathrm{N}$, therefore avoiding the problem of introducing additional noise into the "AGN-free" images from which we determine the host-galaxy starlight contribution. We find the same results when we also fit the image of the bright star in the field of Mrk 290 with multiple TinyTim PSF models - the residuals in the fit are decreased without adding extra noise.

Below, we provide some notes on each of the individual galaxies modeled in this work.

Mrk 142. Mrk 142 is a late-type spiral galaxy at intermediate inclination. It does not appear to have a bulge, but does seem to have a bar that manifests itself as a compact structure with a low Sérsic index $(n<1)$ and elongated shape. At the highest redshift of any of the galaxies modeled here $(z=0.045)$, the bulge may be too compact to disentangle from the very bright unresolved AGN. Its morphological classification according to these images is SBcd-SBd. The parameters for the optimal and simple surface brightness decompositions of Mrk 142 are tabulated in Table 3, and the models and residuals are displayed in Figure 2.

$S B S 1116+583 A$. SBS $1116+583 \mathrm{~A}$ is a relatively face-on barred spiral galaxy with an exponential bulge, approximately $\mathrm{SBb}$ in type. The best-fit parameters for its surface brightness decompositions, which are given in Table 4, include a lens (uniform disk; de Vaucouleurs 1959) that is more extended than the bulge, nearly circular, and has a very low Sérsic index $(n \approx 0.3-0.4)$ in both the "optimal" and "simple" models. The models and residuals are displayed in Figure 3.

Arp 151. Arp 151 (Mrk 40) is an early-type spiral galaxy (S0-Sa) with a hint of remaining spiral structure and a long tidal tail stretching north-northwest from a recent encounter with a small companion galaxy at a projected angular distance of $\sim 19^{\prime \prime}$. The messy morphology of Arp 151 and its companion required multiple surface brightness components for an accurate fit, and we do not attempt an interpretation of their physical meaning here. The best-fit parameters for its surface brightness decompositions are listed in Table 5, and the models and residuals are displayed in Figure 4.

Mrk 1310. Mrk 1310 is a ringed spiral galaxy, approximately $\mathrm{Sb}$ in type, with an apparently large number of bright globular clusters. There is also a faint galaxy directly south of Mrk 1310 that appears as an arc. The location of the galaxy along the line of sight to Mrk 1310 is unknown, but the distorted shape of this faint galaxy may mean that it is being tidally disrupted by Mrk 1310, or it may simply be a chance superposition. The large angular separation and orientation of elongation rule 
Table 5

Surface Brightness Decomposition-Arp 151

\begin{tabular}{|c|c|c|c|c|c|c|c|c|c|c|}
\hline Fit & No. & \multirow{2}{*}{$\begin{array}{l}\text { PSF+sky } \\
\text { sersic } \\
\text { fourier } \\
\text { (3) }\end{array}$} & \multirow{2}{*}{$\begin{array}{c}\Delta x\left(^{\prime \prime}\right) \\
\Delta x\left({ }^{\prime \prime}\right) \\
\quad \cdots \\
(4)\end{array}$} & $\begin{array}{l}\Delta y\left(^{\prime \prime}\right) \\
\Delta y\left(^{\prime \prime}\right) \\
\text { mode: } a_{\mathrm{n}}\end{array}$ & $\begin{array}{l}m_{\text {stmag }}{ }^{\mathrm{a}} \\
m_{\text {stmag }} \\
\phi(\operatorname{deg})\end{array}$ & \multicolumn{2}{|c|}{$\begin{array}{cc}\ldots & \text { Sky (counts) } \\
r_{\mathrm{e}}(") & n \\
\text { mode: } & a_{\mathrm{m}}, \phi(\mathrm{deg})\end{array}$} & \multicolumn{2}{|c|}{$\begin{array}{cc}\frac{d \text { sky }}{d x}\left(10^{-4} \text { counts }\right) & \frac{d \text { sky }}{d y}\left(10^{-4} \text { counts }\right) \\
b / a & \text { P.A. }(\mathrm{deg}) \\
\text { mode: } a_{\mathrm{m}}, \phi(\mathrm{deg})\end{array}$} & \multirow{2}{*}{$\begin{array}{l}\text { Note } \\
\text { (11) }\end{array}$} \\
\hline (1) & (2) & & & (5) & (6) & (7) & (8) & (9) & $(10)$ & \\
\hline \multirow[t]{21}{*}{ Optimal } & 1,2 & PSF+sky & 0 & 0 & 17.25 & $\cdots$ & 24.3 & 6.0 & -3.2 & \\
\hline & 3 & PSF & 0.00 & 0.04 & 17.55 & & & & & \\
\hline & 4 & sersic & -0.10 & -0.04 & 16.86 & 1.40 & 4.7 & 0.47 & -40.7 & \\
\hline & & fourier & $\ldots$ & $1:-0.51$ & 57.4 & 3: 0.16 & 27.7 & 4: 0.065 & -11.3 & \\
\hline & & fourier & $\ldots$ & $5:-0.024$ & 15.4 & & & & & \\
\hline & 5 & sersic & 0.09 & -0.13 & 15.99 & 2.87 & 2.5 & 0.52 & -60.7 & \\
\hline & & fourier & $\ldots$ & 1: 0.10 & 50.1 & 3: 0.10 & -34.7 & 4: 0.025 & -10.0 & \\
\hline & & fourier & $\ldots$ & $5:-0.018$ & 31.3 & & & & & \\
\hline & 6 & sersic & 1.72 & 0.62 & 16.68 & 4.59 & [1.0] & 0.22 & -54.4 & \\
\hline & & fourier & $\ldots$ & 1: 0.47 & -24.3 & $3:-0.22$ & -55.7 & 4: -0.093 & -10.1 & \\
\hline & & fourier & $\ldots$ & 5: 0.064 & -29.5 & & & & & \\
\hline & 7 & PSF & -15.60 & -10.90 & 21.30 & & & & & \\
\hline & 8 & sersic & -15.61 & -10.89 & 18.03 & 0.65 & 2.4 & 0.81 & -55.7 & \\
\hline & & fourier & $\ldots$ & $1:-0.051$ & -50.3 & $3:-0.020$ & 25.4 & & & \\
\hline & 9 & sersic & -33.17 & -20.90 & 16.01 & 5.01 & [1.0] & 0.53 & -62.0 & \\
\hline & & fourier & $\ldots$ & 1: 0.51 & -174.9 & $3:-0.14$ & -3.4 & & & \\
\hline & 10 & sersic & -33.39 & -20.85 & 21.38 & 0.29 & {$[1.0]$} & 0.27 & 24.5 & \\
\hline & & fourier & $\ldots$ & $1:-0.089$ & 29.3 & 3: 0.038 & 13.5 & & & \\
\hline & 11 & sersic & -44.54 & -2.88 & 19.67 & 1.07 & {$[1.0]$} & 0.28 & 14.8 & \\
\hline & & fourier & $\ldots$ & & -81.1 & 3: 0.061 & 45.4 & & & \\
\hline & & merit & \multicolumn{3}{|c|}{$\chi^{2}=22531868.0$} & \multicolumn{2}{|c|}{$N_{\text {dof }}=10751098$} & $N_{\text {free }}=101$ & $\chi_{v}^{2}=2.096$ & \\
\hline \multirow[t]{15}{*}{ Simple } & 1,2 & PSF+sky & 0 & 0 & 17.72 & $\ldots$ & [25.0] & 5.7 & -2.5 & \\
\hline & 3 & PSF & -0.00 & -0.03 & 17.28 & & & & & \\
\hline & 4 & PSF & -0.04 & -0.03 & 19.22 & & & & & \\
\hline & 6 & sersic & -0.11 & -0.04 & 16.55 & 1.31 & 3.4 & 0.84 & -44.2 & bulge \\
\hline & 5 & sersic & 0.14 & -0.14 & 16.20 & 4.33 & 2.7 & 0.50 & -54.8 & bulge \\
\hline & 7 & sersic & -0.21 & 0.12 & 17.34 & 5.03 & {$[1.0]$} & 0.27 & -52.4 & disk/debris \\
\hline & 8 & sersic & -15.08 & -10.71 & 16.69 & 13.87 & [1.0] & 0.17 & -56.6 & debris \\
\hline & 9 & PSF & -15.61 & -10.93 & 21.19 & & & & & \\
\hline & 10 & sersic & -15.61 & -10.93 & 18.26 & 0.50 & 1.9 & 0.87 & -55.0 & \\
\hline & 11 & sersic & -23.94 & -16.20 & 17.21 & 11.79 & [1.0] & 0.70 & 78.3 & \\
\hline & 12 & sersic & -31.16 & -19.91 & 17.31 & 7.23 & [1.0] & 0.36 & -61.5 & \\
\hline & 13 & PSF & -33.40 & -20.88 & 23.97 & & & & & \\
\hline & 14 & sersic & -33.43 & -20.84 & 18.65 & 9.73 & 4.3 & 0.32 & 26.2 & \\
\hline & 15 & sersic & -44.52 & -2.88 & 19.77 & 1.72 & {$[1.0]$} & 0.27 & 14.8 & \\
\hline & & merit & & $=2259910$ & & $N_{\text {dof }}=$ & 10751120 & $N_{\text {free }}=75$ & $\chi_{v}^{2}=2.102$ & \\
\hline
\end{tabular}

Notes. Values in square brackets were held fixed during the surface brightness model fitting.

${ }^{a}$ The STmag magnitude system is based on the absolute physical flux per unit wavelength.

out the possibility of gravitational lensing. Additional color information, at the minimum, will be necessary to determine where this small galaxy exists along our line of sight to Mrk 1310. The best-fit parameters for the surface brightness decompositions of Mrk 1310 are given in Table 6, and the models and residuals are displayed in Figure 5.

Mrk 202. Mrk 202 is a compact face-on spiral galaxy, approximately $\mathrm{Sb}$ in type, with a bright star-forming ring. The best-fit parameters for its surface brightness decompositions are listed in Table 7, and the models and residuals are displayed in Figure 6.

NGC 4253. NGC 4253 (Mrk 766) is a barred spiral galaxy of type $\mathrm{SBc}$ with a distinct nuclear spiral and a faint outer ring. It is also classified as a narrow-line Seyfert 1 because of its relatively narrow broad emission lines. The best-fit parameters for its surface brightness decompositions are given in Table 8, and the models and residuals are displayed in Figure 7.

$N G C 4748$. NGC 4748 is a barred spiral galaxy with a nuclear starbursting ring and is currently undergoing an interaction with another, slightly smaller, spiral galaxy. The best-fit parameters for its surface brightness decompositions are given in Table 9, and the models and residuals are displayed in Figure 8.

Mrk 290. Mrk 290 is an early-type spiral galaxy (Sa-Sab) at a relatively low inclination to our line of sight. The bright source to the southeast appears to be a star in our own Galaxy. The best-fit parameters for its surface brightness decompositions are listed in Table 10, and the models and residuals are displayed in Figure 9.

$N G C$ 6814. NGC 6814 is a beautiful, face-on, moderately barred spiral galaxy at a fairly low redshift of $0.0052\left(D_{L} \approx\right.$ $20 \mathrm{Mpc}$ ), making it one of the nearest broad-lined AGNs in the local universe and in our sample. The best-fit parameters for its surface brightness decompositions are given in Table 11, and the models and residuals are displayed in Figure 10.

\section{AGN FLUXES}

\subsection{Starlight Measurements and AGN Flux Recovery}

The host-galaxy starlight contribution to the $5100 \AA$ spectroscopic flux was determined by first measuring the yield of 
Table 6

Surface Brightness Decomposition-Mrk 1310

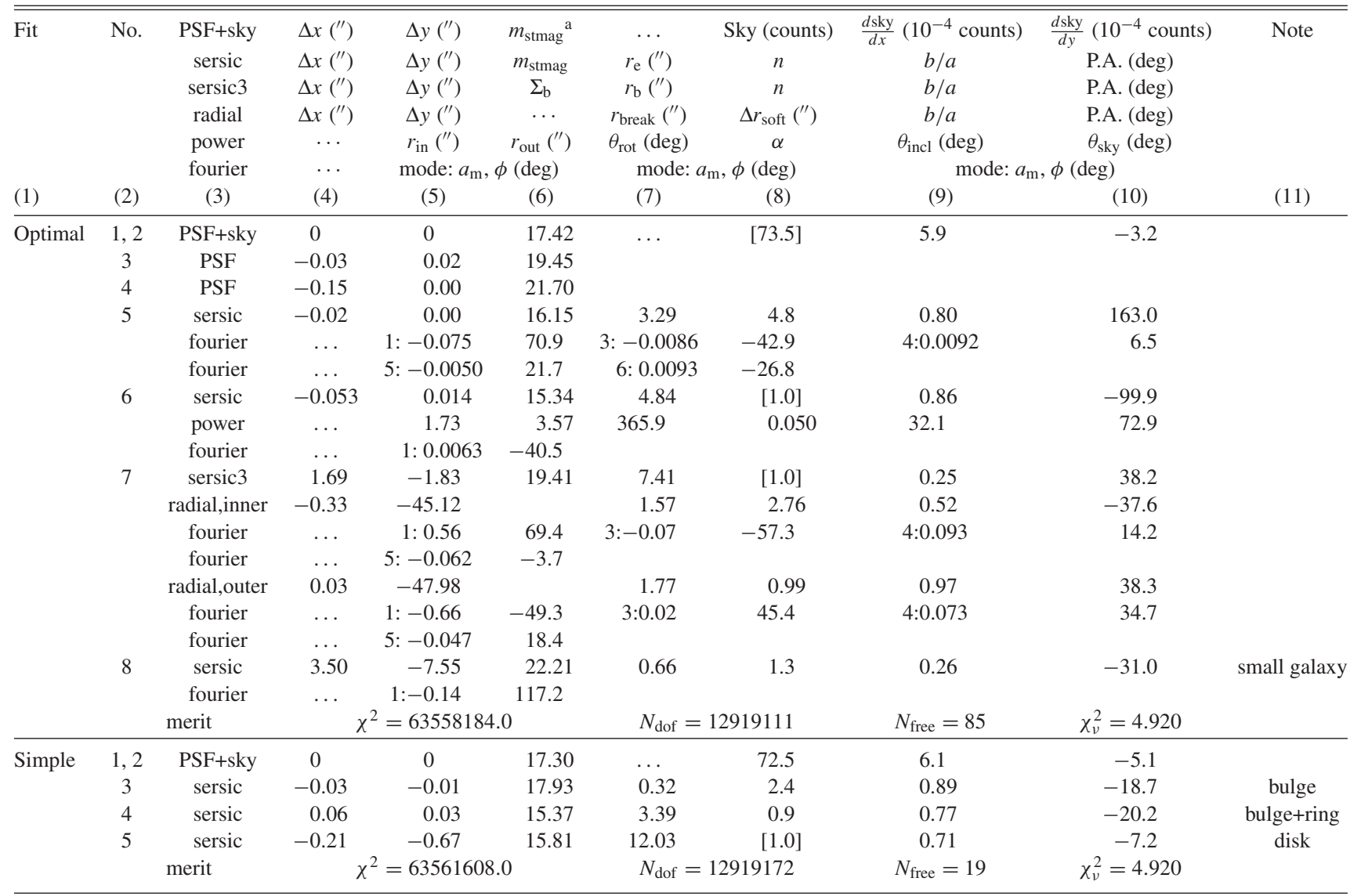

Notes. Values in square brackets were held fixed during the surface brightness model fitting.

a The STmag magnitude system is based on the absolute physical flux per unit wavelength.

electrons within a rectangular aperture, with dimensions and orientation matching that of the ground-based monitoring campaign, centered on the nucleus of the galaxy in the PSF- and sky-subtracted HST image. The exposure time and inverse sensitivity for each image (HST keyword photflam, having units of erg cm $\mathrm{cm}^{-2} \AA^{-1}$ electron $^{-1}$ ) were utilized to recover the incident photon flux from the yield of electrons. All photflam values were taken from the most recent recalibration of the appropriate data set through the HST pipeline as of 2012 June 12. For the ACS images, the photflam values are somewhat different from those previously used by Bentz et al. (2009a) because they have been updated to account for the loss of sensitivity of the High Resolution Channel over time (Bohlin et al. 2011).

Once the host-galaxy flux through the HST system had been determined, a small color correction was necessary to account for the difference between rest-frame $5100 \AA$ and the pivot wavelength ${ }^{18}$ of the filter. To determine the color correction, a bulge template spectrum (Kinney et al. 1996) was redshifted and reddened by the appropriate amounts to approximate the central host galaxy of each AGN. Only Galactic extinction was included in the reddening, and the values used are slightly different from previous values employed by Bentz et al. (2009a) because they are based on the Schlafly \& Finkbeiner (2011) recalibration of the Schlegel et al. (1998) dust map. The Galactic extinction values are smaller by a few hundredths of a magnitude for

\footnotetext{
18 A measure of the effective wavelength of a filter that is independent of the
} source spectral energy distribution (Tokunaga \& Vacca 2005). all of our sample (median difference of $-0.024 \mathrm{mag}$ ) except for $3 \mathrm{C} 120$ where the new extinction value is 0.2 mag smaller than before. The ratio of the $5100 \AA$ flux to the flux through the HST filter (which we associate with the pivot wavelength) was estimated from the redshifted and reddened spectrum using synphot, and is listed in Table 12. The final derived host-galaxy flux contributions to the ground-based spectroscopic continuum flux are given in Table 12. These values were subtracted from the absolute calibrations of the mean continuum fluxes during the monitoring campaigns to recover the mean AGN fluxes at rest-frame $5100 \AA$, from which the AGN luminosities were determined. We discuss the effects of ground-based seeing and modeling uncertainties, among others, below.

\subsection{Uncertainties}

The uncertainty in the recovered AGN flux is a combination of the mean measurement uncertainty in the continuum flux from the reverberation campaign and the uncertainty in the host-galaxy contribution to the continuum flux. The former is a small component, ranging from $1 \%$ to $5 \%$ for the measurements included here. This is due to the requirement for reverberationmapping campaigns to achieve a high $\mathrm{S} / \mathrm{N}$ per pixel in the continuum flux of each individual spectrum acquired throughout the campaign (typically $\mathrm{S} / \mathrm{N} \approx 30-100$ ) in order to measure the few-percent variations that evidence the reverberation signal. The latter contribution to the AGN flux uncertainty was determined by adding in quadrature the uncertainty in the starlight 
Table 7

Surface Brightness Decomposition-Mrk 202

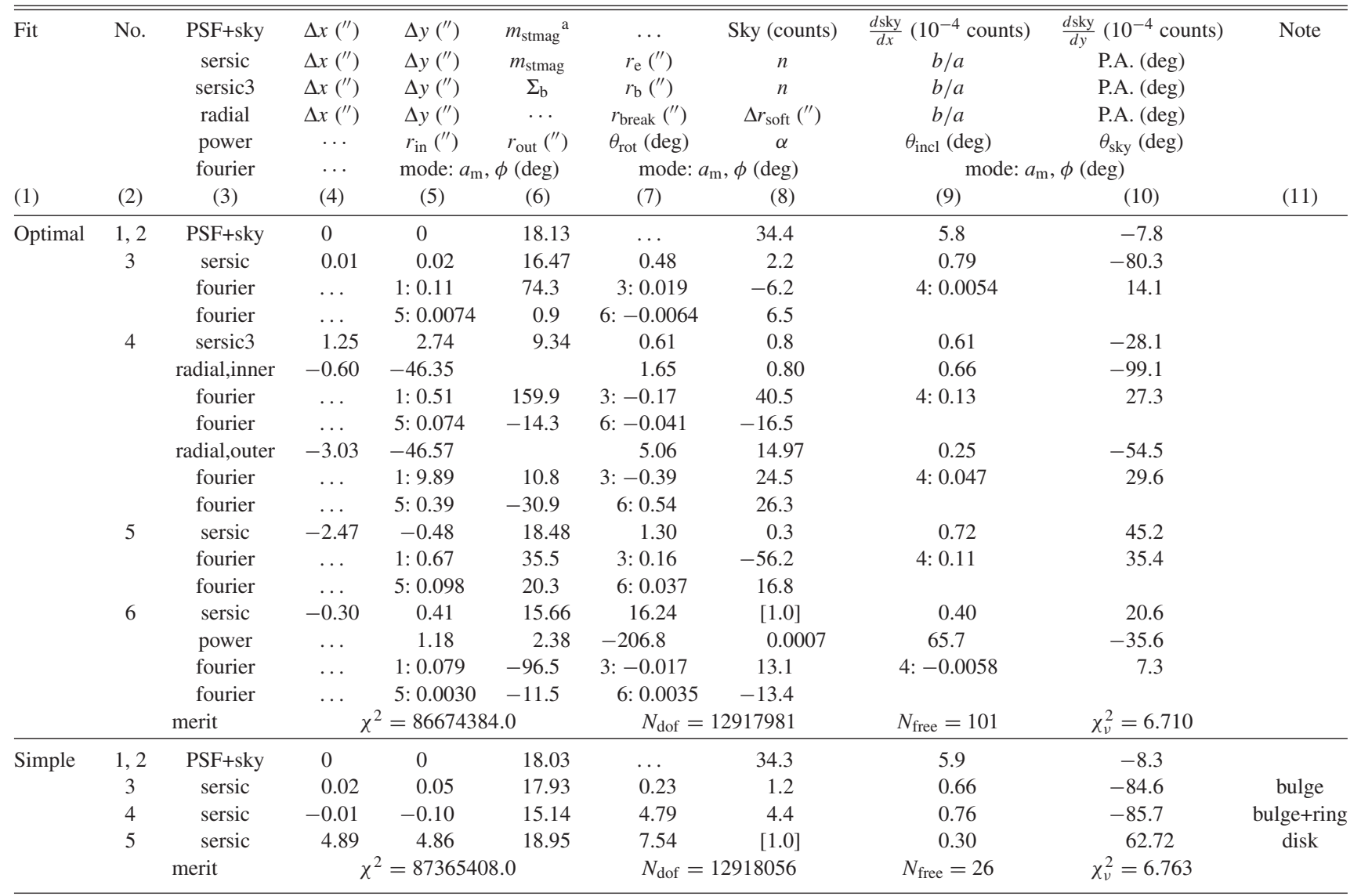

Notes. Values in square brackets were held fixed during the surface brightness model fitting.

a The STmag magnitude system is based on the absolute physical flux per unit wavelength.

flux from the modeling and the uncertainty in the starlight flux from ground-based seeing effects that would be in place during a reverberation-mapping campaign.

Modeling uncertainties. The uncertainty from surface brightness modeling was determined by comparing the starlight measurement derived for each object from the simple fit and the best fit detailed in the previous section. The addition of Fourier modes and power-law rotation, in general, changed the starlight measurement by $0.04 \%$ (Mrk 202) to $8 \%$ (Mrk 766), with a median difference of $3 \%$. Because such comparisons are timeintensive and computationally demanding, we have not carried them out for all 41 galaxies in the sample. Instead, we adopt a conservative estimate of 5\% uncertainty for the host-galaxy contribution for all compact galaxies, where the field of view of the HST image contains a large fraction of pixels that consist of empty sky (e.g., the Markarian objects and the PG quasars). For extended galaxies that fill the field of view of the HST camera with which they were observed (e.g., the NGC galaxies with the ACS HRC), we adopt a $10 \%$ uncertainty in the host-galaxy contribution due to the greater uncertainty in the determination of the background sky level during the modeling process.

Seeing effects. Optical reverberation-mapping campaigns are generally carried out from the ground and thus have to contend with variable seeing from night to night throughout a campaign. The effects of slit losses and variable seeing on the measured AGN flux are minimized by using a wide spectroscopic slit $\left(4^{\prime \prime}-5^{\prime \prime}\right)$ and by carrying out an internal calibration of all the spectra obtained for an object by utilizing the non-variable [O III] $\lambda \lambda 4959,5007$ doublet. Nevertheless, seeing redistributes the galaxy flux as well and can cause the starlight measurements from diffraction-limited HST images to differ from the contribution obtained through the ground-based setup under typical seeing conditions. Reverberation campaigns generally scale the final spectra to the [O III] flux measured on photometric nights, with a typical seeing of $\sim 1^{\prime \prime}$. To investigate the effect of seeing on the derived host-galaxy flux, we took the "AGN-free" images of NGC 5548 (an extended galaxy) and SBS 1116+583A (a compact galaxy) and created a simulated ground-based image of each by smearing with a $1^{\prime \prime}$ FWHM Gaussian. The starlight measurements were then made in the same way from the simulated ground-based images as they were from the diffraction-limited images. The difference in measured starlight flux was negligible for NGC 5548, only $2 \%$, but was $8 \%$ for SBS $1116+583$ A. Based on these results, we adopt an average $5 \%$ uncertainty for the host-galaxy contribution for each object in the sample due to ground-based seeing effects.

Background determination. Finally, we have also considered the effect of background subtraction during the spectral reductions on the host-galaxy flux. For the extended galaxy NGC 5548, we measured the host-galaxy flux in the "background" regions on either side of the extraction region. The average of these background regions was treated as "sky" flux and subtracted from the flux within the extraction region. The difference in host-galaxy flux was found to be only $2 \%$ even 
Table 8

Surface Brightness Decomposition-NGC 4253

\begin{tabular}{|c|c|c|c|c|c|c|c|c|c|c|}
\hline Fit & No. & $\begin{array}{l}\text { PSF+sky } \\
\text { sersic } \\
\text { sersic3 } \\
\text { power } \\
\text { radial } \\
\text { fourier }\end{array}$ & $\begin{array}{c}\Delta x\left(^{\prime \prime}\right) \\
\Delta x\left({ }^{\prime \prime}\right) \\
\Delta x\left(^{\prime \prime}\right) \\
\cdots \\
\Delta x\left(^{\prime \prime}\right) \\
\cdots\end{array}$ & $\begin{array}{l}\Delta y\left({ }^{\prime \prime}\right) \\
\Delta y\left({ }^{\prime \prime}\right) \\
\Delta y\left({ }^{\prime \prime}\right) \\
r_{\text {in }}\left({ }^{\prime \prime}\right) \\
\Delta y\left(^{\prime \prime}\right) \\
\text { mode: } a\end{array}$ & $\begin{array}{c}m_{\text {stmag }}{ }^{\mathrm{a}} \\
m_{\text {stmag }} \\
\Sigma_{\mathrm{b}} \\
r_{\text {out }}\left({ }^{\prime \prime}\right) \\
\cdots \\
\phi(\operatorname{deg})\end{array}$ & $\begin{array}{c}\cdots \\
r_{\mathrm{e}}\left({ }^{\prime \prime}\right) \\
r_{\mathrm{b}}\left({ }^{\prime \prime}\right) \\
\theta_{\text {rot }}(\mathrm{deg}) \\
r_{\text {break }}\left({ }^{\prime \prime}\right) \\
\text { mode: }\end{array}$ & $\begin{array}{c}\text { Sky (counts) } \\
n \\
n \\
\alpha \\
\Delta r_{\text {soft }}\left({ }^{\prime \prime}\right) \\
\text { m, } \phi(\operatorname{deg})\end{array}$ & $\begin{array}{c}\frac{d \text { sky }}{d x}\left(10^{-4} \text { counts }\right) \\
b / a \\
b / a \\
\theta_{\text {incl }}(\mathrm{deg}) \\
b / a \\
\text { mode: }\end{array}$ & $\begin{array}{c}\frac{d \text { sky }}{d y}\left(10^{-4} \text { counts }\right) \\
\text { P.A. (deg) } \\
\text { P.A. (deg) } \\
\theta_{\text {sky }(\text { deg })} \\
\text { P.A. (deg) } \\
\phi(\text { deg) }\end{array}$ & 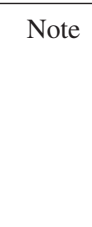 \\
\hline (1) & (2) & (3) & (4) & (5) & (6) & (7) & (8) & $(9)$ & (10) & (11) \\
\hline Optimal & $\begin{array}{c}1,2 \\
3\end{array}$ & $\begin{array}{c}\text { PSF+sky } \\
\text { sersic } \\
\text { fourier } \\
\text { sersic } \\
\text { fourier } \\
\text { fourier } \\
\text { sersic } \\
\text { power } \\
\text { fourier } \\
\text { sersic } \\
\text { sersic3 } \\
\text { radial,inner } \\
\text { fourier } \\
\text { radial,outer } \\
\text { fourier } \\
\text { merit }\end{array}$ & $\begin{array}{c}0 \\
-0.12 \\
\ldots \\
-3.73 \\
\ldots \\
\ldots \\
-0.04 \\
\ldots \\
\ldots \\
-0.01 \\
2.52 \\
-0.05 \\
\ldots \\
6.24 \\
\ldots \\
\end{array}$ & $\begin{array}{c}0 \\
0.01 \\
1:-0.25 \\
-3.76 \\
1: 0.34 \\
5: 0.073 \\
0.33 \\
{[0.00]} \\
1: 0.26 \\
0.10 \\
-14.53 \\
-45.17 \\
1: 0.11 \\
-39.60 \\
1: 0.36 \\
=18675\end{array}$ & $\begin{array}{c}15.69 \\
17.01 \\
-36.1 \\
16.82 \\
-139.3 \\
8.4 \\
15.38 \\
12.75 \\
140.8 \\
13.95 \\
21.79 \\
\ldots \\
0.0 \\
\ldots \\
-130.4 \\
1.0\end{array}$ & $\begin{array}{c}\ldots \\
0.22 \\
3: 0.25 \\
3.71 \\
3:-0.43 \\
6: 0.13 \\
6.47 \\
199.9\end{array}$ & $\begin{array}{c}{[1.0]} \\
{[1.0]} \\
17.44 \\
-6.7 \\
62.86 \\
-8.4 \\
10749147\end{array}$ & $\begin{array}{c}0.59 \\
0.72 \\
0.89 \\
4: 0.074 \\
0.39 \\
4:-0.11 \\
N_{\text {free }}=84\end{array}$ & $\begin{array}{c}-47.9 \\
11.6 \\
78.2 \\
-28.8 \\
78.9 \\
18.1 \\
\chi_{v}^{2}=1.737\end{array}$ & \\
\hline Simple & $\begin{array}{c}1,2 \\
3 \\
4 \\
5 \\
6\end{array}$ & $\begin{array}{l}\text { PSF+sky } \\
\text { sersic } \\
\text { sersic } \\
\text { sersic } \\
\text { sersic } \\
\text { merit }\end{array}$ & $\begin{array}{r}0 \\
-0.04 \\
-0.07 \\
-0.24 \\
0.78\end{array}$ & $\begin{aligned} 0 \\
0.04 \\
0.16 \\
-0.19 \\
-0.92 \\
=21660\end{aligned}$ & $\begin{array}{l}15.72 \\
17.62 \\
16.46 \\
14.68 \\
13.51\end{array}$ & $\begin{array}{r}\cdots \\
0.19 \\
1.42 \\
7.82 \\
16.17 \\
N_{\text {dof }}=\end{array}$ & $\begin{array}{c}27.6 \\
0.014 \\
1.1 \\
{[1.0]} \\
{[1.0]} \\
10749197\end{array}$ & $\begin{array}{c}3.9 \\
0.62 \\
0.56 \\
0.30 \\
0.84 \\
N_{\text {free }}=32\end{array}$ & $\begin{array}{c}-10.8 \\
-63.0 \\
-34.5 \\
-48.9 \\
-74.8 \\
\chi_{v}^{2}=2.015\end{array}$ & $\begin{array}{c}\text { nucleus } \\
\text { bulge } \\
\text { bar } \\
\text { disk }\end{array}$ \\
\hline
\end{tabular}

Notes. Values in square brackets were held fixed during the surface brightness model fitting.

a The STmag magnitude system is based on the absolute physical flux per unit wavelength.

though NGC 5548 is a bright extended galaxy. For the more compact galaxies in our sample, the effect would be even less. Therefore, we consider the effect of background-subtraction regions during spectral reductions to be negligible.

\section{DISTANCES AND AGN LUMINOSITIES}

By far, the largest contribution to the uncertainty of the AGN luminosities is from the uncertain distance to each AGN. Only five of the 41 AGNs in this study have distance measurements independent of their redshifts-NGC 3227, NGC 3783, NGC 4051, NGC 4151, and NGC 4593-while for the remaining 36 we estimate the distance from the redshift of the AGN. The distance measurements for the five aforementioned objects generally come from an average of the distance moduli for galaxies within the same group and were generated as part of a study of the "local" velocity anomaly (Tully et al. 2008); they were retrieved from the Extragalactic Distance Database (Tully et al. 2009). They are calibrated to the same zero point as the HST Key Project (Freedman et al. 2001), which found $H_{0}=72 \mathrm{~km} \mathrm{~s}^{-1} \mathrm{Mpc}^{-1}$, and which we have adopted throughout this work.

In general, we find that the uncertainties in the distances are underestimated for these individual sources in the Extragalactic Distance Database. For each of these five objects, we next give a brief discussion of the available distance measurements and their apparent quality, as well as the distances we adopt.

NGC 3227. There are seven galaxies in the same group as NGC 3227, with distance measurements ranging from 18 to $34 \mathrm{Mpc}$. Fortuitously, however, NGC 3227 is currently interacting with the early-type galaxy NGC 3226. The distance to NGC 3226 from the surface brightness fluctuation (SBF) method is $23.5 \pm 2.4 \mathrm{Mpc}$ (Tonry et al. 2001), which is $10 \%$ less than the group-averaged distance estimate to NGC 3227 of $26.4 \pm 1.6 \mathrm{Mpc}$. We adopt the distance measurement of NGC 3226 as the distance to NGC 3227.

$N G C$ 3783. The three galaxies in the group to which NGC 3783 belongs have measured distances ranging from 20 to $28 \mathrm{Mpc}$, leading to a group-averaged distance estimate of $25.1 \pm 2.9 \mathrm{Mpc}$ for NGC 3783. Based on its redshift of 0.00973, however, NGC 3783 is estimated to lie at a distance of $41 \mathrm{Mpc}$. This is a difference of nearly $50 \%$ in distance that translates into a factor of almost three difference in predicted luminosity. With a recessional velocity of $2917 \mathrm{~km} \mathrm{~s}^{-1}$, NGC 3783 would generally be expected to have peculiar velocities affecting its perceived recessional velocity at only the $\sim 10 \%$ level $\left(\sim 300 \mathrm{~km} \mathrm{~s}^{-1}\right.$, e.g., Masters et al. 2006; Bahcall \& Oh 1996), a severe underestimate given the $50 \%$ discrepancy between the group-averaged distance estimate and the estimate based on redshift. We adopt the group-averaged distance of $25.1 \mathrm{Mpc}$, with an uncertainty of $20 \%$ (5.0 Mpc), for NGC 3783.

NGC 4051. NGC 4051 is one of 64 galaxies identified as belonging to the same group. The Tully-Fisher distance to NGC 4051 is quoted as $12.2 \mathrm{Mpc}$, but it does not appear to have been corrected for the contribution of the AGN to the total galaxy luminosity. The AGN contribution would appear to make the galaxy brighter, and it would therefore seem to be nearer than it actually is. The group-averaged distance of $17.1 \pm 0.8 \mathrm{Mpc}$ includes individual galaxy distances ranging 
Table 9

Surface Brightness Decomposition—NGC 4748

\begin{tabular}{|c|c|c|c|c|c|c|c|c|c|c|}
\hline Fit & \multirow[t]{2}{*}{ No. } & \multirow[t]{2}{*}{$\begin{array}{l}\text { PSF+sky } \\
\text { sersic } \\
\text { sersic3 } \\
\text { power } \\
\text { radial } \\
\text { fourier } \\
\text { (3) }\end{array}$} & $\begin{array}{c}\Delta x\left({ }^{\prime \prime}\right) \\
\Delta x\left({ }^{\prime \prime}\right) \\
\Delta x\left({ }^{\prime \prime}\right) \\
\ldots \\
\Delta x\left(^{\prime \prime}\right) \\
\ldots\end{array}$ & $\begin{array}{l}\Delta y\left({ }^{\prime \prime}\right) \\
\Delta y\left({ }^{\prime \prime}\right) \\
\Delta y\left({ }^{\prime \prime}\right) \\
r_{\text {in }}\left({ }^{\prime \prime}\right) \\
\Delta y\left({ }^{\prime \prime}\right) \\
\text { mode: } a_{\mathrm{m}}\end{array}$ & $\begin{array}{c}m_{\text {stmag }}{ }^{\mathrm{a}} \\
m_{\mathrm{stmag}} \\
\Sigma_{\mathrm{b}} \\
r_{\text {out }}\left({ }^{\prime \prime}\right) \\
\cdots \\
\phi(\mathrm{deg})\end{array}$ & $\begin{array}{c}r_{\mathrm{e}}\left({ }^{\prime \prime}\right) \\
r_{\mathrm{b}}\left({ }^{\prime \prime}\right) \\
\theta_{\text {rot }}(\mathrm{deg}) \\
r_{\text {break }}\left({ }^{\prime \prime}\right) \\
\text { mode: }\end{array}$ & $\begin{array}{c}\text { Sky (counts) } \\
n \\
n \\
\alpha \\
\Delta r_{\text {soft }}\left({ }^{\prime \prime}\right) \\
, \phi(\text { deg })\end{array}$ & \multicolumn{2}{|c|}{$\begin{array}{cc}\frac{d \text { sky }}{d x}\left(10^{-4} \text { counts }\right) & \frac{d \text { sky }}{d y}\left(10^{-4} \text { counts }\right) \\
b / a & \text { P.A. (deg) } \\
b / a & \text { P.A. (deg) } \\
\theta_{\text {incl }}(\operatorname{deg}) & \theta_{\text {sky }}(\operatorname{deg}) \\
b / a & \text { P.A. (deg) } \\
\text { mode: } a_{\mathrm{m}}, \phi(\text { deg })\end{array}$} & \multirow[t]{2}{*}{$\begin{array}{l}\text { Note } \\
\text { (11) }\end{array}$} \\
\hline (1) & & & (4) & (5) & (6) & (7) & (8) & $(9)$ & $(10)$ & \\
\hline \multirow[t]{31}{*}{ Optimal } & 1,2 & PSF+sky & 0 & 0 & 16.58 & $\ldots$ & [38.5] & 0.0 & -0.1 & \\
\hline & \multirow[t]{3}{*}{3} & sersic & -0.02 & 0.01 & 16.17 & 0.19 & 4.8 & 0.83 & -4.7 & \\
\hline & & fourier & $\ldots$ & $1:-0.011$ & -115.2 & 3: 0.11 & 45.3 & 4: -0.024 & 2.9 & \\
\hline & & fourier & $\ldots$ & 5: 0.017 & 1.4 & $6:-0.008$ & 7.3 & & & \\
\hline & \multirow[t]{7}{*}{4} & sersic3 & -0.06 & 0.43 & 20.96 & {$[0.80]$} & {$[1.0]$} & 0.61 & -87.1 & \\
\hline & & radial,inner & 0.23 & -46.05 & $\ldots$ & 2.62 & 2.90 & 0.42 & -83.6 & \\
\hline & & fourier & $\ldots$ & 1: 0.55 & 43.1 & $3:-0.14$ & -17.0 & 4: 0.063 & 30.4 & \\
\hline & & fourier & $\ldots$ & 5: 0.038 & -25.4 & 6: 0.059 & -14.8 & & & \\
\hline & & radial,outer & 0.66 & -47.02 & $\ldots$ & 1.21 & 0.38 & 0.44 & -30.9 & \\
\hline & & fourier & $\ldots$ & 1: 0.53 & -73.6 & 3: 0.18 & 24.3 & 4: 0.072 & -22.4 & \\
\hline & & fourier & $\ldots$ & 5: 0.015 & -10.3 & 6: 0.034 & -27.7 & & & \\
\hline & \multirow[t]{3}{*}{5} & sersic & 0.26 & -0.18 & 16.28 & 2.35 & 0.5 & 0.93 & -75.7 & \\
\hline & & fourier & $\ldots$ & 1: 0.14 & 15.8 & 3: 0.021 & 52.2 & 4: 0.016 & 11.6 & \\
\hline & & fourier & $\ldots$ & 5: 0.010 & 21.3 & $6:-0.0050$ & -0.7 & & & \\
\hline & \multirow[t]{4}{*}{6} & sersic & 0.78 & 0.06 & 13.86 & 17.53 & [1.0] & 0.58 & 17.6 & \\
\hline & & power & $\ldots$ & 16.76 & 18.59 & -22.0 & 0.7 & 59.3 & 10.0 & \\
\hline & & fourier & $\ldots$ & $1:-0.13$ & 75.0 & 3: 0.064 & 10.6 & 4: 0.038 & -21.0 & \\
\hline & & fourier & $\ldots$ & 5: 0.026 & 22.5 & & & & & \\
\hline & \multirow[t]{2}{*}{7} & sersic & 11.79 & -10.61 & 17.51 & 1.12 & 0.7 & 0.78 & -83.7 & large \\
\hline & & fourier & $\ldots$ & $1:-0.28$ & 41.5 & 3: 0.029 & 20.5 & 4: -0.040 & -17.7 & companion \\
\hline & \multirow[t]{2}{*}{8} & sersic & 12.05 & -10.26 & 15.65 & 7.70 & 6.2 & 0.82 & -59.1 & \\
\hline & & fourier & $\ldots$ & $1:-0.093$ & 29.4 & 3: 0.036 & 53.2 & 4: 0.030 & -12.6 & \\
\hline & \multirow[t]{2}{*}{9} & sersic & 14.05 & -17.73 & 15.98 & 7.53 & {$[1.0]$} & 1.00 & 21.1 & \\
\hline & & fourier & $\ldots$ & $1:-0.58$ & -71.6 & 3: 0.19 & 10.7 & 4: 0.10 & 17.4 & \\
\hline & \multirow[t]{4}{*}{10} & sersic & 12.26 & -10.92 & 15.36 & 40.25 & {$[1.0]$} & 0.09 & 4.6 & \\
\hline & & power & $\ldots$ & 45.40 & 61.01 & -5.1 & 0 & 80.6 & 177.9 & \\
\hline & & fourier & $\ldots$ & 1: 0.37 & 28.2 & 3: 0.036 & -18.0 & 4: -0.034 & 6.2 & \\
\hline & & fourier & $\ldots$ & 5: 0.021 & 3.9 & & & & & \\
\hline & \multirow[t]{3}{*}{11} & sersic & 17.47 & -18.36 & 19.74 & 0.39 & 2.9 & 0.63 & 18.8 & small \\
\hline & & fourier & $\ldots$ & $1:-0.032$ & -47.8 & 3: 0.025 & -4.7 & $4:-0.012$ & 18.3 & companion \\
\hline & & merit & \multicolumn{3}{|c|}{$\chi^{2}=93724888.0$} & \multicolumn{2}{|c|}{$N_{\text {dof }}=10749060$} & $N_{\text {free }}=167$ & $\chi_{v}^{2}=8.719$ & \\
\hline \multirow[t]{10}{*}{ Simple } & 1,2 & PSF+sky & 0 & 0 & 16.75 & $\ldots$ & 38.0 & -1.7 & -2.9 & \\
\hline & 3 & sersic & -0.02 & 0.01 & 16.14 & 0.14 & 8.3 & 0.85 & -16.2 & nucleus \\
\hline & 4 & sersic & 0.36 & 0.34 & 17.36 & 0.70 & 0.1 & 0.78 & 74.2 & lens \\
\hline & 5 & sersic & 0.06 & -0.20 & 14.70 & 5.82 & 2.3 & 0.76 & 73.1 & bulge \\
\hline & 6 & sersic & -0.20 & 0.021 & 13.99 & 16.80 & {$[1.0]$} & 0.69 & -78.7 & disk \\
\hline & 7 & sersic & 12.05 & -10.25 & 18.04 & 0.50 & 3.0 & 0.76 & -48.5 & \\
\hline & 8 & sersic & 12.09 & -10.37 & 16.93 & 1.16 & 0.7 & 0.85 & -81.8 & \\
\hline & 9 & sersic & 11.61 & -9.56 & 14.97 & 7.66 & {$[1.0]$} & 0.64 & 23.7 & \\
\hline & 10 & sersic & 17.47 & -18.36 & 19.90 & 0.31 & 2.4 & 0.64 & 18.4 & \\
\hline & & merit & & $=950176$ & & $N_{\text {dof }}=$ & 0749170 & $N_{\text {free }}=51$ & $\chi_{v}^{2}=8.840$ & \\
\hline
\end{tabular}

Notes. Values in square brackets were held fixed during the surface brightness model fitting.

${ }^{a}$ The STmag magnitude system is based on the absolute physical flux per unit wavelength.

from 10 to $30 \mathrm{Mpc}$. Within the group of 64 galaxies, there is one galaxy with a Cepheid distance and eight early-type galaxies with distances from SBFs. The distances for these nine galaxies, which are expected to be more accurate on an individual basis than the distances to the other 55 galaxies in the group, span a smaller range of $10-21 \mathrm{Mpc}$, with a median value of $14.3 \mathrm{Mpc}$ that is fairly consistent with the average distance found for the full group of 64 galaxies. Based on our limited information regarding the location of NGC 4051 within its group, we adopt the group-averaged distance of $17.1 \mathrm{Mpc}$ for NGC 4051 based on all the galaxies in the same group, with an uncertainty of $3.4 \mathrm{Mpc}(20 \%)$.
NGC 4151. There are only four galaxies contributing to the group-averaged distance for NGC 4151, and their individual distances are estimated to range from $3.9 \mathrm{Mpc}$ to $34.0 \mathrm{Mpc}$ based on the Tully \& Fisher (1977) line width-luminosity correlation, with a final distance for NGC 4151 quoted as $11.2 \pm 1.1 \mathrm{Mpc}$. The object with the smallest estimated distance of $3.9 \mathrm{Mpc}$ is NGC 4151 itself, but the total galaxy luminosity does not appear to have been corrected for the enormous contribution from the AGN. It therefore appears that the distance of $3.9 \mathrm{Mpc}$ is a gross underestimate caused by neglecting the AGN contribution to the total galaxy luminosity, causing the galaxy to appear brighter (and therefore 
Table 10

Surface Brightness Decomposition-Mrk 290

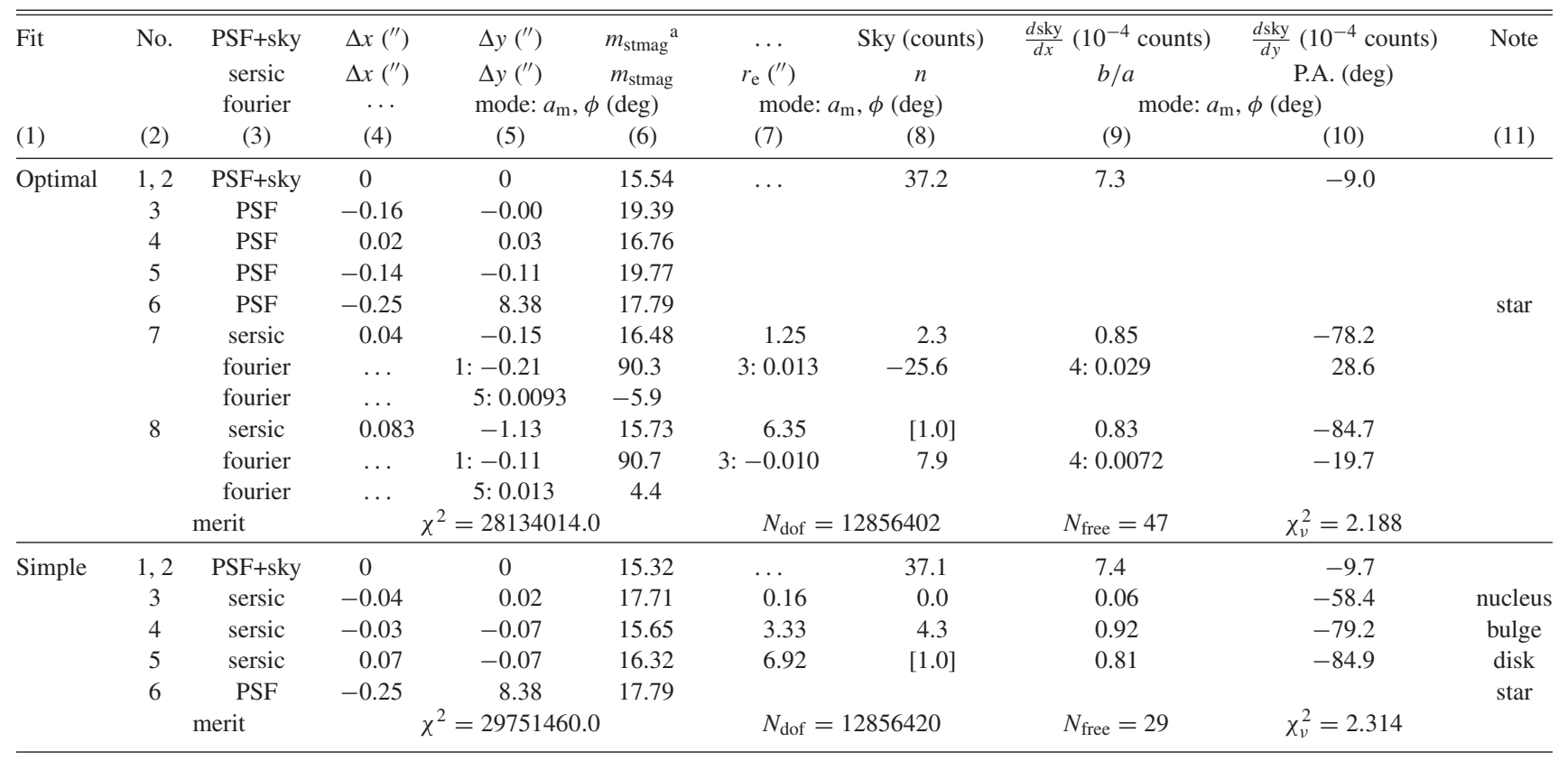

Notes. Values in square brackets were held fixed during the surface brightness model fitting.

a The STmag magnitude system is based on the absolute physical flux per unit wavelength.

Table 11

Surface Brightness Decomposition-NGC 6814

\begin{tabular}{|c|c|c|c|c|c|c|c|c|c|c|}
\hline Fit & No. & $\begin{array}{c}\text { PSF+sky } \\
\text { sersic } \\
\text { power } \\
\text { fourier }\end{array}$ & $\begin{array}{c}\Delta x\left({ }^{\prime \prime}\right) \\
\Delta x\left({ }^{\prime \prime}\right) \\
\quad \ldots \\
\quad \ldots\end{array}$ & $\begin{array}{l}\Delta y\left(^{\prime \prime}\right) \\
\Delta y\left({ }^{\prime \prime}\right) \\
r_{\text {in }}\left({ }^{\prime \prime}\right) \\
\text { mode: }\end{array}$ & $\begin{array}{c}m_{\text {stmag }}{ }^{\mathrm{a}} \\
m_{\text {stmag }} \\
r_{\text {out }}\left(^{\prime \prime}\right) \\
\phi(\text { deg })\end{array}$ & $\begin{array}{c}\cdots \\
r_{\mathrm{e}}\left({ }^{\prime \prime}\right) \\
\theta_{\text {rot }}(\mathrm{deg}) \\
\quad \text { mode: }\end{array}$ & $\begin{array}{c}\text { Sky (counts) } \\
n \\
\alpha \\
\text { n, } \phi \text { (deg) }\end{array}$ & $\begin{array}{c}\frac{d \text { sky }}{d x}\left(10^{-4} \text { counts }\right) \\
b / a \\
\theta_{\text {incl }}(\text { deg }) \\
\text { mode: } a\end{array}$ & $\begin{array}{c}\frac{d \text { sky }}{d y}\left(10^{-4} \text { counts }\right) \\
\text { P.A. (deg) } \\
\theta_{\text {sky }}(\text { deg }) \\
\phi(\text { deg })\end{array}$ & Note \\
\hline (1) & (2) & (3) & (4) & (5) & (6) & (7) & (8) & (9) & $(10)$ & (11) \\
\hline Optimal & $\begin{array}{l}1,2 \\
3 \\
4 \\
5\end{array}$ & $\begin{array}{c}\text { PSF+sky } \\
\text { PSF } \\
\text { PSF } \\
\text { sersic } \\
\text { fourier } \\
\text { sersic } \\
\text { fourier } \\
\text { sersic } \\
\text { power } \\
\text { fourier } \\
\text { sersic } \\
\text { power } \\
\text { fourier } \\
\text { nerit } \\
\end{array}$ & $\begin{array}{c}0 \\
0.01 \\
-0.07 \\
-0.17 \\
\ldots \\
-0.98 \\
\ldots \\
-7.57 \\
\ldots \\
\ldots \\
4.24 \\
\ldots \\
\ldots \\
\chi^{2} \\
\end{array}$ & \begin{tabular}{r}
\multicolumn{1}{c}{0} \\
0.06 \\
0.03 \\
0.01 \\
$1: 0.40$ \\
0.10 \\
$1: 0.21$ \\
-0.10 \\
-47.98 \\
$1: 0.44$ \\
0.07 \\
2.55 \\
$1: 0.31$ \\
$=156615$ \\
\end{tabular} & $\begin{array}{c}16.52 \\
17.99 \\
18.90 \\
15.87 \\
-74.3 \\
14.34 \\
-2.8 \\
12.80 \\
148.17 \\
15.2 \\
11.72 \\
55.83 \\
-102.6 \\
504.0\end{array}$ & $\begin{array}{c}0.90 \\
3: 0.090 \\
4.10 \\
3: 0.018 \\
28.42 \\
-386.2 \\
3: 0.15 \\
44.75 \\
-341.7 \\
3: 0.083 \\
N_{\text {dof }}=\end{array}$ & $\begin{array}{c}1.5 \\
40.3 \\
1.2 \\
1.2 \\
{[1.0]} \\
-1.038 \\
-9.4 \\
{[1.0]} \\
0.536 \\
49.0 \\
16832317 \\
\end{array}$ & $\begin{array}{c}0.71 \\
4: 0.049 \\
0.83 \\
4: 0.026 \\
0.66 \\
25.1 \\
4: 0.18 \\
0.71 \\
0.0 \\
4:-0.094 \\
N_{\text {free }}=74 \\
\end{array}$ & $\begin{array}{c}172.6 \\
15.7 \\
67.7 \\
-20.0 \\
-151.3 \\
-73.0 \\
1.7 \\
-39.1 \\
-14.5 \\
-18.6 \\
\chi_{v}^{2}=930.448 \\
\end{array}$ & \\
\hline Simple & $\begin{array}{c}1,2 \\
3 \\
4 \\
5 \\
6 \\
7 \\
8\end{array}$ & $\begin{array}{c}\text { PSF+sky } \\
\text { PSF } \\
\text { PSF } \\
\text { sersic } \\
\text { sersic } \\
\text { sersic } \\
\text { sersic } \\
\text { nerit }\end{array}$ & $\begin{array}{r}0 \\
0.01 \\
-0.08 \\
0.49 \\
-0.08 \\
-0.03 \\
-1.21 \\
\chi^{2}\end{array}$ & \begin{tabular}{r}
\multicolumn{1}{c}{0} \\
0.06 \\
0.03 \\
0.47 \\
-0.01 \\
-0.41 \\
0.98 \\
156634
\end{tabular} & $\begin{array}{r}16.53 \\
17.99 \\
18.91 \\
17.31 \\
15.06 \\
14.76 \\
11.21 \\
12.0\end{array}$ & $\begin{array}{r}1.59 \\
1.72 \\
5.87 \\
44.47 \\
N_{\text {dof }}=\end{array}$ & $\begin{array}{r}1.3 \\
2.1 \\
0.6 \\
{[1.0]} \\
16832352\end{array}$ & $\begin{array}{c}0.49 \\
0.97 \\
0.63 \\
0.98 \\
N_{\text {free }}=39\end{array}$ & $\begin{aligned} & 73.2 \\
& 21.3 \\
& 83.6 \\
& 84.7 \\
& \chi_{v}^{2}=930.556\end{aligned}$ & $\begin{array}{r}\text { bulge } \\
\text { bulge } \\
\text { bar } \\
\text { disk }\end{array}$ \\
\hline
\end{tabular}

Notes. Values in square brackets were held fixed during the surface brightness model fitting.

a The STmag magnitude system is based on the absolute physical flux per unit wavelength.

nearer) than it actually is. We have recalculated the groupaveraged distance while excluding the likely erroneous distance of $3.9 \mathrm{Mpc}$ and adopt a distance of $16.6 \pm 3.3 \mathrm{Mpc}$ for NGC 4151.
$N G C$ 4593. Only two galaxies contribute to the groupaveraged distance for NGC 4593. They have individual distance measurements of $33 \mathrm{Mpc}$ and $43 \mathrm{Mpc}$. We adopt the distance estimated by averaging their distance moduli and an uncertainty 
Table 12

Ground-based Monitoring Apertures and Measured Flux Densities

\begin{tabular}{|c|c|c|c|c|c|c|c|c|c|}
\hline \multirow{2}{*}{$\begin{array}{l}\text { Object } \\
\text { Mrk } 335\end{array}$} & \multirow{2}{*}{$\begin{array}{c}\text { Ref. }^{\text {a }} \\
1\end{array}$} & \multirow{2}{*}{$\begin{array}{c}f_{\text {obs }}[5100 \AA(1+z)] \\
\left(10^{-15} f_{\lambda}\right) \\
7.683 \pm 0.151\end{array}$} & \multicolumn{3}{|c|}{$\begin{array}{l}\text { Aperture } \\
\left("{ }^{\prime \prime}\right)\end{array}$} & \multirow{2}{*}{$\begin{array}{c}\text { P.A. } \\
\left({ }^{\circ}\right)\end{array}$} & \multirow{2}{*}{$\begin{array}{c}\begin{array}{c}\text { Photflam } \\
\left(10^{-19} \mathrm{erg} \mathrm{cm}^{-2} \AA^{-1} \mathrm{e}^{-1}\right)\end{array} \\
5.838\end{array}$} & \multirow{2}{*}{$\begin{array}{c}f_{5100} / f_{\mathrm{HST}} \\
0.847\end{array}$} & \multirow{2}{*}{$\begin{array}{c}\begin{array}{c}f_{\text {gal }}[5100 \AA(1+z)] \\
\left(10^{-15} f_{\lambda}\right)\end{array} \\
1.559 \pm 0.078\end{array}$} \\
\hline & & & 5.0 & $\times$ & 7.6 & & & & \\
\hline & 1 & $8.809 \pm 0.176$ & 5.0 & $x$ & 7.6 & 90.0 & 5.838 & 0.847 & $1.559 \pm 0.078$ \\
\hline & 2 & $7.490 \pm 0.268$ & 5.0 & $x$ & 12.0 & 0.0 & 5.838 & 0.847 & $1.646 \pm 0.082$ \\
\hline PG 0026+129 & 3 & $2.690 \pm 0.060$ & 10.0 & $x$ & 13.0 & 42.0 & 76.912 & 0.995 & $0.379 \pm 0.019$ \\
\hline PG $0052+251$ & 3 & $2.070 \pm 0.065$ & 10.0 & $x$ & 13.0 & 153.4 & 5.839 & 0.976 & $0.682 \pm 0.034$ \\
\hline Fairall 9 & 4 & $5.950 \pm 0.085$ & 4.0 & $x$ & 9.0 & 0.0 & 5.791 & 0.888 & $2.997 \pm 0.150$ \\
\hline \multirow[t]{4}{*}{ Mrk 590} & 1 & $7.895 \pm 0.170$ & 5.0 & $x$ & 7.6 & 90.0 & 5.796 & 0.848 & $3.965 \pm 0.198$ \\
\hline & 1 & $5.331 \pm 0.124$ & 5.0 & $x$ & 7.6 & 90.0 & 5.796 & 0.848 & $3.965 \pm 0.198$ \\
\hline & 1 & $6.366 \pm 0.137$ & 5.0 & $x$ & 7.6 & 90.0 & 5.796 & 0.848 & $3.965 \pm 0.198$ \\
\hline & 1 & $8.429 \pm 0.200$ & 5.0 & $x$ & 7.6 & 90.0 & 5.796 & 0.848 & $3.965 \pm 0.198$ \\
\hline \multirow[t]{2}{*}{$3 \mathrm{C} 120$} & 1 & $4.300 \pm 0.108$ & 5.0 & $x$ & 7.6 & 90.0 & 5.796 & 0.819 & $0.624 \pm 0.031$ \\
\hline & 2 & $3.370 \pm 0.084$ & 5.0 & $x$ & 12.0 & 0.0 & 5.796 & 0.819 & $0.663 \pm 0.033$ \\
\hline \multirow[t]{2}{*}{ Akn 120} & 1 & $10.365 \pm 0.220$ & 5.0 & $x$ & 7.6 & 90.0 & 5.841 & 0.846 & $5.549 \pm 0.277$ \\
\hline & 1 & $7.823 \pm 0.148$ & 5.0 & $x$ & 7.6 & 90.0 & 5.841 & 0.846 & $5.679 \pm 0.284$ \\
\hline Mrk 79 & 1 & $6.957 \pm 0.154$ & 5.0 & $x$ & 7.6 & 90.0 & 5.842 & 0.831 & $1.421 \pm 0.071$ \\
\hline & 1 & $8.487 \pm 0.156$ & 5.0 & $x$ & 7.6 & 90.0 & 5.842 & 0.831 & $1.421 \pm 0.071$ \\
\hline & 1 & $7.402 \pm 0.164$ & 5.0 & $x$ & 7.6 & 90.0 & 5.842 & 0.831 & $1.421 \pm 0.071$ \\
\hline PG 0804+761 & 3 & $5.480 \pm 0.073$ & 10.0 & $x$ & 13.0 & 315.6 & 5.840 & 0.965 & $0.664 \pm 0.033$ \\
\hline Mrk 110 & 1 & $3.454 \pm 0.074$ & 5.0 & $x$ & 7.6 & 90.0 & 5.803 & 0.869 & $0.665 \pm 0.033$ \\
\hline & 1 & $3.964 \pm 0.081$ & 5.0 & $x$ & 7.6 & 90.0 & 5.803 & 0.869 & $0.665 \pm 0.033$ \\
\hline & 1 & $2.639 \pm 0.078$ & 5.0 & $x$ & 7.6 & 90.0 & 5.803 & 0.869 & $0.665 \pm 0.033$ \\
\hline PG $0953+414$ & 3 & $1.560 \pm 0.032$ & 10.0 & $\times$ & 13.0 & 31.7 & 5.841 & 1.099 & $0.224 \pm 0.011$ \\
\hline NGC 3227 & 5 & $11.617 \pm 0.109$ & 5.0 & $x$ & $7.5^{\mathrm{a}}$ & 0.0 & 5.800 & 0.814 & $6.838 \pm 0.631$ \\
\hline Mrk 142 & 6 & $2.050 \pm 0.045$ & 4.0 & $x$ & $9.4^{\mathrm{a}}$ & 90.0 & 4.632 & 0.916 & $0.650 \pm 0.032$ \\
\hline NGC 3516 & 5 & $20.957 \pm 0.248$ & 5.0 & $\times$ & $12.0^{\mathrm{a}}$ & 0.0 & 5.828 & 0.816 & $15.36 \pm 1.54$ \\
\hline SBS $1116+583 \mathrm{~A}$ & 6 & $1.088 \pm 0.048$ & 4.0 & $x$ & $9.4^{\mathrm{a}}$ & 90.0 & 4.632 & 0.899 & $0.957 \pm 0.048$ \\
\hline Arp 151 & 6 & $1.835 \pm 0.079$ & 4.0 & $x$ & $9.4^{\mathrm{a}}$ & 90.0 & 4.632 & 0.000 & $1.241 \pm 0.062$ \\
\hline NGC 3783 & 7 & $11.380 \pm 0.604$ & 5.0 & $x$ & 10.0 & 0.0 & 5.795 & 0.801 & $4.717 \pm 0.472$ \\
\hline Mrk 1310 & 6 & $1.870 \pm 0.073$ & 4.0 & $\times$ & $9.4^{\mathrm{a}}$ & 90.0 & 4.632 & 0.000 & $1.502 \pm 0.075$ \\
\hline NGC 4051 & 5 & $13.667 \pm 0.194$ & 5.0 & $x$ & $12.0^{\mathrm{a}}$ & 0.0 & 5.799 & 0.814 & $8.738 \pm 0.874$ \\
\hline NGC 4151 & 8 & $23.800 \pm 0.539$ & 5.0 & $x$ & $12.0^{\mathrm{a}}$ & $0.0^{\mathrm{b}}$ & 5.801 & 0.811 & $17.03 \pm 1.70$ \\
\hline Mrk 202 & 6 & $1.698 \pm 0.053$ & 4.0 & $\times$ & $9.4^{\mathrm{a}}$ & 0.0 & 4.632 & 0.889 & $1.395 \pm 0.070$ \\
\hline NGC 4253 & 6 & $4.590 \pm 0.092$ & 4.0 & $x$ & $9.4^{\mathrm{a}}$ & 60.0 & 4.632 & 0.877 & $2.920 \pm 0.146$ \\
\hline PG $1226+023$ & 3 & $21.300 \pm 0.579$ & 10.0 & $\times$ & 13.0 & 171.2 & 5.845 & 0.971 & $1.303 \pm 0.065$ \\
\hline PG $1229+204$ & 3 & $2.150 \pm 0.039$ & 10.0 & $x$ & 13.0 & 291.5 & 5.842 & 0.915 & $1.326 \pm 0.066$ \\
\hline NGC 4593 & 9 & $15.853 \pm 0.319$ & 5.0 & $x$ & 12.75 & $0.0^{\mathrm{b}}$ & 5.830 & 0.820 & $7.837 \pm 0.743$ \\
\hline NGC 4748 & 6 & $4.360 \pm 0.075$ & 4.0 & $x$ & $9.4^{\mathrm{a}}$ & 0.0 & 4.632 & 0.874 & $3.207 \pm 0.160$ \\
\hline PG $1307+085$ & 3 & $1.790 \pm 0.040$ & 10.0 & $x$ & 13.0 & 186.5 & 76.912 & 0.990 & $0.230 \pm 0.012$ \\
\hline IC 4329A & 10 & $5.790 \pm 0.297$ & 5.0 & $\times$ & 10.0 & 90.0 & 5.831 & 0.824 & $3.570 \pm 0.357$ \\
\hline Mrk 279 & 11 & $6.897 \pm 0.492$ & 5.0 & $x$ & 7.5 & 90.0 & 5.796 & 0.861 & $2.918 \pm 0.146$ \\
\hline PG $1411+442$ & 3 & $3.710 \pm 0.054$ & 10.0 & $x$ & 13.0 & 347.0 & 5.842 & 0.950 & $0.769 \pm 0.038$ \\
\hline NGC 5548 & 12 & $9.916 \pm 0.494$ & 5.0 & $x$ & 7.5 & 90.0 & 5.801 & 0.833 & $3.752 \pm 0.375$ \\
\hline & 12 & $7.252 \pm 0.353$ & 5.0 & $x$ & 7.5 & 90.0 & 5.801 & 0.833 & $3.752 \pm 0.375$ \\
\hline & 12 & $9.396 \pm 0.380$ & 5.0 & $x$ & 7.5 & 90.0 & 5.801 & 0.833 & $3.752 \pm 0.375$ \\
\hline & 12 & $6.720 \pm 0.296$ & 5.0 & $x$ & 7.5 & 90.0 & 5.801 & 0.833 & $3.752 \pm 0.375$ \\
\hline & 12 & $9.062 \pm 0.351$ & 5.0 & $x$ & 7.5 & 90.0 & 5.801 & 0.833 & $3.752 \pm 0.375$ \\
\hline & 12 & $9.760 \pm 0.424$ & 5.0 & $x$ & 7.5 & 90.0 & 5.801 & 0.833 & $3.752 \pm 0.375$ \\
\hline & 12 & $12.091 \pm 0.312$ & 5.0 & $x$ & 7.5 & 90.0 & 5.801 & 0.833 & $3.752 \pm 0.375$ \\
\hline & 12 & $10.563 \pm 0.407$ & 5.0 & $x$ & 7.5 & 90.0 & 5.801 & 0.833 & $3.752 \pm 0.375$ \\
\hline & 12 & $8.120 \pm 0.306$ & 5.0 & $x$ & 7.5 & 90.0 & 5.801 & 0.833 & $3.752 \pm 0.375$ \\
\hline & 12 & $13.468 \pm 0.548$ & 5.0 & $x$ & 7.5 & 90.0 & 5.801 & 0.833 & $3.752 \pm 0.375$ \\
\hline & 12 & $11.832 \pm 0.444$ & 5.0 & $x$ & 7.5 & 90.0 & 5.801 & 0.833 & $3.752 \pm 0.375$ \\
\hline & 12 & $6.981 \pm 0.304$ & 5.0 & $x$ & 7.5 & 90.0 & 5.801 & 0.833 & $3.752 \pm 0.375$ \\
\hline & 12 & $7.032 \pm 0.334$ & 5.0 & $x$ & 7.5 & 90.0 & 5.801 & 0.833 & $3.752 \pm 0.375$ \\
\hline & 13 & $6.630 \pm 0.243$ & 5.0 & $x$ & 12.75 & $0.0^{\mathrm{b}}$ & 5.801 & 0.833 & $4.341 \pm 0.434$ \\
\hline & 6 & $6.121 \pm 0.130$ & 4.0 & $x$ & $9.4^{\mathrm{a}}$ & 60.0 & 5.801 & 0.833 & $3.537 \pm 0.354$ \\
\hline & 5 & $6.766 \pm 0.086$ & 5.0 & $x$ & $12.0^{\mathrm{a}}$ & 0.0 & 5.801 & 0.833 & $4.273 \pm 0.427$ \\
\hline PG $1426+015$ & 3 & $4.620 \pm 0.067$ & 10.0 & $\times$ & 13.0 & 341.4 & 76.912 & 0.957 & $1.134 \pm 0.105$ \\
\hline Mrk 817 & 1 & $6.098 \pm 0.120$ & 5.0 & $x$ & 7.6 & 90.0 & 5.796 & 0.864 & $1.489 \pm 0.142$ \\
\hline & 1 & $4.998 \pm 0.104$ & 5.0 & $\times$ & 7.6 & 90.0 & 5.796 & 0.864 & $1.489 \pm 0.074$ \\
\hline & 1 & $5.008 \pm 0.108$ & 5.0 & $x$ & 7.6 & 90.0 & 5.796 & 0.864 & $1.489 \pm 0.074$ \\
\hline & 5 & $6.896 \pm 0.070$ & 5.0 & $\times$ & $12.0^{\mathrm{a}}$ & 0.0 & 5.796 & 0.864 & $1.723 \pm 0.086$ \\
\hline Mrk 290 & 5 & $2.575 \pm 0.035$ & 5.0 & $x$ & $12.0^{\mathrm{a}}$ & 0.0 & 4.632 & 0.898 & $1.342 \pm 0.067$ \\
\hline PG $1613+658$ & 3 & $3.490 \pm 0.044$ & 10.0 & $x$ & 13.0 & 164.2 & 5.842 & 0.977 & $1.460 \pm 0.073$ \\
\hline PG $1617+175$ & 3 & $1.440 \pm 0.021$ & 10.0 & $x$ & 13.0 & 253.0 & 76.912 & 0.985 & $0.336 \pm 0.017$ \\
\hline
\end{tabular}


Table 12

(Continued)

\begin{tabular}{|c|c|c|c|c|c|c|c|c|c|}
\hline \multirow{2}{*}{$\begin{array}{l}\text { Object } \\
\text { PG } 1700+518\end{array}$} & \multirow{2}{*}{$\begin{array}{c}\text { Ref. }^{\mathrm{a}} \\
3\end{array}$} & \multirow{2}{*}{$\begin{array}{c}f_{\text {obs }}[5100 \AA(1+z)] \\
\left(10^{-15} f_{\lambda}\right) \\
2.200 \pm 0.011\end{array}$} & \multicolumn{3}{|c|}{$\begin{array}{l}\text { Aperture } \\
\left("{ }^{\prime \prime}\right)\end{array}$} & \multirow{2}{*}{$\begin{array}{c}\text { P.A. } \\
\left({ }^{\circ}\right)\end{array}$} & \multirow{2}{*}{$\begin{array}{c}\begin{array}{c}\text { Photflam } \\
\left(10^{-19} \mathrm{erg} \mathrm{cm}^{-2} \AA^{-1} \mathrm{e}^{-1}\right)\end{array} \\
5.842\end{array}$} & \multirow{2}{*}{$\frac{f_{5100} / f_{\mathrm{HST}}}{}$} & \multirow{2}{*}{$\begin{array}{c}f_{\mathrm{gal}}[5100 \AA(1+z)] \\
\left(10^{-15} f_{\lambda}\right) \\
0.338 \pm 0.017\end{array}$} \\
\hline & & & 10.0 & $x$ & 13.0 & & & & \\
\hline \multirow[t]{2}{*}{ 3C 390.3} & 14 & $1.732 \pm 0.188$ & 5.0 & $x$ & 7.5 & 90.0 & 5.801 & 0.899 & $0.826 \pm 0.041$ \\
\hline & 15 & $6.630 \pm 0.074$ & 3.0 & $x$ & 9.4 & 90.0 & 5.801 & 0.899 & $0.725 \pm 0.036$ \\
\hline NGC 6814 & 6 & $6.470 \pm 0.238$ & 4.0 & $x$ & $9.4^{\mathrm{a}}$ & 150.0 & 4.632 & 0.834 & $4.132 \pm 0.207$ \\
\hline Mrk 509 & 1 & $10.920 \pm 0.231$ & 5.0 & $x$ & 7.6 & 90.0 & 76.912 & 0.887 & $2.434 \pm 0.122$ \\
\hline PG 2130+099 & 2 & $3.100 \pm 0.076$ & 5.0 & $x$ & 12.0 & 0.0 & 5.794 & 0.913 & $0.574 \pm 0.029$ \\
\hline NGC 7469 & 16 & $19.023 \pm 0.572$ & 5.0 & $x$ & 12.0 & 0.0 & 5.836 & 0.822 & $8.219 \pm 0.822$ \\
\hline
\end{tabular}

Notes. Flux densities are tabulated at rest-frame $5100 \AA$ in $f_{\lambda}$ units of $\mathrm{erg} \mathrm{s}^{-1} \mathrm{~cm}^{-2} \AA^{-1}$. Position angle is defined as degrees east of north. Photflam is the inverse sensitivity of the $H S T$ detector, listed here in units of $10^{-19} \mathrm{erg} \mathrm{cm}^{-2} \AA^{-1}$ electron ${ }^{-1}$.

${ }^{a}$ The extraction width of this aperture was previously reported incorrectly. The correct extraction width is one pixel smaller and is as reported here.

$\mathrm{b}$ The position angle was erroneously reported as P.A. $=90^{\circ}$ for these monitoring campaigns previously. The actual position angle is as listed here, P.A. $=0^{\circ}$.

References. (1) Peterson et al. 1998; (2) Grier et al. 2012; (3) Kaspi et al. 2000; (4) Santos-Lleo et al. 1997; (5) Denney et al. 2009; (6) Bentz et al. 2009b; (7) Stirpe et al. 1994; (8) Bentz et al. 2006a; (9) Denney et al. 2006; (10) Winge et al. 1996; (11) Santos-Lleó et al. 2001; (12) Peterson et al. 2002 and references therein; (13) Bentz et al. 2007; (14) Dietrich et al. 1998; (15) Dietrich et al. 2012; (16) B. M. Peterson et al., in preparation.

of $20 \%(37.3 \pm 7.5 \mathrm{Mpc})$ for NGC 4593. This is consistent with the distance of $\sim 39 \mathrm{Mpc}$ expected from the redshift of NGC 4593 and assuming that NGC 4593 has zero peculiar velocity. The $20 \%$ uncertainty in the distance that we have assumed for NGC 4593 (and, indeed, several of the other distance estimates above) may be an underestimate of the true discrepancy between the actual distance to the source and our estimate of the distance.

For the other 36 AGNs in the sample, we have no choice at this time but to estimate their distances from their measured redshifts. Because of this, peculiar velocities can introduce a large uncertainty into these distance estimates. To further complicate the issue, peculiar velocities are highly direction-dependent (e.g., the "Finger of God" effect) and will be randomly oriented relative to our line of sight for the AGN host galaxies in this sample. Our lack of additional distance information for the vast majority of the AGNs in the reverberation sample leads us to conservatively estimate that peculiar velocities affect the galaxy recession velocities at an average level of $\sim 500 \mathrm{~km} \mathrm{~s}^{-1}$, or $\sim 17 \%$ for $z=0.01$. We caution that this may still be a significant underestimate of the accuracy of our assumed distances for some individual galaxies, as it would be in the above case of NGC 3783 if we had no information beyond the galaxy's redshift.

Clearly, there is a desperate need for accurate distance measurements to the AGN host galaxies in the reverberationmapping sample. The Tully-Fisher method has been shown to be accurate to $\sim 20 \%$ for individual galaxies and can reach spiral galaxies out to $z \approx 0.1$, but will require extra care for these galaxies to ensure removal of the AGN contribution to the total galaxy luminosity. Furthermore, there are a handful of galaxies in the sample that are within reach of the $\sim 30 \mathrm{Mpc}$ limit for Cepheid observations with HST. We have an approved Cycle 20 program to obtain a Cepheid-based distance measurement to the face-on spiral galaxy NGC 6814 (GO-12961, PI: Bentz). NGC 4151, in particular, is another galaxy with a very large distance uncertainty that would benefit from, and be within the reach of, an HST Cepheid program.

Finally, we note that we do not attempt to correct for internal reddening from the AGN host galaxy. Previous studies (Bentz et al. 2009a; Denney et al. 2010) have shown that such corrections, for the few objects where they are possible, are fairly small relative to the large distance uncer- tainties we have described above. In the case of the reddened AGN NGC 3227, for example, the reddening curve derived by Crenshaw et al. (2001) gives an extinction of 0.26 dex in luminosity at $5100 \AA$.

Table 13 lists the $5100 \AA$ luminosities we have determined for each of the data sets in our sample using the distances discussed above. We also give the corresponding broad $\mathrm{H} \beta$ time delays, which we take to be the average radius of the $\mathrm{H} \beta$-emitting BLR.

\section{THE RADIUS-LUMINOSITY RELATIONSHIP}

Based on previous work (Kaspi et al. 2000, 2005; Bentz et al. 2006b, 2009a), we expect the form of the $R_{\mathrm{BLR}}-L$ relationship to be a power law. We parameterize the $R_{\mathrm{BLR}}-L$ relationship here as

$$
\log \left(R_{\mathrm{BLR}} / 1 \text { lt-day }\right)=K+\alpha \log \left(\lambda L_{\lambda} / 10^{44} \mathrm{erg} \mathrm{s}^{-1}\right)
$$

To determine the best fit to the $R_{\mathrm{BLR}}-L$ relationship, we employed the LINMIX_ERR algorithm (Kelly 2007), which takes a Bayesian approach to linear regression with measurement errors in both coordinates and a component of intrinsic, random scatter. Kelly (2007) carried out extensive tests of the consistency between LINMIX_ERR and the commonly used algorithms FITEXY (Press et al. 1992) and BCES (Akritas \& Bershady 1996), finding that the best fits determined by all of the algorithms were generally consistent, but that even in cases of large scatter or poorly constrained measurements, LINMIX_ERR always derived a fit that was consistent with the known parent population from which the measurements were sampled. We fit the $R_{\mathrm{BLR}}-L$ relationship with FITEXY and BCES and found that all the algorithms provided consistent results, as we expected. We report the best-fit parameters determined by the LINMIX_ERR algorithm in Table 14.

The issue of dealing with multiple measurements for a single object when fitting the $R_{\mathrm{BLR}}-L$ relationship is not straightforward. On the one hand, if an individual AGN moves along its own $R_{\mathrm{BLR}}-L$ relationship that is parallel to the $R_{\mathrm{BLR}}-L$ relationship investigated here, then there is no real difference between multiple measurements of a single object versus measurements of many different objects. In this case, all measurements should be given equal weight in the regression analysis, regardless of which AGN they "belong" to. If, on the other hand, individual 
Table 13

Rest-frame $\mathrm{H} \beta$ Time Lags and 5100 Å Luminosities

\begin{tabular}{|c|c|c|c|}
\hline Object & $\begin{array}{l}\mathrm{H} \beta \text { Time } \mathrm{Lag} \\
\text { (days) }\end{array}$ & $\begin{array}{c}f_{\mathrm{AGN}}[5100 \AA(1+z)] \\
\left(10^{-15} \mathrm{erg} \mathrm{s}^{-1} \mathrm{~cm}^{-2} \AA^{-1}\right)\end{array}$ & $\begin{array}{c}\lambda L_{\lambda, \mathrm{AGN}}(5100 \AA) \\
\left(10^{44} \mathrm{erg} \mathrm{s}^{-1}\right)\end{array}$ \\
\hline \multirow[t]{3}{*}{ Mrk 335} & $16.8_{-4.2}^{+4.8}$ & $6.12 \pm 0.19$ & $43.70 \pm 0.06$ \\
\hline & $12.5_{-5.5}^{+6.6}$ & $7.25 \pm 0.21$ & $43.78 \pm 0.05$ \\
\hline & $14.3_{-0.7}^{+0.7}$ & $5.84 \pm 0.29$ & $43.68 \pm 0.06$ \\
\hline PG $0026+129$ & $111.0_{-28.3}^{+24.1}$ & $2.31 \pm 0.07$ & $44.91 \pm 0.02$ \\
\hline PG $0052+251$ & $89.8_{-24.1}^{+24.5}$ & $1.39 \pm 0.08$ & $44.75 \pm 0.03$ \\
\hline Fairall 9 & $17.4_{-4.3}^{+3.2}$ & $2.95 \pm 0.23$ & $43.92 \pm 0.05$ \\
\hline \multirow[t]{4}{*}{ Mrk 590} & $20.7_{-2.7}^{+3.5}$ & $3.93 \pm 0.33$ & $43.53 \pm 0.07$ \\
\hline & $14.0_{-8.8}^{+8.5}$ & $1.37 \pm 0.31$ & $43.07 \pm 0.11$ \\
\hline & $29.2_{-5.0}^{+4.9}$ & $2.40 \pm 0.31$ & $43.32 \pm 0.08$ \\
\hline & $28.8_{-4.2}^{+3.6}$ & $4.46 \pm 0.34$ & $43.59 \pm 0.06$ \\
\hline \multirow[t]{2}{*}{$3 \mathrm{C} 120$} & $38.1_{-15.3}^{+21.3}$ & $3.68 \pm 0.12$ & $44.01 \pm 0.05$ \\
\hline & $25.9_{-2.3}^{+2.3}$ & $2.71 \pm 0.10$ & $43.87 \pm 0.05$ \\
\hline \multirow[t]{2}{*}{ Ark 120} & $47.1_{-12.4}^{+8.3}$ & $4.82 \pm 0.45$ & $43.92 \pm 0.06$ \\
\hline & $37.1_{-5.4}^{+4.8}$ & $2.14 \pm 0.43$ & $43.57 \pm 0.10$ \\
\hline \multirow[t]{3}{*}{ Mrk 79} & $9.0_{-7.8}^{+8.3}$ & $5.54 \pm 0.18$ & $43.57 \pm 0.07$ \\
\hline & $16.1_{-6.6}^{+6.6}$ & $7.07 \pm 0.19$ & $43.67 \pm 0.07$ \\
\hline & $16.0_{-5.8}^{+6.4}$ & $5.98 \pm 0.19$ & $43.60 \pm 0.07$ \\
\hline PG 0804+761 & $146.9_{-18.9}^{+18.8}$ & $4.82 \pm 0.09$ & $44.85 \pm 0.02$ \\
\hline \multirow[t]{3}{*}{ Mrk 110} & $24.3_{-8.3}^{+5.5}$ & $2.79 \pm 0.09$ & $43.62 \pm 0.04$ \\
\hline & $20.4_{-6.3}^{+10.5}$ & $3.30 \pm 0.09$ & $43.69 \pm 0.04$ \\
\hline & $33.3_{-100}^{+14.9}$ & $1.97 \pm 0.09$ & $43.47 \pm 0.05$ \\
\hline PG $0953+414$ & $150.1_{-22.6}^{+21.6}$ & $1.34 \pm 0.04$ & $45.13 \pm 0.01$ \\
\hline NGC 3227 & $3.75_{-0.82}^{+0.76}$ & $4.78 \pm 0.73$ & $42.24 \pm 0.11$ \\
\hline Mrk 142 & $2.74_{-0.83}^{+0.73}$ & $1.40 \pm 0.07$ & $43.54 \pm 0.04$ \\
\hline NGC 3516 & $11.68_{-1.53}^{+1.02}$ & $5.60 \pm 1.74$ & $42.73 \pm 0.21$ \\
\hline SBS $1116+583 \mathrm{~A}$ & $2.31_{-0.49}^{+0.62}$ & $0.13 \pm 0.08$ & $42.07 \pm 0.28$ \\
\hline Arp 151 & $3.99_{-0.68}^{+0.49}$ & $0.59 \pm 0.12$ & $42.48 \pm 0.11$ \\
\hline NGC 3783 & $10.20_{-2.30}^{+3.30}$ & $6.66 \pm 0.80$ & $42.55 \pm 0.18$ \\
\hline Mrk 1310 & $3.66_{-0.61}^{+0.59}$ & $0.37 \pm 0.13$ & $42.23 \pm 0.17$ \\
\hline NGC 4051 & $1.87_{-0.50}^{+0.54}$ & $4.93 \pm 1.00$ & $41.96 \pm 0.20$ \\
\hline NGC 4151 & $6.58_{-0.76}^{+1.12}$ & $6.77 \pm 1.98$ & $42.09 \pm 0.22$ \\
\hline Mrk 202 & $3.05_{-1.12}^{+1.73}$ & $0.30 \pm 0.11$ & $42.20 \pm 0.18$ \\
\hline NGC 4253 & $6.16_{-1.22}^{+1.63}$ & $1.67 \pm 0.23$ & $42.51 \pm 0.13$ \\
\hline PG 1226+032 & $306.8_{-90.9}^{+68.5}$ & $20.00 \pm 0.59$ & $45.90 \pm 0.02$ \\
\hline PG 1229+204 & $37.8_{-15.3}^{+27.6}$ & $0.82 \pm 0.10$ & $43.64 \pm 0.06$ \\
\hline NGC 4593 & $3.73_{-0.75}^{+0.75}$ & $8.02 \pm 0.90$ & $42.87 \pm 0.18$ \\
\hline NGC 4748 & $5.55_{-2.22}^{+1.62}$ & $1.15 \pm 0.24$ & $42.49 \pm 0.13$ \\
\hline PG $1307+085$ & $105.60_{-46.60}^{+36.0}$ & $1.56 \pm 0.04$ & $44.79 \pm 0.02$ \\
\hline Mrk 279 & $16.70_{-3.90}^{+3.90}$ & $3.98 \pm 0.53$ & $43.64 \pm 0.08$ \\
\hline PG $1411+442$ & $124.30_{-61.70}^{+61.0}$ & $2.94 \pm 0.08$ & $44.50 \pm 0.02$ \\
\hline \multirow[t]{13}{*}{ NGC 5548} & $19.70_{-1.50}^{+1.50}$ & $6.16 \pm 0.65$ & $43.33 \pm 0.10$ \\
\hline & $18.60_{-2.30}^{+2.10}$ & $3.50 \pm 0.55$ & $43.08 \pm 0.11$ \\
\hline & $15.90_{-2.50}^{+2.90}$ & $5.64 \pm 0.57$ & $43.29 \pm 0.10$ \\
\hline & $11.00_{-2.00}^{+1.90}$ & $2.97 \pm 0.51$ & $43.01 \pm 0.11$ \\
\hline & $13.00_{-1.40}^{+1.60}$ & $5.31 \pm 0.55$ & $43.26 \pm 0.10$ \\
\hline & $13.40_{-4.30}^{+3.80}$ & $6.01 \pm 0.60$ & $43.32 \pm 0.10$ \\
\hline & $21.70_{-2.60}^{+2.60}$ & $8.34 \pm 0.52$ & $43.46 \pm 0.09$ \\
\hline & $16.40_{-1.10}^{+1.20}$ & $6.81 \pm 0.59$ & $43.37 \pm 0.09$ \\
\hline & $17.50_{-1.60}^{+2.00}$ & $4.37 \pm 0.52$ & $43.18 \pm 0.10$ \\
\hline & $26.50_{-2.20}^{+4.30}$ & $9.72 \pm 0.69$ & $43.52 \pm 0.09$ \\
\hline & $24.80_{-3.00}^{+3.20}$ & $8.08 \pm 0.61$ & $43.44 \pm 0.09$ \\
\hline & $6.50_{-3.70}^{+5.70}$ & $3.23 \pm 0.52$ & $43.05 \pm 0.11$ \\
\hline & $14.30_{-7.30}^{+5.90}$ & $3.28 \pm 0.54$ & $43.05 \pm 0.11$ \\
\hline
\end{tabular}


Table 13

(Continued)

\begin{tabular}{lccc}
\hline \hline Object & $\begin{array}{c}\mathrm{H} \beta \text { Time Lag } \\
(\text { days })\end{array}$ & $\begin{array}{c}f_{\text {AGN }}[5100 \AA(1+z)] \\
\left(10^{-15} \mathrm{erg} \mathrm{s}^{-1} \mathrm{~cm}^{-2} \AA^{-1}\right)\end{array}$ & $\begin{array}{c}\lambda L_{\lambda, \mathrm{AGN}}(5100 \AA) \\
\left(10^{44} \mathrm{erg} \mathrm{s}^{-1}\right)\end{array}$ \\
\hline & $6.30_{-2.30}^{+2.60}$ & $2.29 \pm 0.54$ & $42.90 \pm 0.13$ \\
& $4.18_{-1.30}^{+0.86}$ & $2.58 \pm 0.42$ & $42.95 \pm 0.11$ \\
$12.40_{-3.85}^{+2.74}$ & $2.49 \pm 0.49$ & $42.93 \pm 0.12$ \\
PG 1426+015 & $95.0_{-37.1}^{+29.9}$ & $3.49 \pm 0.14$ & $44.57 \pm 0.02$ \\
Mrk 817 & $19.00_{-3.70}^{+3.90}$ & $4.61 \pm 0.20$ & $43.73 \pm 0.05$ \\
& $15.30_{-3.50}^{+3.70}$ & $3.51 \pm 0.15$ & $43.61 \pm 0.05$ \\
& $33.60_{-7.60}^{+6.50}$ & $3.52 \pm 0.15$ & $43.61 \pm 0.05$ \\
Mrk 290 & $14.04_{-3.47}^{+3.41}$ & $5.17 \pm 0.14$ & $43.78 \pm 0.05$ \\
PG 1613+658 & $8.72_{-1.02}^{+1.21}$ & $1.23 \pm 0.10$ & $43.11 \pm 0.06$ \\
PG 1617+175 & $40.10_{-15.20}^{+15.0}$ & $2.03 \pm 0.11$ & $44.71 \pm 0.03$ \\
PG 1700+518 & $71.50_{-33.70}^{+29.6}$ & $1.10 \pm 0.03$ & $44.33 \pm 0.02$ \\
3C 390.3 & $251.8_{-38.8}^{+45.9}$ & $1.86 \pm 0.03$ & $45.53 \pm 0.01$ \\
& $23.60_{-6.70}^{+6.20}$ & $0.91 \pm 0.20$ & $43.62 \pm 0.10$ \\
NGC 6814 & $46.40_{-3.20}^{+3.60}$ & $5.91 \pm 0.09$ & $44.43 \pm 0.03$ \\
Mrk 509 & $6.64_{-0.90}^{+0.87}$ & $2.34 \pm 0.38$ & $42.05 \pm 0.29$ \\
PG 2130+099 & $79.60_{-5.40}^{+6.10}$ & $8.49 \pm 0.29$ & $44.13 \pm 0.05$ \\
NGC 7469 & $9.60_{-1.20}^{+1.20}$ & $2.53 \pm 0.09$ & $44.14 \pm 0.03$ \\
\hline
\end{tabular}

Notes. References for $\mathrm{H} \beta$ time lags are in the same order as the references in Table 12.

Table 14

$\mathrm{H} \beta R_{\mathrm{BLR}}-L$ Fits

\begin{tabular}{lcccc}
\hline \hline Note & $N$ & $K$ & $\alpha$ & $\sigma^{2}$ \\
\hline All & 71 & $1.527_{-0.031}^{+0.031}$ & $0.533_{-0.033}^{+0.035}$ & $0.036_{-0.008}^{+0.009}$ \\
Clean & 70 & $1.555_{-0.024}^{+0.024}$ & $0.542_{-0.027}^{+0.027}$ & $0.018_{-0.006}^{+0.006}$ \\
Clean2 & 69 & $1.560_{-0.024}^{+0.024}$ & $0.546_{-0.027}^{+0.027}$ & $0.018_{-0.004}^{+0.006}$ \\
ExtCorr & 71 & $1.526_{-0.030}^{+0.030}$ & $0.537_{-0.034}^{+0.034}$ & $0.037_{-0.008}^{+0.010}$ \\
Clean+ExtCorr & 70 & $1.554_{-0.024}^{+0.024}$ & $0.546_{-0.028}^{+0.027}$ & $0.018_{-0.004}^{+0.006}$ \\
Clean2+ExtCorr & 69 & $1.559_{-0.024}^{+0.024}$ & $0.549_{-0.027}^{+0.028}$ & $0.018_{-0.005}^{+0.006}$ \\
\hline
\end{tabular}

Notes. The scatter is reported as $\sigma^{2}$, the variance of the measurements around the best-fit relationship. All: all individual measurements are included in the fit. Clean: the potentially erroneous time-lag measurement for Mrk 142 is excluded from the fit, and the potentially biased time lag for PG 2130+099 is replaced with $\tau=31 \pm 4$ days. Clean2: the lags for both Mrk 142 and PG 2130+099 are excluded. ExtCorr: the luminosity of NGC 3227 is corrected for 0.26 dex of internal extinction.

AGNs have individual $R_{\mathrm{BLR}}-L$ relationships that are oriented at some other slope relative to the $R_{\mathrm{BLR}}-L$ relationship for the population, then each AGN should only be allowed to contribute a single measurement to the regression analysis. This issue has been examined for the AGN NGC 5548 by Peterson et al. (2002) and Bentz et al. (2007), and both studies found that the slope of the optical $R_{\mathrm{BLR}}-L$ relationship for NGC 5548 alone is steeper than the global relationship. Preliminary work by E. Kilerci Eser et al. (in preparation) is also finding that the slope of the optical $R_{\mathrm{BLR}}-L$ relationship is steeper for an individual object with multiple measurements and that the scatter introduced into the global relationship appears to be modest, but these results are necessarily based on only the small number of targets with multiple time-lag measurements. We have also, therefore, investigated the effect of randomly choosing only a single measurement to represent each object in the sample, and the best-fit $R_{\mathrm{BLR}}-L$ relationship is consistent within the uncertainties with the best-fit relationship derived with all the measurements for every AGN included.
Figure 11 displays the current version of the $\mathrm{H} \beta R_{\mathrm{BLR}}-L$ relationship. In the top left panel, all of the individual measurements are plotted. The new measurements that have been added to the $R_{\mathrm{BLR}}-L$ relationship in this work are shown as open circles and preferentially populate the low-luminosity end of the relationship.

\subsection{Notes on Individual Objects}

Mrk 142. The narrow-line Seyfert 1 galaxy Mrk 142 appears to be a significant outlier in the $R_{\mathrm{BLR}}-L$ relationship. With an AGN luminosity of $3.5 \times 10^{43} \mathrm{erg} \mathrm{s}^{-1}$, it has a predicted $\mathrm{H} \beta$ time lag of $\sim 20$ days, compared to its observed time lag of $\sim 3$ days. Inspection of the continuum and $\mathrm{H} \beta$ light curves presented by Bentz et al. (2009b) shows a lack of strong features, such that no apparent lag can be detected by eye. Furthermore, the cross-correlation function for Mrk 142 shows the lowest significance for the objects in the LAMP sample with reported lag detections. We suggest that the reverberation experiment for Mrk 142 should be repeated in an effort to detect a more 

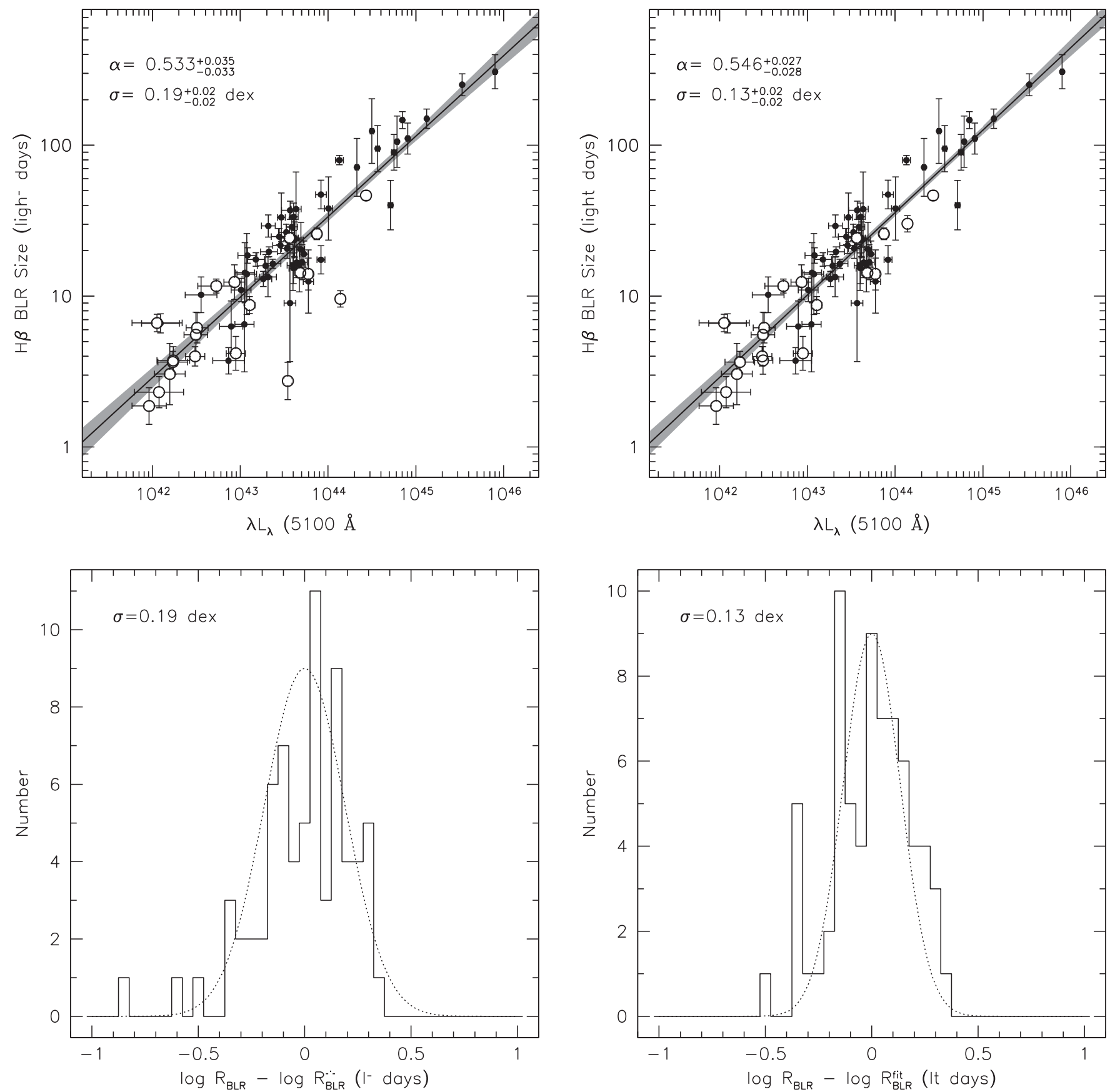

Figure 11. Top: H $\beta$ BLR radius vs. the $5100 \AA$ AGN luminosity. The solid line is the best fit to the data and the gray-scale region shows the range allowed by the uncertainties on the best fit. The left panel displays all 71 data points included in this analysis, where the open circles are the new measurements that we include for the first time. The right panel shows the fit with Mrk 142 removed, an adopted lag for PG 2130+099 of $31 \pm 4$ days, and a reddening correction of 0.26 dex for NGC 3227 (see the text for details). The slope does not change appreciably with these adjustments, but the scatter is significantly reduced from 0.19 dex to 0.13 dex. All measurements are plotted with their associated uncertainties, but the error bars are sometimes smaller than the plot symbols. Bottom: residuals of the estimated BLR radii compared to the measured BLR radii using the best fit to the $R_{\mathrm{BLR}}-L$ relationship. The dotted lines are Gaussian functions with a width equal to the variance in the scatter determined from the best fit, demonstrating the relative normality of the residual distribution.

significant time lag. Repeating the reverberation experiment will also allow for the confirmation or contradiction of the outlier status of Mrk 142. While it is possible that Mrk 142 is truly an outlier, it is worth noting that other narrow-line Seyfert 1s in the reverberation sample (NGC 4051, NGC 4253, NGC 4748) lie extremely close to their expected locations, and well within the sample scatter.

Arp 151. The tidally distorted galaxy Arp 151 was one of the most variable objects in the 2008 LAMP campaign, with a welldetermined time lag of $4.0 \pm 0.5$ days. However, there appears to be a problem with the flux calibration for Arp 151. The flux calibration for the LAMP sample of AGNs was determined by comparing the observed [O III] $\lambda 5007$ flux for NGC 5548 to the known [O III] flux from many years of spectrophotometric monitoring. Because of the unstable nature of weather and the need for many observations over a long period of time, reverberation data sets rely on the narrow [O III] emission lines as internal calibration sources for the many nonphotometric nights on which data are obtained. The emission lines do not vary on the timescales probed in a single monitoring campaign, 
and they provide the final multiplicative flux calibration factor in the spectral pipeline.

The [O III] flux of NGC 5548 is well known because of the many years that this AGN has been monitored and it was the only well-studied AGN included in the 2008 LAMP campaign. Looking at the measured [O III] fluxes for every night during the 2008 campaign, there seemed to be only a single night among the 64 nights of the campaign where the weather at Lick Observatory was steadily photometric, providing an accurate [O III] flux measurement for NGC 5548. Unfortunately, it appears that this may not have actually been the case, at least during the observations of Arp 151. The problem arises from the fact that any reasonable flux for the AGN in the HST image of Arp 151 is more than the total continuum flux of AGN+galaxy derived from the ground-based spectroscopy.

Does this mean that the process of estimating host-galaxy flux from HST imaging is flawed? Probably not. The clue comes from reviewing the final spectroscopic flux calibrations for the LAMP AGNs. The flux in the mean spectrum for each object required an increase by a multiplicative factor to match the measured [O III] flux from the single photometric night. In the case of Arp 151 (and only Arp 151), however, the [O III] flux measured from the supposed photometric night of observations is less than the mean [O III] flux from all of the observations. Reducing the mean flux by the derived multiplicative factor results in a mean continuum flux that is too small and thus produces a negative AGN flux when the host-galaxy starlight correction is applied. If we instead set the multiplicative factor equal to 1 , the problem disappears. Based on the multiplicative correction factors for all of the other objects in the LAMP sample, we should expect that this factor is $\gtrsim 1$ for Arp 151 .

Indeed, in reviewing the weather logs from the 2008 LAMP campaign and focusing only on nights logged as possibly photometric and with [O III] fluxes that are consistent with each other, we derive a flux of $0.76 \times 10^{-13} \mathrm{erg} \mathrm{s}^{-1} \mathrm{~cm}^{-2}$. This is $56 \%$ larger than the originally derived [O III] flux of $0.49 \times 10^{-13} \mathrm{erg} \mathrm{s}^{-1} \mathrm{~cm}^{-2}$. Furthermore, it is relatively consistent with the [O III] flux of $0.83 \times 10^{-13} \mathrm{erg} \mathrm{s}^{-1} \mathrm{~cm}^{-2}$ derived from potentially photometric nights during the 2011 LAMP (Barth et al. 2011) monitoring of Arp 151, given the slightly larger extraction width adopted during that campaign (10.'3 compared to the 9.'4 extraction width used in the 2008 campaign) and the overall uncertainty in the flux calibration for this single object.

We therefore adopt a flux correction factor of 1.56 as derived above, implying that the mean continuum flux density at rest-frame $5100 \AA$ for Arp 151 was $(1.835 \pm 0.079) \times$ $10^{-15} \mathrm{erg} \mathrm{s}^{-1} \mathrm{~cm}^{-2} \AA$ during the LAMP 2008 campaign. We include this corrected measurement in the values tabulated in Table 12.

$P G 2130+099$. There have been several reverberation experiments targeting PG 2130+099, yet there remains some ambiguity as to the accuracy of the reported lags. Kaspi et al. (2000) determined a time lag of $\sim 168$ days, but a reanalysis by Grier et al. (2008) found evidence for aliasing based on seasonal gaps in the light curve. Analysis of individual seasonal light curves gave much shorter time lags (16-44 days). Two new reverberation experiments have led to reported time lags of 23 days (Grier et al. 2008) and 10 days (Grier et al. 2012). The latter experiment is the most recent and has the best time sampling for this object to date: $\Delta t_{\text {med }}=0.5$ days for the continuum and $\Delta t_{\text {med }}=1.0$ days for the emission line. With a monitoring baseline of $\sim 120$ days, however, and a predicted time lag of $\sim 40$ days based on the luminosity of PG 2130+099, in retrospect the experiment is uncomfortably close to the minimum time baseline recommended for reverberation experiments (Horne et al. 2004). The most obvious feature in the continuum light curve occurs at a heliocentric Julian day of HJD-2450000 $\approx 5510$ (Grier et al. 2012, Figure 2) and would be expected to be echoed in the $\mathrm{H} \beta$ light curve at $\mathrm{HJD} \approx 5550$, right where the campaign abruptly ends. Furthermore, Grier et al. (2013) were able to reconstruct a map of the time-delay response as a function of velocity across the emission-line profile using the light curves presented by Grier et al. (2012). From this analysis, they determine that the $\mathrm{H} \beta$ time lag associated with this monitoring data set is likely to be $\sim 31$ days, not 13 days. It is therefore debatable whether PG 2130+099 is truly an outlier, and so we recommend that yet another reverberation experiment be dedicated to this object. The tightness of the $R_{\mathrm{BLR}}-L$ relationship to date implies that the discovery of a true outlier may well give important clues about detailed AGN physics deep within the potential well of the central black hole.

Because of the plausibly erroneous nature of the BLR radius measurements for Mrk 142 and PG 2130+099, we have also carried out fits to the $R_{\mathrm{BLR}}-L$ relationship with Mrk 142 excluded and with an adopted lag of $31 \pm 4$ days for PG 2130+099 (based on the analysis of Grier et al. 2013). With these two changes, the slope of the relationship is slightly increased, but still consistent within the uncertainties (see Table 14). We have also considered the fit with both Mrk 142 and PG 2130+099 excluded (see Table 14) and the fit is still consistent within the uncertainties.

Finally, we have also investigated the effect of correcting for internal extinction in the one AGN where we observe a large reddening and we have available an appropriate reddening curve, NGC 3227. As previously mentioned, work by Crenshaw et al. (2001) leads to an extinction correction of 0.26 dex at $5100 \AA$ for NGC 3227. We have applied this correction to the luminosity measurement for NGC 3227, both as a member of the full sample of 71 measurements, and with the different treatments of Mrk 142 and PG 2130+099 described above. The results are given in Table 14, and again, in both of these cases, the changes to the best-fit solution are minimal. NGC 3227 is known to be one of the most heavily reddened objects in our sample, thus, the internal extinction correction will be much smaller for the rest of the AGNs. The top right panel of Figure 11 displays the $R_{\mathrm{BLR}}-L$ relationship with Mrk 142 excluded, an adopted lag of $31 \pm 4$ days for PG 2130+099, and a reddening correction for NGC 3227.

\section{DISCUSSION}

The best-fit slope of $\alpha=0.533_{-0.033}^{+0.035}$ is consistent with the analyses previously presented by Bentz et al. (2006b, 2009a). There appears to be no difference in the relationship at the highluminosity and low-luminosity ends, with no evidence for a turnover at low luminosities. In fact, the $R_{\mathrm{BLR}}-L$ relationship appears to be remarkably consistent over four orders of magnitude in luminosity among these AGNs. Furthermore, the relationship is remarkably consistent with the expectation from simple photoionization arguments. Specifically, it was first pointed out by Davidson (1972) that we can define the ionization parameter of a BLR cloud as

$$
U=\frac{Q(H)}{4 \pi R^{2} c n_{e}},
$$


where $R$ is the distance from the central source, $c$ is the speed of light, $n_{e}$ is the electron number density, and

$$
Q(H)=\int_{v_{1}}^{\infty} \frac{L_{v}}{h v} d v
$$

is the flux of hydrogen ionizing photons emitted by the central source. Under the assumptions that the ionization parameters and particle densities are about the same for all AGNs, one finds that

$$
R \propto Q(H)^{1 / 2}
$$

so that the radius at which a particular emission line is most likely to be emitted is a simple function of the intensity of the ionizing flux. Further assuming that the ionizing continuum shape is not a function of luminosity, such that $L \propto Q(H)$, we expect

$$
R \propto L^{1 / 2} .
$$

The above arguments certainly gloss over many of the finer details of BLR photoionization physics, but this seems to matter little as the observed relationship matches this simplistic expectation quite well. This particular prediction of photoionization physics, namely that the size of the BLR should scale with the luminosity of the central source, was sought in the very early days of reverberation-mapping experiments, when the first BLR sizes were being measured (Koratkar \& Gaskell 1991). It took another decade, however, for the BLR measurements to span a sufficiently large dynamic range so that the relationship was clearly detected, despite the large initial scatter (Kaspi et al. 2000).

More recent work on the physical basis for an $R_{\mathrm{BLR}}-L$ relationship, spurred on by the initial and continuing successes of the reverberation-mapping method including near-infrared (IR) photometric reverberation mapping (Suganuma et al. 2006), has focused on the role of dust and the dust sublimation radius in setting the size of the BLR. The importance of dust was first noted by Netzer \& Laor (1993) and has been analyzed more recently by Goad et al. (2012), among others. The upshot of many of these models is that the outer edge of the BLR is bounded by the dust sublimation radius, perhaps coincident with the inner edge of the dusty torus-like structure of the unified model (Antonucci 1993). Outside the dust sublimation radius, the line emission from the dusty gas is suppressed by a large factor because the dust grains absorb many of the incoming ionizing photons as well as the emitted line photons, effectively creating an outer edge for the BLR. A natural consequence of a central ionizing source with a variable flux is that the dust sublimation radius will respond to these flux variations. An increase in ionizing photons will destroy many dust grains and increase the dust sublimation radius, whereas a decrease in ionizing flux will allow more grains to condense or migrate in and decrease the dust sublimation radius. While the basic physical motivation for an $R_{\mathrm{BLR}}-L$ relationship seems to be understood, there are many details that are currently unknown. Goad et al. (2012) provide a comprehensive overview of the state of photoionization models and the agreement (or lack, thereof) with observations.

The form of the relationship appears to be fairly well determined at this point and has not changed significantly with the updates and additions included here. The regression results are also consistent with the results of a microlensing analysis of the BLR in lensed quasars, where the magnification amplitude is dependent on the size of the emission region, an independent method that is not subject to the same uncertainties involved in reverberation mapping (Guerras et al. 2013). Furthermore, the scatter about the $R_{\mathrm{BLR}}-L$ relationship is now quite low. The LINMIX_ERR routine provides an estimate of the scatter about the relationship of $0.19 \pm 0.02 \mathrm{dex}$ (about 56\%). We plot the residuals of the measured BLR radii to the estimated BLR radii derived from the results of the LINMIX_ERR routine fit in the bottom left panel of Figure 11. The residuals are approximately normally distributed, as can be seen by comparison to an overplotted Gaussian function with $\sigma=0.19$ dex (dotted line). The scatter about the relationship is often called the "intrinsic" scatter, but in this case it is actually a combination of the real intrinsic scatter and variance from inaccurate or biased measurements. Thus, the intrinsic scatter in the $R_{\mathrm{BLR}}-L$ relationship is likely to be less than the $0.19 \pm 0.02$ dex we find here. Indeed, if we omit the two most suspicious measurements in the sample, those of Mrk 142 and PG 2130+099 as discussed above, we find that the scatter drops to 0.13 dex. The residuals with the exclusion of Mrk 142, the adopted lag of $31 \pm 4$ days for PG 2130+099, and the reddening correction for NGC 3227 are plotted in the bottom right panel of Figure 11. This is significantly lower than the typical scatter in black hole scaling relationships, such as the 0.4 dex scatter (or larger) of the $M_{\mathrm{BH}}-\sigma_{\star}$ relationship (Gültekin et al. 2009; Park et al. 2012; McConnell \& Ma 2013).

Such low scatter in the $R_{\mathrm{BLR}}-L$ relationship and the potential to decrease it even further with accurate distances and additional reverberation campaigns seem to lend support to the recent arguments of Watson et al. (2011) that the $R_{\mathrm{BLR}}-L$ relationship could potentially be used to turn any AGN with a welldetermined $\mathrm{H} \beta$ time lag into a standardizable candle for use in cosmological studies. While there is still work to be done before the $R_{\mathrm{BLR}}-L$ relationship can match the 0.05 dex scatter in the Type Ia supernova Hubble diagram residuals (e.g., Silverman et al. 2012; Ganeshalingam et al. 2013), the main weakness at the moment is the lack of accurate distance measurements to tie the current sample of reverberation-mapped AGNs onto the wellestablished nearby distance ladder. However, once this has been rectified, the radius and flux of any AGN could be measured and compared to the expected luminosity distance derived from the $R_{\mathrm{BLR}}-L$ relationship. In principle, this is possible out to $z \approx 4$ for near-IR spectroscopy of the $\mathrm{H} \beta$ emission line, far beyond the current reach of Type Ia supernovae. The high luminosities of such quasars, however, mean that the observed $\mathrm{H} \beta$ time lags would be on the order of decades. Because the BLR is ionization stratified, emissions lines with a higher ionization potential than $\mathrm{H} \beta$, such as $\mathrm{C}$ IV or He II, have rest-frame time delays that are factors of a few smaller than those of $\mathrm{H} \beta$. If separate $R_{\mathrm{BLR}}-L$ relationships for these lines could be defined and calibrated as accurately as we have achieved for $\mathrm{H} \beta$ (see Kaspi et al. 2007 for current progress on the C IV $R_{\mathrm{BLR}}-L$ relationship), then exploring cosmology with reverberation experiments would become much more feasible for objects with $z>2$, where the expected observed time delays suffer heavily from time-dilation effects. The ability to probe out to such high redshifts would provide observational constraints on the evolution of the dark energy equation-of-state parameter, as well as on alternative theories of gravity (A. L. King et al., in preparation).

\section{FUTURE WORK}

The most pressing deficiency in the $\mathrm{H} \beta R_{\mathrm{BLR}}-L$ relationship is the current lack of accurate distance measurements to the AGN host galaxies. The uncertainty in the distances at present 
provides the single largest source of uncertainty in the AGN luminosity measurements, especially as Tully et al. (2008) have shown that peculiar velocities may still be important $(>10 \%)$ even beyond $50 \mathrm{Mpc}$. We are working to obtain distances based on the Tully-Fisher method for the intermediate inclination spirals in our sample with new H I spectroscopy and near-infrared imaging. We will also obtain a Cepheid-based distance for NGC 6814 (HST GO-12961, PI: Bentz), and will explore additional distance indicators (such as the globular cluster luminosity function and the planetary nebula luminosity function) for the remaining AGN host galaxies in the sample. New reverberation campaigns should be dedicated to studying the two most likely outliers in the $R_{\mathrm{BLR}}-L$ relationship-PG 2130+099 and Mrk 142-to determine whether they are truly outliers. Additionally, the difficulties with the flux calibration of Arp 151 suggest that it would be a useful exercise to acquire new spectra of all the objects in the sample, taken under stable photometric conditions and with a uniform setup.

There is also much work to be done to determine independent $R_{\mathrm{BLR}}-L$ relationships for C IV and other emission lines for use in estimating black hole masses at higher redshifts, where the $\mathrm{H} \beta$ emission line has redshifted out of the observed optical bandpass. All of the derived $R_{\mathrm{BLR}}-L$ and black hole mass scaling relationships for all other emission lines in the literature currently rely on the $\mathrm{H} \beta R_{\mathrm{BLR}}-L$ relationship. It is therefore critical that we build up independent $R_{\mathrm{BLR}}-L$ relationships for these other commonly utilized emission lines. While $\mathrm{Mg}$ II and $\mathrm{C}$ IV are frequently employed for black hole mass estimates at $z \gtrsim 0.5$, there are only a handful of existing $\mathrm{C}$ IV reverberation results (see Kaspi et al. 2007), with the vast majority centered around a very small range in luminosities, and in the case of $\mathrm{Mg}$ II, only a single object has a measured reverberation time lag. Furthermore, the C IV time lags are generally deduced from International Ultraviolet Explorer spectra that were obtained every few days, and so have relatively poor temporal sampling compared to what is typically achieved for $\mathrm{H} \beta$ (Peterson et al. 2004). The higher ionization state of C IV means that we expect its time lag to be a factor of 2-3 shorter than that of $\mathrm{H} \beta$. Future $\mathrm{C}$ IV reverberation experiments of low- to moderate-luminosity AGNs in the nearby universe will require daily sampling or better in order to measure C IV time delays to the same level of significance that is now typically achieved for $\mathrm{H} \beta$.

Finally, although this empirical relationship is well measured and there seems to be some theoretical understanding behind it, the details of the photoionization physics, as well as the geometry and kinematics of the gas, are not well understood at this time. There is significant room for improvement in our physical understanding of AGN BLRs.

\section{SUMMARY}

We have carried out an imaging program with HST to provide starlight corrections to the luminosities of nine AGNs with $\mathrm{H} \beta$ radius measurements. We have fully updated and revised the calibration sample for the $R_{\mathrm{BLR}}-L$ relationship, including 20 new $\mathrm{H} \beta$ BLR measurements from recent reverberationmapping campaigns, and we have reinvestigated the form of the relationship. We find a best fit of $\log \left(R_{\mathrm{BLR}} / 1 \mathrm{lt}\right.$-day $)=$ $1.527_{-0.031}^{+0.031}+0.533_{-0.033}^{+0.035} \log \left(\lambda L_{\lambda} / 10^{44} L_{\odot}\right)$. This is consistent with a slope of 0.5 and with previous work that included starlight corrections to the AGN luminosity measurements. After including the additions and updates, the single largest source of uncertainty comes from the highly uncertain distances to the AGNs in the sample. The low scatter in the relationship
$(0.19 \pm 0.02 \mathrm{dex})$ and the potential to further reduce the scatter, with no clear outliers, support the proposed use of the $R_{\mathrm{BLR}}-L$ relationship to probe the matter and energy content of the universe out to $z \approx 0.6$ with optical measurements of the $\mathrm{H} \beta$ emission line. Pushing the $\mathrm{H} \beta$ observations into the near-IR would allow the relationship to probe quasars out to $z \approx 4$, beyond the reach of Type Ia supernovae and into a new interesting regime for tests of the predictions of different cosmological models.

This work is based on observations with the NASA/ESA Hubble Space Telescope. We are grateful for support of this work through grant HST GO-11662 from the Space Telescope Science Institute, which is operated by the Association of Universities for Research in Astronomy, Inc., under NASA contract NAS5-26555. K.D.D. has received funding from the People Programme (Marie Curie Actions) of the European Union's Seventh Framework Programme FP7/2007-2013/ under REA grant agreement No. 300553. A.J.B. acknowledges support from NSF grant AST-1108835. B.M.P., C.J.G., G.D.R., and R.W.P. acknowledge support from NSF grant AST-1008882 to Ohio State University. A.V.F. is grateful for the support of NSF grants AST-1108665 and AST-1211916, the TABASGO Foundation, and the Christopher R. Redlich Fund. The work of D.S. was carried out at Jet Propulsion Laboratory, California Institute of Technology, under a contract with NASA. J.H.W. acknowledges the support by the National Research Foundation of Korea (NRF) grant funded by the Korean government (No. 2012-006087). This research has made use of the NASA/ IPAC Extragalactic Database (NED) which is operated by the Jet Propulsion Laboratory, California Institute of Technology, under contract with the National Aeronautics and Space Administration and the SIMBAD database, operated at CDS, Strasbourg, France. We dedicate this paper to the memory of our dear friend and colleague, Weidong $\mathrm{Li}$, whose tireless dedication to the Katzman Automatic Imaging Telescope (KAIT) significantly contributed to the success of LAMP; his premature, tragic passing has deeply saddened us. We thank the Dark Cosmology Center for their hospitality during the Improving Black Hole Masses in Active Galaxies workshop in July 2012, which provided a venue for conversations that improved this work.

\section{REFERENCES}

Akritas, M. G., \& Bershady, M. A. 1996, ApJ, 470, 706

Antonucci, R. 1993, ARA\&A, 31, 473

Bahcall, N. A., \& Oh, S. P. 1996, ApJL, 462, L49

Barth, A. J., Pancoast, A., Thorman, S. J., et al. 2011, ApJL, 743, L4

Bennert, V. N., Treu, T., Woo, J.-H., et al. 2010, ApJ, 708, 1507

Bentz, M. C., Denney, K. D., Cackett, E. M., et al. 2006a, ApJ, 651, 775

Bentz, M. C., Denney, K. D., Cackett, E. M., et al. 2007, ApJ, 662, 205

Bentz, M. C., Peterson, B. M., Netzer, H., Pogge, R. W., \& Vestergaard, M. 2009a, ApJ, 697, 160

Bentz, M. C., Peterson, B. M., Pogge, R. W., Vestergaard, M., \& Onken, C. A. 2006b, ApJ, 644, 133

Bentz, M. C., Walsh, J. L., Barth, A. J., et al. 2009b, ApJ, 705, 199

Bentz, M. C., Walsh, J. L., Barth, A. J., et al. 2010, ApJ, 716, 993

Blandford, R. D., \& McKee, C. F. 1982, ApJ, 255, 419

Bohlin, R. C., Mack, J., \& Ubeda, L. 2011, Flux Calibration of the ACS CCD Cameras III. Sensitivity Changes over Time, Tech. Report

Brewer, B. J., Treu, T., Pancoast, A., et al. 2011, ApJL, 733, L33

Brown, M. E., Trujillo, C., \& Rabinowitz, D. 2004, ApJ, 617, 645

Cao, X. 2010, ApJ, 724, 855

Crenshaw, D. M., Kraemer, S. B., Bruhweiler, F. C., \& Ruiz, J. R. 2001, ApJ, 555,633

Davidson, K. 1972, ApJ, 171, 213

Davies, R. I., Thomas, J., Genzel, R., et al. 2006, ApJ, 646, 754 
de Vaucouleurs, G. 1948, AnAp, 11, 247

de Vaucouleurs, G. 1959, HDP, 53, 275

Denney, K. D., Bentz, M. C., Peterson, B. M., et al. 2006, ApJ, 653, 152

Denney, K. D., Peterson, B. M., Pogge, R. W., et al. 2010, ApJ, 721, 715

Denney, K. D., Watson, L. C., Peterson, B. M., et al. 2009, ApJ, 702, 1353

Dietrich, M., Peterson, B. M., Albrecht, P., et al. 1998, ApJS, 115, 185

Dietrich, M., Peterson, B. M., Grier, C. J., et al. 2012, ApJ, 757, 53

Dong, X., Wang, T., Yuan, W., et al. 2007, ApJ, 657, 700

Drake, A. J., Djorgovski, S. G., Mahabal, A., et al. 2011, ApJ, 735, 106

Freedman, W. L., Madore, B. F., Gibson, B. K., et al. 2001, ApJ, 553, 47

Ganeshalingam, M., Li, W., \& Filippenko, A. V. 2013, MNRAS, submitted

Ghisellini, G., \& Tavecchio, F. 2010, MNRAS, 409, L79

Goad, M. R., Korista, K. T., \& Ruff, A. J. 2012, MNRAS, 426, 3086

Greene, J. E., \& Ho, L. C. 2007a, ApJ, 670, 92

Greene, J. E., \& Ho, L. C. 2007b, ApJ, 667, 131

Grier, C. J., Peterson, B. M., Bentz, M. C., et al. 2008, ApJ, 688, 837

Grier, C. J., Peterson, B. M., Horne, K., et al. 2013, ApJ, 764, 47

Grier, C. J., Peterson, B. M., Pogge, R. W., et al. 2012, ApJ, 755, 60

Guerras, E., Mediavilla, E., Jimenez-Vicente, J., et al. 2013, ApJ, 764, 160

Gültekin, K., Richstone, D. O., Gebhardt, K., et al. 2009, ApJ, 698, 198

Hicks, E. K. S., \& Malkan, M. A. 2008, ApJS, 174, 31

Horne, K., Peterson, B. M., Collier, S. J., \& Netzer, H. 2004, PASP, 116, 465

Kaspi, S., Brandt, W. N., Maoz, D., et al. 2007, ApJ, 659, 997

Kaspi, S., Maoz, D., Netzer, H., et al. 2005, ApJ, 629, 61

Kaspi, S., Smith, P. S., Netzer, H., et al. 2000, ApJ, 533, 631

Kelly, B. C. 2007, ApJ, 665, 1489

Kelly, B. C., Vestergaard, M., \& Fan, X. 2009, ApJ, 692, 1388

Kim, M., Ho, L. C., Peng, C. Y., Barth, A. J., \& Im, M. 2008, ApJS, 179, 283

Kinney, A. L., Calzetti, D., Bohlin, R. C., et al. 1996, ApJ, 467, 38

Kollatschny, W. 2003, A\&A, 407, 461

Koratkar, A. P., \& Gaskell, C. M. 1991, ApJL, 370, L61

Krist, J. 1993, in ASP Conf. Ser. 52, Astronomical Data Analysis Software and Systems II, ed. R. J. Hanisch, R. J. V. Brissenden, \& J. Barnes (San Francisco, CA: ASP), 536

Laor, A. 1998, ApJL, 505, L83

Masters, K. L., Springob, C. M., Haynes, M. P., \& Giovanelli, R. 2006, ApJ, 653,861

Mathur, S., Fields, D., Peterson, B. M., \& Grupe, D. 2012, ApJ, 754, 146

McConnell, N. J., \& Ma, C.-P. 2013, ApJ, 764, 184

McLure, R. J., \& Jarvis, M. J. 2002, MNRAS, 337, 109

Merloni, A., Bongiorno, A., Bolzonella, M., et al. 2010, ApJ, 708, 137

Moffat, A. F. J. 1969, A\&A, 3, 455

Mortlock, D. J., Warren, S. J., Venemans, B. P., et al. 2011, Natur, 474, 616

Netzer, H., \& Laor, A. 1993, ApJL, 404, L51

Onken, C. A., Valluri, M., Peterson, B. M., et al. 2007, ApJ, 670, 105

Pancoast, A., Brewer, B. J., \& Treu, T. 2011, ApJ, 730, 139

Pancoast, A., Brewer, B. J., Treu, T., et al. 2012, ApJ, 754, 49

Papadakis, I. E., Brinkmann, W., Gliozzi, M., \& Raeth, C. 2010, A\&A, $518, \mathrm{~A} 28$
Park, D., Kelly, B. C., Woo, J.-H., \& Treu, T. 2012, ApJS, 203, 6

Peng, C. Y., Ho, L. C., Impey, C. D., \& Rix, H. 2002, AJ, 124, 266

Peng, C. Y., Ho, L. C., Impey, C. D., \& Rix, H.-W. 2010, AJ, 139, 2097

Peterson, B. M., Berlind, P., Bertram, R., et al. 2002, ApJ, 581, 197

Peterson, B. M., Ferrarese, L., Gilbert, K. M., et al. 2004, ApJ, 613, 682

Peterson, B. M., \& Wandel, A. 1999, ApJL, 521, L95

Peterson, B. M., \& Wandel, A. 2000, ApJL, 540, L13

Peterson, B. M., Wanders, I., Bertram, R., et al. 1998, ApJ, 501, 82

Press, W. H., Teukolsky, S. A., Vetterling, W. T., \& Flannery, B. P. 1992, Numerical Recipes in FORTRAN. The Art of Scientific Computing (2nd ed.; Cambridge: Cambridge Univ. Press), 660

Sambruna, R. M., Gliozzi, M., Tavecchio, F., Maraschi, L., \& Foschini, L. 2006, ApJ, 652, 146

Santos-Lleo, M., Chatzichristou, E., Mendes de Oliveira, C., et al. 1997, ApJS, 112,271

Santos-Lleó, M., Clavel, J., Schulz, B., et al. 2001, A\&A, 369, 57

Schlafly, E. F., \& Finkbeiner, D. P. 2011, ApJ, 737, 103

Schlegel, D. J., Finkbeiner, D. P., \& Davis, M. 1998, ApJ, 500, 525

Schulze, A., \& Wisotzki, L. 2010, A\&A, 516, A87

Sérsic, J. L. 1968, Atlas de Galaxias Australes (Cordoba, Argentina: Observatorio Astronomico)

Shankar, F., Weinberg, D. H., \& Miralda-Escudé, J. 2009, ApJ, 690, 20

Silverman, J. M., Ganeshalingam, M., Li, W., \& Filippenko, A. V. 2012, MNRAS, 425, 1889

Stirpe, G. M., Winge, C., Altieri, B., et al. 1994, ApJ, 425, 609

Suganuma, M., Yoshii, Y., Kobayashi, Y., et al. 2006, ApJ, 639, 46

Tavecchio, F., Maraschi, L., Wolter, A., et al. 2007, ApJ, 662, 900

Thornton, C. E., Barth, A. J., Ho, L. C., Rutledge, R. E., \& Greene, J. E. 2008, ApJ, 686, 892

Tokunaga, A. T., \& Vacca, W. D. 2005, PASP, 117, 421

Tonry, J. L., Dressler, A., Blakeslee, J. P., et al. 2001, ApJ, 546, 681

Trump, J. R., Impey, C. D., Kelly, B. C., et al. 2011, ApJ, 733, 60

Tully, R. B., \& Fisher, J. R. 1977, A\&A, 54, 661

Tully, R. B., Rizzi, L., Shaya, E. J., et al. 2009, AJ, 138, 323

Tully, R. B., Shaya, E. J., Karachentsev, I. D., et al. 2008, ApJ, 676, 184

van Dokkum, P. G. 2001, PASP, 113, 1420

Vestergaard, M., Fan, X., Tremonti, C. A., Osmer, P. S., \& Richards, G. T. 2008, ApJL, 674, L1

Vestergaard, M., \& Osmer, P. S. 2009, ApJ, 699, 800

Vestergaard, M., \& Peterson, B. M. 2006, ApJ, 641, 689

Wandel, A., Peterson, B. M., \& Malkan, M. A. 1999, ApJ, 526, 579

Wang, J., Mao, Y. F., \& Wei, J. Y. 2009, AJ, 137, 3388

Watson, D., Denney, K. D., Vestergaard, M., \& Davis, T. M. 2011, ApJL, 740, L49

Willott, C. J., Albert, L., Arzoumanian, D., et al. 2010, AJ, 140, 546

Winge, C., Peterson, B. M., Pastoriza, M. G., \& Storchi-Bergmann, T. 1996, ApJ, 469,648

Woo, J.-H., Treu, T., Malkan, M. A., \& Blandford, R. D. 2008, ApJ, 681,925 